IMA Journal of Numerical Analysis (2016) Page 1 of 40 doi:10.1093/imanum/drnxxx

\title{
Two-grid methods for a class of nonlinear elliptic eigenvalue problems
}

\author{
ERIC CANCÈs $\dagger$ \\ Université Paris-Est, CERMICS, Ecole des Ponts and INRIA, 6 \& 8 avenue Blaise Pascal, \\ 77455 Marne-la-Vallée, France \\ AND \\ RACHIDA CHAKIR $\ddagger$ \\ Université Paris-Est, IFSTTAR, 14-20 Bd Newton, Cité Descartes, Champs-sur-Marne, 77447 \\ Marne-la-Vallée Cedex 2, France \\ AND \\ LIANHUA HE \\ Sorbonne Universits, UPMC Univ Paris 06, UMR 7598, Laboratoire Jacques-Louis Lions, \\ F-75005, Paris, France \\ AND \\ YVON MADAYII \\ Sorbonne Universités, UPMC Univ Paris 06, UMR 7598, Laboratoire Jacques-Louis Lions, \\ F-75005, Paris, France; Institut Universitaire de France and Division of Applied \\ Mathematics, Brown University, Providence, RI, USA
}

[Received on $\mathrm{xxx}$; revised on $\mathrm{xxx}$ ]

\begin{abstract}
In this paper, we introduce and analyze some two-grid methods for nonlinear elliptic eigenvalue problems of the form $-\operatorname{div}(\mathscr{D} \nabla u)+V u+f\left(u^{2}\right) u=\lambda u,\|u\|_{L^{2}}=1$. We provide $a$ priori error estimates for the ground state energy, the eigenvalue $\lambda$, and the eigenfunction $u$, in various Sobolev norms. We focus in particular on the Fourier spectral approximation (for periodic boundary conditions), and on the $\mathbb{P}_{1}$ and $\mathbb{P}_{2}$ finite element discretizations (for homogeneous Dirichlet boundary conditions), taking numerical integration errors into account. Finally, we provide numerical examples illustrating our analysis.
\end{abstract}

Keywords: Nonlinear eigenvalue problem, Spectral and pseudo spectral approximation, Finite element approximation, Ground state computation, Numerical analysis, Two-grid method.

\section{Introduction}

Nonlinear eigenvalue problems are encountered in various applications in sciences and engineering, including the simulation of Bose-Einstein condensates (Gross-Pitaevskii equation, see e.g. Pitaevskii \& Stringari (2003)), electronic structure calculation (Hartree-Fock method, orbital free and Kohn-Sham Density Functional Theory), and the study of the vibration modes of structures in nonlinear elasticity.

\footnotetext{
${ }^{\dagger}$ Email: cances@cermics.enpc.fr

†rachida.chakir@ifsttar.fr

§helianhua86@gmail.com

ICorresponding author. Email: maday@ann.jussieu.fr
}

(C) The author 2016. Published by Oxford University Press on behalf of the Institute of Mathematics and its Applications. All rights reserved. 
The first results on the numerical analysis of nonlinear eigenvalue problems have been published in Zhou (2004). These first results were improved by three of us in Cancès et al. (2010), where optimal a priori error bounds for nonlinear elliptic eigenvalue problems were obtained for the first time. The techniques introduced in Cancès et al. (2010), based on estimates in negative Sobolev norms, have then been applied to a variety of nonlinear eigenvalue problems (see Cancès et al. (2012); Chen et al. (2013)), among which the Kohn-Sham problem (Kohn \& Sham (1965)), which is currently one of the most widely used models in computational physics and chemistry.

As in Cancès et al. (2010), we focus on the nonlinear elliptic eigenvalue problems arising in the study of variational problems of the form

$$
I=\inf \left\{E(v), v \in X, \int_{\Omega} v^{2}=1\right\}
$$

where

$$
\begin{aligned}
& \Omega \text { is a regular bounded domain or a rectangular brick of } \mathbb{R}^{d} \text { and } X=H_{0}^{1}(\Omega), \\
& \text { or } \\
& \Omega \text { is the unit cell of a periodic lattice } \mathscr{R} \text { of } \mathbb{R}^{d} \text { and } X=H_{\#}^{1}(\Omega),
\end{aligned}
$$

with $d=1,2$, or $3, H_{\#}^{1}(\Omega)$ denoting the space of the restrictions to $\Omega$ of the $H_{\mathrm{loc}}^{1}, \mathscr{R}$-periodic functions on $\mathbb{R}^{d}$, and where the energy functional $E$ is of the form

$$
E(v)=\frac{1}{2} a(v, v)+\frac{1}{2} \int_{\Omega} F\left(v^{2}(x)\right) d x,
$$

with

$$
a(u, v)=\int_{\Omega}(\mathscr{D} \nabla u) \cdot \nabla v+\int_{\Omega} V u v .
$$

In all what follows, we assume that

- $\mathscr{D} \in\left(L^{\infty}(\Omega)\right)^{d \times d} ; \mathscr{D}(x)$ is symmetric for almost all $x \in \Omega$;

$\exists \alpha>0$ such that $\xi^{T} \mathscr{D}(x) \xi \geqslant \alpha|\xi|^{2}, \forall \xi \in \mathbb{R}^{d}$ and almost all $x \in \Omega$;

- $V \in L^{p}(\Omega)$ for some $p>\max (1, d / 2)$;

- $F \in C^{1}([0,+\infty), \mathbb{R}) \cap C^{2}((0, \infty), \mathbb{R}), F^{\prime}(0)=0$ and $F^{\prime \prime}>0$ on $(0,+\infty)$;

$\exists 0 \leqslant q<2, \exists C \in \mathbb{R}_{+}$such that $\forall t \geqslant 0,\left|F^{\prime}(t)\right| \leqslant C\left(1+t^{q}\right) ;$

- $F^{\prime \prime}(t) t$ is locally bounded on $[0,+\infty)$.

To simplify the notation, we denote by $f$ the derivative of $F$. Note that there is no loss of generality in assuming in (1.4) that $f(0)=F^{\prime}(0)=0$ since the minimizers of (1.1) are not modified if $F(t)$ is replaced with $F(t)+c t$.

Problem (1.1) has exactly two minimizers $u$ and $-u$, one of them, say $u$, being positive on $\Omega$. In all what follows, $u$ will be the positive minimizer of (1.1). The function $u$ is solution to the Euler-Lagrange equation

$$
\forall v \in X, \quad\left\langle E^{\prime}(u)-\lambda u, v\right\rangle_{X^{\prime}, X}=0,
$$

for some $\lambda \in \mathbb{R}$ (the Lagrange multiplier associated with the constraint $\|u\|_{L^{2}}=1$ ) and equation (1.7), complemented with the constraint $\|u\|_{L^{2}}=1$, takes the form of the nonlinear eigenvalue problem

$$
\left\{\begin{array}{l}
A_{u} u=\lambda u \\
\|u\|_{L^{2}}=1
\end{array}\right.
$$


where for all $v \in X$,

$$
A_{v}=-\operatorname{div}(\mathscr{D} \nabla \cdot)+V+f\left(v^{2}\right)
$$

is a linear self-adjoint operator on $L^{2}(\Omega)$ with form domain $X$. Note that $E^{\prime}(u)=A_{u} u$. It can then be inferred from (1.8) that $u \in X \cap C^{0}(\bar{\Omega}), u>0$ in $\Omega$, and $\lambda$ is the ground state eigenvalue of $A_{u}$. An important point is that $\lambda$ is a simple eigenvalue of $A_{u}$. These results are classical; their proofs are recalled in Cancès et al. (2010).

We now consider a family of finite-dimensional subspaces $\left(X_{\delta}\right)_{\delta>0}$ of $X$ such that

$$
\forall v \in X, \quad \lim _{\delta \rightarrow 0} \min _{v_{\delta} \in X_{\delta}}\left\|v-v_{\delta}\right\|_{H^{1}}=0
$$

and the variational approximations of (1.1) consisting in solving

$$
I_{\delta}=\inf \left\{E\left(v_{\delta}\right), v_{\delta} \in X_{\delta}, \int_{\Omega} v_{\delta}^{2}=1\right\}
$$

Problem (1.10) has at least one minimizer $u_{\delta}$ such that $\left(u, u_{\delta}\right)_{L^{2}} \geqslant 0$, which satisfies

$$
\forall v_{\delta} \in X_{\delta}, \quad\left\langle A_{u_{\delta}} u_{\delta}, v_{\delta}\right\rangle_{X^{\prime}, X}=\lambda_{\delta}\left(u_{\delta}, v_{\delta}\right)_{L^{2}}
$$

for some $\lambda_{\delta} \in \mathbb{R}$. It is easily seen that (see, e.g., Cancès et al. (2010); Zhou (2004))

$$
\lim _{\delta \rightarrow 0}\left\|u_{\delta}-u\right\|_{H^{1}}=0
$$

or, in words, that the approximate ground state eigenfunction converges to the exact ground state eigenfunction in the $H^{1}$-norm, from which we deduce that $I_{\delta}$ and $\lambda_{\delta}$ converge to $I$ and $\lambda$, respectively, when $\delta$ goes to 0 . Optimal convergence rates have been obtained in Cancès et al. (2010) (under stronger assumptions on the nonlinearity $F$ ) for finite element and spectral Fourier discretizations.

The numerical simulation of problem (1.10) can be too costly if the approximation space $X_{\delta}$ is highdimensional. We will denote by $X_{\delta_{\mathrm{f}}}$ such a space and call it the fine discretization space. In two-grid methods, problem (1.10) is first solved in a lower-dimensional approximation space $X_{\delta_{\mathrm{c}}} \subset X_{\delta_{\mathrm{f}}}$, that we will call the coarse discretization space. Then, the so-obtained solution $u_{\delta_{\mathrm{c}}}$ is improved by solving a linearized problem in the fine discretization space $X_{\delta_{\mathrm{f}}}$. A nice feature of this approach is that, for appropriate choices of the linearized problem and of the coarse discretization space $X_{\delta_{\mathrm{c}}}$, the solution $u_{\delta_{\mathrm{f}}}^{\delta_{\mathrm{c}}}$ obtained with the two-grid method has the same accurary as the solution $u_{\delta_{\mathrm{f}}}$ obtained by solving the nonlinear problem (1.10) in the fine discretization space $X_{\delta_{\mathrm{f}}}$. Two-grid methods thus allow us to obtain the same accuracy at a much lower price. Such methods were first introduce ind Xu \& Zhou (2000) in the framework of nonlinear elliptic boundary value problems. A very nice two-grid approach is presented in Henning et al. (2014). This approach, that appears to have a better behavior as far the eigenvalues of the nonlinear system are concerned, is much more intrusive than ours and, regardless of this aspect, the evaluation of its computational cost for a given accuracy on the energy or the eigenvectors with respect to our approach needs to be further analyzed.

This article is organized as follows. In Section 2, we introduce three different two-grid algorithms to solve (1.1). In Section 3, we provide some abstract a priori error analysis for one of these algorithms. We then show how these abstract results can be applied to spectral Fourier and finite element discretizations in Sections 4 and 5 respectively. Numerical integration errors are dealt with in Section 6. Finally, 
we give several numerical examples to illustrate our theoretical results in Section 7. The class of nonlinear eigenvalue problems considered in this work is very similar to the one considered in the previous work Cancès et al. (2010) (only some assumptions on the nonlinearity $F$ will differ). For this reason, some of the proofs of the results below are simple adaptations of proofs in Cancès et al. (2010), and will therefore not be detailed for the sake of brevity. Let us mention that some of the results contained in this article have been published (in French) in the PhD thesis of the second author (Chakir (2009)).

\section{Two-grid algorithms}

Let $X_{\delta_{\mathrm{c}}}$ and $X_{\delta_{\mathrm{f}}}$ be coarse and fine discretization spaces such that $X_{\delta_{\mathrm{c}}} \subset X_{\delta_{\mathrm{f}}} \subset X$. As mentioned above, two-grid methods consist, first in computing a solution of (1.10) in a coarse discretization space $X_{\delta_{\mathrm{c}}}$ and, second in improving it by solving a linearized problem in the fine discretization space $X_{\delta_{\mathrm{f}}}$.

Several two-grid algorithms can therefore be proposed, depending on the type of linear problem we choose to solve in the fine discretization space. In the following, we introduce three of them, the first and third steps of these three schemes being the same.

1. Solve (1.10) in the coarse discretization space $X_{\delta_{\mathrm{c}}}$. Recall that the solution $u_{\delta_{\mathrm{c}}}$ of this problem is such that there exists $\lambda_{\delta_{\mathrm{c}}} \in \mathbb{R}$ such that $\left(\lambda_{\delta_{\mathrm{c}}}, u_{\delta_{\mathrm{c}}}\right)$ is also solution to the nonlinear eigenvalue problem:

$$
\left\{\begin{array}{l}
\text { find }\left(\lambda_{\delta_{\mathrm{c}}}, u_{\delta_{\mathrm{c}}}\right) \in \mathbb{R} \times X_{\delta_{\mathrm{c}}} \text { such that } \\
\forall v_{\delta_{\mathrm{c}}} \in X_{\delta_{\mathrm{c}}}, \quad\left\langle A_{u_{\delta_{\mathrm{c}}}} u_{\delta_{\mathrm{c}}}, v_{\delta_{\mathrm{c}}}\right\rangle_{X^{\prime}, X}=\lambda_{\delta_{\mathrm{c}}}\left(u_{\delta_{\mathrm{c}}}, v_{\delta_{\mathrm{c}}}\right)_{L^{2}} .
\end{array}\right.
$$

2. Two-grid scheme 1. Solve the following linear eigenvalue problem in the fine space $X_{\delta_{\mathrm{f}}}$ :

$$
\left\{\begin{array}{l}
\text { find }\left(\lambda_{\delta_{\mathrm{f}}}^{\delta_{\mathrm{c}}}, u_{\delta_{\mathrm{f}}}^{\delta_{\mathrm{c}}}\right) \in \mathbb{R} \times X_{\delta_{\mathrm{f}}} \text { such that } \\
\forall v_{\delta_{\mathrm{f}}} \in X_{\delta_{\mathrm{f}}}, \quad a\left(u_{\delta_{\mathrm{f}}}^{\delta_{\mathrm{c}}}, v_{\delta_{\mathrm{f}}}\right)+\int_{\Omega} f\left(u_{\delta_{\mathrm{c}}}^{2}\right) u_{\delta_{\mathrm{f}}}^{\delta_{\mathrm{c}}} v_{\delta_{\mathrm{f}}}=\lambda_{\delta_{\mathrm{f}}}^{\delta_{\mathrm{c}}} \int_{\Omega} u_{\delta_{\mathrm{f}}}^{\delta_{\mathrm{c}}} v_{\delta_{\mathrm{f}}}, \\
\left\|u_{\delta_{\mathrm{f}}}^{\delta_{\mathrm{f}}}\right\|_{L^{2}}=1, \quad\left(u, u_{\delta_{\mathrm{f}}}^{\delta_{\mathrm{f}}}\right)_{L^{2}} \geqslant 0 \\
\lambda_{\delta_{\mathrm{f}}}^{\delta_{\mathrm{c}}} \text { is the lowest eigenvalue of the above spectral problem. }
\end{array}\right.
$$

Two-grid scheme 2a. Solve the following linearized right-hand side problem in the fine space $X_{\delta_{\mathrm{f}}}:$

$$
\left\{\begin{array}{l}
\text { find } u_{\delta_{\mathrm{f}}}^{\delta_{\mathrm{c}}} \in X_{\delta_{\mathrm{f}}} \text { such that } \\
\forall v_{\delta_{\mathrm{f}}} \in X_{\delta_{\mathrm{f}}}, \quad a\left(u_{\delta_{\mathrm{f}}}^{\delta_{\mathrm{c}}}, v_{\delta_{\mathrm{f}}}\right)+\int_{\Omega} f\left(u_{\delta_{\mathrm{c}}}^{2}\right) u_{\delta_{\mathrm{f}}}^{\delta_{\mathrm{c}}} v_{\delta_{\mathrm{f}}}=\lambda_{\delta_{\mathrm{c}}} \int_{\Omega} u_{\delta_{\mathrm{c}}} v_{\delta_{\mathrm{f}}} .
\end{array}\right.
$$

Two-grid scheme $2 \mathbf{b}$. Solve the following linearized right-hand side problem in the fine space $\overline{X_{\delta_{\mathrm{f}}}}:$

$$
\left\{\begin{array}{l}
\text { find } u_{\delta_{\mathrm{f}}}^{\delta_{\mathrm{c}}} \in X_{\delta_{\mathrm{f}}} \text { such that } \\
\forall v_{\delta_{\mathrm{f}}} \in X_{\delta_{\mathrm{f}}}, \quad a\left(u_{\delta_{\mathrm{f}}}^{\delta_{\mathrm{c}}}, v_{\delta_{\mathrm{f}}}\right)=-\int_{\Omega} f\left(u_{\delta_{\mathrm{c}}}^{2}\right) u_{\delta_{\mathrm{c}}} v_{\delta_{\mathrm{f}}}+\lambda_{\delta_{\mathrm{c}}} \int_{\Omega} u_{\delta_{\mathrm{c}}} v_{\delta_{\mathrm{f}}} .
\end{array}\right.
$$

3. Compute the Rayleigh quotient $\tilde{\lambda}_{\delta_{\mathrm{f}}}^{\delta_{\mathrm{c}}}$ for $u_{\delta_{\mathrm{f}}}^{\delta_{\mathrm{c}}}$ :

$$
\tilde{\lambda} \delta_{\delta_{\mathrm{f}}}^{\delta_{\mathrm{c}}}=\frac{\left\langle A_{u_{\delta_{\mathrm{f}}}} u_{\delta_{\mathrm{f}}}^{\delta_{\mathrm{c}}}, u_{\delta_{\mathrm{f}}}^{\delta_{\mathrm{c}_{\mathrm{c}}}}\right\rangle_{X^{\prime}, X}}{\left\|u_{\delta_{\mathrm{f}}}^{\delta_{\mathrm{c}}}\right\|_{L^{2}}^{2}}
$$


In the limit $\delta_{\mathrm{f}}=0$ (that corresponds to $X_{\delta_{\mathrm{f}}}=X$ ), the second step of scheme 1 amounts to computing the ground state $\left(\lambda_{0}^{\delta_{\mathrm{c}}}, u_{0}^{\delta_{\mathrm{c}}}\right)$ of the self-adjoint operator $A_{u_{\delta_{\mathrm{c}}}}$, while schemes $2 \mathrm{a}$ and $2 \mathrm{~b}$ amount to solving the boundary value problems $A_{u_{\delta_{\mathrm{c}}}} u_{0}^{\delta_{\mathrm{c}}}=\lambda_{\delta_{\mathrm{c}}} u_{\delta_{\mathrm{c}}}$ and $A_{0} u_{0}^{\delta_{\mathrm{c}}}=\left(\lambda_{\delta_{\mathrm{c}}}-f\left(u_{\delta_{\mathrm{c}}}^{2}\right)\right) u_{\delta_{\mathrm{c}}}$, respectively.

In this paper, we shall focus on the analysis of the first scheme, both from the theoretical and simulation points of view. The analysis of the other two schemes will be the matter of a forthcoming work. Let us just mention here that on preliminary simulations, schemes $2 \mathrm{a}$ and $2 \mathrm{~b}$ provide similar results as scheme 1 (see Chakir (2009)).

\section{Abstract error analysis of scheme 1}

We denote by $u$ the unique positive solution of (1.1), by $u_{\delta_{\mathrm{c}}}$ a minimizer of the discretized nonlinear problem (1.10) such that $\left(u, u_{\delta_{\mathrm{c}}}\right)_{L^{2}} \geqslant 0$, and by $u_{\delta_{\mathrm{f}}}^{\delta_{\mathrm{c}}}$ the approximation of $u$ computed with scheme 1 . The aim of this section is to establish error bounds on $\left\|u-u_{\delta_{\mathrm{f}}}^{\delta_{\mathrm{c}}}\right\|_{H^{1}},\left\|u-u_{\delta_{\mathrm{f}}}^{\delta_{\mathrm{f}}}\right\|_{L^{2}}$ and $\left|\lambda-\lambda_{\delta_{\mathrm{f}}}^{\delta_{\mathrm{c}}}\right|$, in the general framework of assumptions (1.2)-(1.6) and (1.9).

\subsection{Preliminaries}

Our analysis relies on the introduction of the solution $u_{0}^{\delta_{\mathrm{c}}}$ of the two-grid scheme in the limiting case when $\delta_{\mathrm{f}}=0$ (that is for $X_{\delta_{\mathrm{f}}}=X$ ). Recall that $u_{0}^{\delta_{\mathrm{c}}}$ is the positive ground state eigenfunction of $A_{u_{\delta_{\mathrm{c}}}}$. We denote by $\lambda_{0}^{\delta_{\mathrm{c}}}$ the associated eigenvalue. The minmax principle gives

$$
\lambda_{0}^{\delta_{\mathrm{c}}}=\inf \left\{\left\langle A_{u_{\delta_{\mathrm{c}}}} v, v\right\rangle_{X^{\prime}, X}, v \in X, \int_{\Omega} v^{2}=1\right\}
$$

and the solution $\left(\lambda_{\delta_{\mathrm{f}}}^{\delta_{\mathrm{c}}}, u_{\delta_{\mathrm{f}}}^{\delta_{\mathrm{c}}}\right)$ provided by the two-grid scheme 1 can then be interpreted as the solution of the variational approximation

$$
\lambda_{\delta_{\mathrm{f}}}^{\delta_{\mathrm{c}}}=\inf \left\{\left\langle A_{u_{\delta_{\mathrm{c}}}} v_{\delta_{\mathrm{f}}}, v_{\delta_{\mathrm{f}}}\right\rangle_{X^{\prime}, X}, v_{\delta_{\mathrm{f}}} \in X_{\delta_{\mathrm{f}}}, \int_{\Omega} v_{\delta_{\mathrm{f}}}^{2}=1\right\}
$$

of problem (3.1) in the discretization space $X_{\delta_{\mathrm{f}}}$.

Problem (3.2) has at least one minimizer $u_{\delta_{\mathrm{f}}}^{\delta_{\mathrm{c}}}$, which satisfies (2.1), for some $\lambda_{\delta_{\mathrm{f}}}^{\delta_{\mathrm{c}}} \in \mathbb{R}$. Note that, when $\delta_{\mathrm{f}}=\delta_{\mathrm{c}}, u_{\delta_{\mathrm{c}}}^{\delta_{\mathrm{c}}}=u_{\delta_{\mathrm{c}}}$ is solution to (3.2).

The following numerical analysis relies on the properties of the mapping $v \mapsto\left(\lambda_{v}, z_{v}\right)$, where $\lambda_{v}$ denotes the lowest eigenvalue of the self-adjoint operator $A_{v}$ and $z_{v}>0$ the associated positive normalized eigenfunction:

$$
\left\{\begin{array}{l}
A_{v} z_{v}=\lambda_{v} z_{v}, \\
z_{v}>0, \\
\left\|z_{v}\right\|_{L^{2}}=1 .
\end{array}\right.
$$

The function $z_{v}$ is also the minimizer of the problem

$$
\inf \left\{\left\langle A_{v} w, w\right\rangle_{X^{\prime}, X}, w \in X, \int_{\Omega} w^{2}=1\right\},
$$

(which amounts to minimizing the Rayleigh quotient associated with the self-adjoint operator $A_{v}$ ). In the special case when $v=u$, we have $\left(\lambda_{u}, z_{u}\right)=(\lambda, u)$. 
The following technical lemmas will be used throughout the article. For the reader's convenience, we first state all the lemmas, and postpone their proofs until the end of the section.

Lemma 3.1 Under assumptions (1.2), there exist $\beta_{0} \in \mathbb{R}_{+}$and $M \in \mathbb{R}_{+}$such that

$$
\forall w \in X, \quad \frac{\alpha}{2}\|\nabla w\|_{L^{2}}^{2}-\beta_{0}\|w\|_{L^{2}}^{2} \leqslant a(w, w) \leqslant M\|w\|_{H^{1}}^{2} .
$$

We recall that $f$ denotes the derivative of $F$ and $u$ the unique positive minimizer of (1.1) that satisfies $A_{u} u=\lambda u$ with $\lambda \in \mathbb{R}$.

Lemma 3.2 Assume that $F$ satisfies assumptions (1.4)-(1.6). Denoting by $r=\frac{6}{5-2 q}\left(\frac{6}{5} \leqslant r<6\right)$, there exists a constant $C \in \mathbb{R}_{+}$such that for all $(v, w, z) \in X^{3}$,

$$
\begin{aligned}
\left|\int_{\Omega} f\left(v^{2}\right) w z\right| & \leqslant C\left(1+\|v\|_{L^{6}}^{2 q}\right)\|w\|_{L^{6}}\|z\|_{L^{r}}, \\
\left|\int_{\Omega}\left(f\left(v^{2}\right)-f\left(u^{2}\right)\right) u w\right| & \leqslant C\left(1+\|v\|_{L^{6}}^{2 q}\right)\|w\|_{L^{6}}\|u-v\|_{L^{r}}, \\
\left|\int_{\Omega}\left(f\left(v^{2}\right)-f\left(u^{2}\right)\right) v^{2}\right| & \leqslant C\left(1+\|v\|_{L^{6}}^{2 q+1}\right)\|u-v\|_{L^{r}}, \\
0 \leqslant \int_{\Omega} F\left(v^{2}\right)-F\left(u^{2}\right)-f\left(u^{2}\right)\left(v^{2}-u^{2}\right) & \leqslant C\left(1+\|v\|_{L^{6}}^{2 q+1}\right)\|u-v\|_{L^{r}} .
\end{aligned}
$$

Besides, in the case where $X=H_{\#}^{1}(\Omega)$, there exists $C \in \mathbb{R}_{+}$such that for all $(v, w) \in X^{2}$,

$$
\int_{\Omega}\left(f\left(u^{2}\right)-f\left(v^{2}\right)\right) w^{2} \leqslant C \int_{\Omega} \mathbb{1}_{u \geqslant|v|}(u-v) w^{2} \quad\left(X=H_{\#}^{1}(\Omega) \text { only }\right){ }^{1}
$$

while, in the case where $X=H_{0}^{1}(\Omega)$, for all $\varepsilon>0$, there exists $C_{\varepsilon} \in \mathbb{R}_{+}$such that for all $(v, w) \in X^{2}$,

$$
\int_{\Omega}\left(f\left(u^{2}\right)-f\left(v^{2}\right)\right) w^{2} \leqslant \varepsilon\|w\|_{L^{2}}^{2}+C_{\varepsilon} \int_{\Omega} \mathbb{1}_{u \geqslant|v|}(u-v) w^{2} \quad\left(X=H_{0}^{1}(\Omega) \text { only }\right)
$$

LEMma 3.3 There exist $0<M_{2} \leqslant M_{1}<\infty$ such that

$$
\forall v \in X, \quad 0 \leqslant\left\langle\left(A_{u}-\lambda\right) v, v\right\rangle_{X^{\prime}, X} \leqslant M_{1}\|v\|_{H^{1}}^{2}
$$

and

$$
\forall v \in u^{\perp}:=\left\{v \in X,(u, v)_{L^{2}}=0\right\}, \quad M_{2}\|v\|_{H^{1}}^{2} \leqslant\left\langle\left(A_{u}-\lambda\right) v, v\right\rangle_{X^{\prime}, X} .
$$

Moreover, there exists $\gamma>0$ such that, for all $w \in X$ such that $\|w\|_{L^{2}}=1$ and $(u, w)_{L^{2}} \geqslant 0$,

$$
\gamma\|w-u\|_{H^{1}}^{2} \leqslant\left\langle\left(A_{u}-\lambda\right)(w-u),(w-u)\right\rangle_{X^{\prime}, X} .
$$

The properties of the ground state eigenpair $\left(\lambda_{v}, z_{v}\right)$ of $A_{v}$ are collected in the following lemma.

Lemma 3.4 There exists a constant $C \in \mathbb{R}_{+}$such that

$$
\begin{gathered}
\forall v \in X, \quad\left|\lambda_{v}\right|+\left\|z_{v}\right\|_{H^{1}}^{2} \leqslant C\left(1+\|v\|_{L^{6}}^{2 q}\right), \\
\forall v \in X, \quad\left\|z_{v}-u\right\|_{H^{1}} \leqslant C\left(1+\|v\|_{L^{6}}^{2 q}\right)\|u-v\|_{L^{\max (r, 2)}} .
\end{gathered}
$$

In addition,

\footnotetext{
${ }^{1}$ The notation $\mathbb{1}_{\mathscr{P}}$ stands for the characteristic function of the set where the property $\mathscr{P}$ holds.
} 
- in the case when $X=H_{\#}^{1}(\Omega)$, there exists $C \in \mathbb{R}_{+}$such that

$$
\forall v \in X, \quad\left|\lambda_{v}-\lambda\right| \leqslant C\left(1+\|v\|_{L^{6}}^{2 q}\right)\|v-u\|_{L^{\max (r, 2)}} \quad\left(X=H_{\#}^{1}(\Omega) \text { only }\right)
$$

- in the case when $X=H_{0}^{1}(\Omega)$, there exists, for any $\varepsilon>0$, a constant $C_{\varepsilon} \in \mathbb{R}_{+}$such that

$$
\forall v \in X, \quad\left|\lambda_{v}-\lambda_{u}\right| \leqslant 2 \varepsilon+C_{\varepsilon}\left(1+\|v\|_{L^{6}}^{2 q}\right)\|u-v\|_{L^{\max (r, 2)}} \quad\left(X=H_{0}^{1}(\Omega) \text { only }\right)
$$

Estimates (3.15)-(3.16) are sufficient for our purpose, but are not optimal; refined estimates are actually given in the proof of Lemma 3.4 .

For all $v \in X$, we denote by $\lambda_{2, v}$ the second eigenvalue of $A_{v}$. Since $\lambda=\lambda_{u}$ is a simple eigenvalue of $A_{u}$, there is a gap, denoted by $g=\lambda_{2, u}-\lambda_{u}>0$, between the first and second eigenvalues of $A_{u}$.

LEMMA 3.5 There exists $0<\eta \leqslant 1$ such that for all $v \in X$ such that $\|v-u\|_{H^{1}} \leqslant \eta$, we have $\lambda_{2, v}-\lambda_{v} \geqslant$ $g / 2$.

Proposition 3.1 There exist $\eta>0$ and $0<c_{0} \leqslant C_{0}<\infty$ such that for all $v \in X$ such that $\|v-u\|_{H^{1}} \leqslant \eta$ and all $w \in X$ such that $\|w\|_{L^{2}}=1$ and $\left(z_{v}, w\right)_{L^{2}} \geqslant 0$, we have

$$
c_{0}\left\|w-z_{v}\right\|_{H^{1}}^{2} \leqslant\left\langle\left(A_{v}-\lambda_{v}\right)\left(w-z_{v}\right),\left(w-z_{v}\right)\right\rangle_{X^{\prime}, X} \leqslant C_{0}\left\|w-z_{v}\right\|_{H^{1}}^{2} .
$$

Proof of Lemma 3.1. For brevity, we only explain in detail the arguments for $d=3$, in which case $p>3 / 2$. Under assumptions (1.2), there exists a positive constant $M$ such that

$$
\forall w \in X, \quad a(w, w) \leqslant\|\mathscr{D}\|_{L^{\infty}}\|\nabla w\|_{L^{2}}^{2}+\|V\|_{L^{p}}\|w\|_{L^{2 p^{\prime}}}^{2} \leqslant M\|w\|_{H^{1}}^{2}
$$

where $1 \leqslant p^{\prime}=\left(1-p^{-1}\right)^{-1}<3$. Using Hölder's inequality, we have that for any $w \in X$,

$$
\begin{aligned}
a(w, w) & =\int_{\Omega} \mathscr{D} \nabla w \cdot \nabla w+\int_{\Omega} V w^{2} \\
& \geqslant \alpha\|\nabla w\|_{L^{2}}^{2}-\|V\|_{L^{p}}\|w\|_{L^{2}}^{2-3 / p}\|w\|_{L^{6}}^{3 / p} \\
& \geqslant \alpha\|\nabla w\|_{L^{2}}^{2}-C_{6}^{3 / p}\|V\|_{L^{p}}\|w\|_{L^{2}}^{2-3 / p}\|w\|_{H^{1}}^{3 / p},
\end{aligned}
$$

where $C_{6}$ is the Sobolev constant such that for all $v \in X,\|v\|_{L^{6}} \leqslant C_{6}\|v\|_{H^{1}}$.

Using Young's inequality, we have for all $\varepsilon>0$ and $w \in X$,

$$
\begin{aligned}
C_{6}^{3 / p}\|V\|_{L^{p}}\|w\|_{L^{2}}^{2-3 / p}\|w\|_{H^{1}}^{3 / p} & =\left(\frac{1}{\varepsilon} C_{6}^{3 / p}\|V\|_{L^{p}}\|w\|_{L^{2}}^{2-3 / p}\right)\left(\varepsilon\|w\|_{H^{1}}^{3 / p}\right) \\
& \leqslant \frac{2 p-3}{2 p}\left(\frac{1}{\varepsilon} C_{6}^{3 / p}\|V\|_{L^{p}}\|w\|_{L^{2}}^{2-3 / p}\right)^{2 p /(2 p-3)}+\frac{3}{2 p}\left(\varepsilon\|w\|_{H^{1}}^{3 / p}\right)^{2 p / 3} \\
& =\frac{2 p-3}{2 p}\left(\left(\frac{1}{\varepsilon} C_{6}^{3 / p}\|V\|_{L^{p}}\right)^{2 p / 3}\right)^{3 /(2 p-3)}\|w\|_{L^{2}}^{2}+\frac{3}{2 p} \varepsilon^{2 p / 3}\|w\|_{H^{1}}^{2} .
\end{aligned}
$$

Choosing $\frac{3}{2 p} \varepsilon^{2 p / 3}=\frac{\alpha}{2}$, we get

$$
C_{6}^{3 / p}\|V\|_{L^{p}}\|w\|_{L^{2}}^{2-3 / p}\|w\|_{H^{1}}^{3 / p} \leqslant \frac{2 p-3}{2 p}\left(\frac{3}{p \alpha} C_{6}^{2}\|V\|_{L^{p}}^{2 p / 3}\right)^{3 /(2 p-3)}\|w\|_{L^{2}}^{2}+\frac{\alpha}{2}\|w\|_{L^{2}}^{2}+\frac{\alpha}{2}\|\nabla w\|_{L^{2}}^{2},
$$


which implies

$$
a(w, w) \geqslant \frac{\alpha}{2}\|\nabla w\|_{L^{2}}^{2}-\left(\frac{2 p-3}{2 p}\left(\frac{3}{p \alpha} C_{6}^{2}\|V\|_{L^{p}}^{2 p / 3}\right)^{3 /(2 p-3)}+\frac{\alpha}{2}\right)\|w\|_{L^{2}}^{2} .
$$

Hence, there exists a positive constant $\beta_{0}$ such that

$$
\forall w \in X, \quad a(w, w) \geqslant \frac{\alpha}{2}\|\nabla w\|_{L^{2}}^{2}-\beta_{0}\|w\|_{L^{2}}^{2} .
$$

This completes the proof.

Proof of Lemma 3.2. In this proof, $C$ denotes a non-negative constant independent on $v, w$ and $z$, but whose value is allowed to change from one line to another. We recall that $r=\frac{6}{5-2 q}$.

Proof of (3.4). It follows from assumption (1.5) that for all $(v, w, z) \in X^{3}$,

$$
\left|\int_{\Omega} f\left(v^{2}\right) w z\right| \leqslant C \int_{\Omega}\left(1+|v|^{2 q}\right)|w||z| \leqslant C\left(1+\|v\|_{L^{6}}^{2 q}\right)\|w\|_{L^{6}}\|z\|_{L^{r}}
$$

Proof of (3.5). We first write

$$
\int_{\Omega}\left(f\left(v^{2}\right)-f\left(u^{2}\right)\right) u w=\int_{\Omega} \widetilde{w}_{v, u}(u-v) w, \quad \text { with } \quad \widetilde{w}_{v, u}=-\frac{f\left(u^{2}\right)-f\left(v^{2}\right)}{u-v} u .
$$

Since $u \in L^{\infty}(\Omega)$ and $u \geqslant 0$, it holds

$$
\left|\widetilde{w}_{v, u}\right| \leqslant \mid \begin{array}{ll}
2\left(f\left(v^{2}\right)+f\left(\|u\|_{L^{\infty}}^{2}\right)\right), & \text { when }|v|<u / 2 \\
4 \sup _{t \in\left(0,4\|u\|_{L^{\infty}}^{2} F^{\prime \prime}(t) t,\right.} & \text { when } u / 2 \leqslant|v|<2 u \\
\left|f\left(v^{2}\right)\right|+f\left(\|u\|_{L^{\infty}}^{2}\right), & \text { when }|v| \geqslant 2 u
\end{array}
$$

The above estimate is easily obtained in the case when $|v|<u / 2$ or $|v| \geqslant 2 u$. When $u / 2 \leqslant|v|<2 u$, we observe that

$$
\left|f\left(u^{2}\right)-f\left(v^{2}\right)\right|=\left|\int_{u^{2}}^{v^{2}} F^{\prime \prime}(t) d t\right|=\left|\int_{u^{2}}^{v^{2}} \frac{t F^{\prime \prime}(t)}{t} d t\right| \leqslant 2\left(\sup _{t \in\left(0,4\|u\|_{L^{\infty}}\right.} F^{\prime \prime}(t) t\right)|\ln u-\ln | v|| .
$$

It follows that when $u / 2 \leqslant|v|<2 u$, there exists $u / 2 \leqslant \xi<2 u$ such that

$$
\left|\widetilde{w}_{v, u}\right| \leqslant 2\left(\sup _{t \in\left(0,4\|u\|_{L^{\infty}}^{2}\right]} F^{\prime \prime}(t) t\right)\left|\frac{\ln u-\ln v}{u-v}\right| u=2\left(\sup _{t \in\left(0,4\|u\|_{L^{\infty}}^{2}\right]} F^{\prime \prime}(t) t\right) \frac{u}{\xi} \leqslant 4 \sup _{t \in\left(0,4\|u\|_{L^{\infty}}^{2}\right]} F^{\prime \prime}(t) t .
$$

Thus, (3.19) is proved. This estimate, together with assumptions (1.5) and (1.6), yields

$$
\left|\widetilde{w}_{v, u}\right| \leqslant C\left(1+|v|^{2 q}\right),
$$

which, combined with (3.18), straightforwardly leads to (3.5).

Proof of (3.6). For all $v \in X$, we can write

$$
\int_{\Omega}\left(f\left(v^{2}\right)-f\left(u^{2}\right)\right) v^{2}=\int_{\Omega} w_{v, u}(v-u), \quad \text { with } \quad w_{v, u}=v^{2} \frac{f\left(v^{2}\right)-f\left(u^{2}\right)}{v-u} .
$$


As $u \in L^{\infty}(\Omega)$, we have (see the proof of (Cancès et al., 2010, Theorem 1))

$$
\left|w_{v, u}\right| \leqslant \mid \begin{array}{ll}
12 u \sup _{t \in\left(0,4\|u\|_{L^{\infty}}^{2} F^{\prime \prime}(t) t,\right.} & \text { when }|v|<2 u, \\
2\left(\left|f\left(v^{2}\right)\right|+f\left(\|u\|_{L^{\infty}}^{2}\right)\right)|v|, & \text { when }|v| \geqslant 2 u .
\end{array}
$$

We infer from assumptions (1.5) and (1.6) that

$$
\left|w_{v, u}\right| \leqslant C\left(1+|v|^{2 q+1}\right) .
$$

Putting together (3.20) and (3.21), we obtain

$$
\left|\int_{\Omega}\left(f\left(v^{2}\right)-f\left(u^{2}\right)\right) v^{2}\right| \leqslant\left\|w_{v, u}\right\|_{L^{6 /(1+2 q)}}\|u-v\|_{L^{r}} \leqslant C\left(1+\|v\|_{L^{6}}^{2 q+1}\right)\|u-v\|_{L^{r}} .
$$

Proof of (3.7). The left-hand side inequality in (3.7) follows from the convexity of $F$ (assumption (1.4)). On the other hand,

$$
\forall v \in X, \quad \int_{\Omega} F\left(v^{2}\right)-F\left(u^{2}\right)-f\left(u^{2}\right)\left(v^{2}-u^{2}\right)=\int_{\Omega}\left(f(\xi)-f\left(u^{2}\right)\right)\left(v^{2}-u^{2}\right)
$$

with $\xi \in\left[\min \left(u^{2}, v^{2}\right), \max \left(u^{2}, v^{2}\right)\right]$. Using assumption (1.5) and the boundedness of $u$, we get

$$
\int_{\Omega} F\left(v^{2}\right)-F\left(u^{2}\right)-f\left(u^{2}\right)\left(v^{2}-u^{2}\right) \leqslant C \int_{\Omega}\left(1+|v|^{2 q+1}\right)|u-v| \leqslant C\left(1+\|v\|_{L^{6}}^{2 q+1}\right)\|u-v\|_{L^{r}} .
$$

Proof of (3.8). We assume here that $X=H_{\#}^{1}(\Omega)$. Since $u$ is continuous, everywhere positive, and periodic, there exists a positive constant $\alpha_{0}$ such that $u \geqslant \alpha_{0}>0$.

Denoting by $\Omega_{-}=\left\{x \in \Omega|| v(x) \mid<\frac{\alpha_{0}}{2}\right\}$ and $\Omega_{+}=\left\{x \in \Omega|| v(x) \mid \geqslant \frac{\alpha_{0}}{2}\right\}$, we have

$$
\forall(v, w) \in X^{2}, \quad \int_{\Omega}\left(f\left(u^{2}\right)-f\left(v^{2}\right)\right) w^{2}=\int_{\Omega_{-}}\left(f\left(u^{2}\right)-f\left(v^{2}\right)\right) w^{2}+\int_{\Omega_{+}}\left(f\left(u^{2}\right)-f\left(v^{2}\right)\right) w^{2} .
$$

Since, over $\Omega_{-}, v$ is such that $|v|<u \leqslant\|u\|_{L^{\infty}}$ and $f$ is monotonically increasing, we have $\left|f\left(v^{2}\right)\right| \leqslant$ $\left|f\left(\|u\|_{L^{\infty}}^{2}\right)\right|$ over $\Omega_{-}$, so that

$$
\begin{aligned}
\int_{\Omega_{-}}\left(f\left(u^{2}\right)-f\left(v^{2}\right)\right) w^{2} & \leqslant 2 f\left(\|u\|_{L^{\infty}}^{2}\right) \int_{\Omega_{-}} \frac{u-v}{u-v} w^{2} \\
& \leqslant \frac{4 f\left(\|u\|_{L^{\infty}}^{2}\right)}{\alpha_{0}} \int_{\Omega_{-}}(u-v) w^{2} \\
& \leqslant C \int_{\Omega_{-}}(u-v) w^{2}
\end{aligned}
$$

Denoting by $\Omega_{+}^{1}=\left\{x \in \Omega|u(x)>| v(x) \mid \geqslant \frac{\alpha_{0}}{2}\right\}$ and $\Omega_{+}^{2}=\left\{x \in \Omega|| v(x) \mid \geqslant u(x) \geqslant \alpha_{0}\right\}$, and using the fact that $f$ is monotonically increasing, we obtain

$$
\begin{aligned}
\int_{\Omega_{+}}\left(f\left(u^{2}\right)-f\left(v^{2}\right)\right) w^{2} & =\int_{\Omega_{+}^{1}}\left(f\left(u^{2}\right)-f\left(v^{2}\right)\right) w^{2}+\int_{\Omega_{+}^{2}}\left(f\left(u^{2}\right)-f\left(v^{2}\right)\right) w^{2} \\
& \leqslant \int_{\Omega_{+}^{1}}\left(f\left(u^{2}\right)-f\left(v^{2}\right)\right) w^{2} \\
& =\int_{\Omega_{+}^{1}} f^{\prime}(\xi)\left(u^{2}-v^{2}\right) w^{2}
\end{aligned}
$$


where $\xi \in\left[v^{2}, u^{2}\right] \subset\left[\alpha_{0}^{2} / 4,\|u\|_{L^{\infty}}^{2}\right]$. Since $F \in C^{2}((0, \infty), \mathbb{R})$ (assumption (1.4)), we get

$$
\int_{\Omega_{+}}\left(f\left(u^{2}\right)-f\left(v^{2}\right)\right) w^{2} \leqslant C \int_{\Omega_{+}^{1}}\left(u^{2}-v^{2}\right) w^{2} \leqslant C \int_{\Omega_{+}^{1}} 2 u(u-v) w^{2} \leqslant C \int_{\Omega_{+}^{1}}(u-v) w^{2} .
$$

Combining (3.22), (3.23), and (3.24), we obtain (3.8).

Proof of (3.9). We now consider the case when $X=H_{0}^{1}(\Omega)$. Since $F \in C^{1}([0,+\infty), \mathbb{R})$ and $f(0)=0$, there exists, for any $\varepsilon>0$, a constant $\beta_{\varepsilon}>0$ such that for all $0 \leqslant t \leqslant \beta_{\varepsilon}^{2}$,

$$
|f(t)| \leqslant \varepsilon .
$$

Since $f$ is monotonically increasing, we have for all $(v, w) \in X^{2}$,

$$
\begin{aligned}
\int_{\Omega}\left(f\left(u^{2}\right)-f\left(v^{2}\right)\right) w^{2} & =\int_{\Omega} \mathbb{1}_{u \leqslant|v|}\left(f\left(u^{2}\right)-f\left(v^{2}\right)\right) w^{2}+\int_{\Omega} \mathbb{1}_{u>|v|}\left(f\left(u^{2}\right)-f\left(v^{2}\right)\right) w^{2} \\
& \leqslant \int_{\Omega} \mathbb{1}_{u>|v|}\left(f\left(u^{2}\right)-f\left(v^{2}\right)\right) w^{2} .
\end{aligned}
$$

Denoting by $\Omega_{1, \varepsilon}=\left\{x \in \Omega|0 \leqslant| v(x) \mid<u(x)<\beta_{\varepsilon}\right\}, \Omega_{2, \varepsilon}=\left\{x \in \Omega|0 \leqslant| v(x) \mid<\beta_{\varepsilon} / 2, u(x) \geqslant \beta_{\varepsilon}\right\}$, and $\Omega_{3, \varepsilon}=\left\{x \in \Omega\left|\beta_{\varepsilon} / 2<\right| v(x) \mid<u(x)\right\}$, we split the right-hand side of (3.26) into three parts. Using (3.25) and the boundedness of $u$, we get

$$
\int_{\Omega_{1, \varepsilon}}\left(f\left(u^{2}\right)-f\left(v^{2}\right)\right) w^{2} \leqslant 2 \varepsilon\|w\|_{L^{2}}^{2},
$$

and

$$
\int_{\Omega_{2, \varepsilon}}\left(f\left(u^{2}\right)-f\left(v^{2}\right)\right) w^{2} \leqslant \frac{4}{\beta_{\varepsilon}} f\left(\|u\|_{L^{\infty}}^{2}\right) \int_{\Omega_{2, \varepsilon}}(u-v) w^{2} .
$$

We then note that there exists $\xi$ with $v^{2} \leqslant \xi \leqslant u^{2}$ such that

$$
\int_{\Omega_{3, \varepsilon}}\left(f\left(u^{2}\right)-f\left(v^{2}\right)\right) w^{2}=\int_{\Omega_{3, \varepsilon}} f^{\prime}(\xi)\left(u^{2}-v^{2}\right) w^{2} \leqslant 2\left(\max _{t \in\left[\beta_{\varepsilon}^{2} / 4,\|u\|_{L^{\infty}}^{2}\right]} F^{\prime \prime}(t)\right)\|u\|_{L^{\infty}} \int_{\Omega_{3, \varepsilon}}(u-v) w^{2} .
$$

Thus, (3.9) is proved with $C_{\varepsilon}=\frac{4}{\beta_{\varepsilon}} f\left(\|u\|_{L^{\infty}}^{2}\right)+2\left(\max _{t \in\left[\beta_{\varepsilon}^{2} / 4,\|u\|_{L^{\infty}}^{2}\right]} F^{\prime \prime}(t)\right)\|u\|_{L^{\infty}}$.

Proof of Lemma 3.3. The detailed proof of (3.10) and (3.11) can be found in Cancès et al. (2010). Let us prove (3.12). We know from inequality (20) in Cancès et al. (2010) that there exists $\eta>0$ such that

$$
\forall v \in X, \quad\left\langle\left(A_{u}-\lambda\right) v, v\right\rangle_{X^{\prime}, X} \geqslant \eta\left(\|v\|_{L^{2}}^{2}-\left|(u, v)_{L^{2}}\right|^{2}\right) \geqslant 0 .
$$

Since $\|w\|_{L^{2}}=1$ and $\|u\|_{L^{2}}=1$, we have

$$
\|w-u\|_{L^{2}}^{2}-\left|(w-u, u)_{L^{2}}\right|^{2} \geqslant\|w-u\|_{L^{2}}^{2}-\left(1-(w, u)_{L^{2}}\right)=\frac{1}{2}\|w-u\|_{L^{2}}^{2},
$$

which together with (3.27) implies

$$
\left\langle\left(A_{u}-\lambda\right)(w-u),(w-u)\right\rangle_{X^{\prime}, X} \geqslant \frac{\eta}{2}\|w-u\|_{L^{2}}^{2} .
$$


In view of inequality (22) in Cancès et al. (2010), there exists a constant $C \in \mathbb{R}_{+}$such that

$$
\left\langle\left(A_{u}-\lambda\right)(w-u),(w-u)\right\rangle_{X^{\prime}, X} \geqslant \frac{\alpha}{2}\|\nabla(w-u)\|_{L^{2}}^{2}-C\|w-u\|_{L^{2}}^{2} .
$$

We obtain (3.12) with $\gamma=\frac{\alpha \eta}{2(\eta+2 C)}$ by combining (3.28) and (3.29).

Proof of Lemma 3.4. In this proof, $C, C_{1}, C_{2}$ denote non-negative constants independent of $v$, but whose values are allowed to change from one line to another. As $\lambda_{v}$ is the lowest eigenvalue of $A_{v}$, we infer from (1.4), (3.3) and the boundedness of $u$ that

$$
\begin{aligned}
\lambda_{v}=\left\langle A_{v} z_{v}, z_{v}\right\rangle_{X^{\prime}, X} & \leqslant\left\langle A_{v} u, u\right\rangle_{X^{\prime}, X}=a(u, u)+\int_{\Omega} f\left(v^{2}\right) u^{2} \\
& \leqslant M\|u\|_{H^{1}}^{2}+C\left(1+\|v\|_{L^{2 q}}^{2 q}\right) \leqslant C\left(1+\|v\|_{L^{2 q}}^{2 q}\right) .
\end{aligned}
$$

Using (3.3), the fact that $\left\|z_{v}\right\|_{L^{2}}=1$, and the positivity of $F^{\prime \prime}(t)$ (which implies that $f\left(t^{2}\right) \geqslant 0$ for all $t \in \mathbb{R})$, we obtain

$$
\lambda_{v}=a\left(z_{v}, z_{v}\right)+\int_{\Omega} f\left(v^{2}\right) z_{v}^{2} \geqslant \frac{\alpha}{2}\left\|z_{v}\right\|_{H^{1}}^{2}-\frac{\alpha}{2}-\beta_{0}
$$

which, together with (3.30), readily leads to (3.13).

We now turn to the proof of (3.15) and (3.16). Let $v \in X$. We shall analyze each case of the alternative $\lambda_{v}>\lambda=\lambda_{u}$ or $\lambda_{v} \leqslant \lambda=\lambda_{u}$. In the former case, since $\lambda_{v}$ is the lowest eigenvalue of $A_{v}$, we have

$$
\lambda<\lambda_{v}=\left\langle A_{v} z_{v}, z_{v}\right\rangle_{X^{\prime}, X} \leqslant\left\langle A_{v} u, u\right\rangle_{X^{\prime}, X}=\lambda+\int_{\Omega}\left(f\left(v^{2}\right)-f\left(u^{2}\right)\right) u^{2} .
$$

In the latter case, we use this time the fact that $\lambda_{u}$ is the lowest eigenvalue of $A_{u}$ to get

$$
\lambda_{v} \leqslant \lambda=\left\langle A_{u} u, u\right\rangle_{X^{\prime}, X} \leqslant\left\langle A_{u} z_{v}, z_{v}\right\rangle_{X^{\prime}, X}=\lambda_{v}+\int_{\Omega}\left(f\left(u^{2}\right)-f\left(v^{2}\right)\right) z_{v}^{2} .
$$

Therefore, using either (3.5) with $w=u$ (former case), or (3.8)-(3.9) with $w=z_{v}$ and (3.13) (latter case), we obtain that, for all $v \in X$,

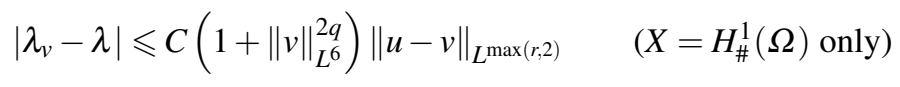

and

$$
\left|\lambda_{v}-\lambda\right| \leqslant 2 \varepsilon+C_{\varepsilon}\left(1+\|v\|_{L^{6}}^{2 q}\right)\|u-v\|_{L^{\max (r, 2)}} \quad\left(X=H_{0}^{1}(\Omega) \text { only }\right) .
$$

Since $\lambda_{v} z_{v}-\lambda_{u} u=A_{v} z_{v}-A_{u} u$, we have

$$
\begin{aligned}
\left(\lambda_{v} z_{v}-\lambda_{u} u, z_{v}-u\right) & =\left\langle A_{v} z_{v}-A_{u} u, z_{v}-u\right\rangle_{X^{\prime}, X} \\
& =a\left(z_{v}-u, z_{v}-u\right)+\int_{\Omega} f\left(v^{2}\right) z_{v}\left(z_{v}-u\right)-\int_{\Omega} f\left(u^{2}\right) u\left(z_{v}-u\right) \\
& =a\left(z_{v}-u, z_{v}-u\right)+\int_{\Omega} f\left(u^{2}\right)\left(z_{v}-u\right)^{2}+\int_{\Omega}\left(f\left(v^{2}\right)-f\left(u^{2}\right)\right) z_{v}\left(z_{v}-u\right) \\
& =\left\langle A_{u}\left(z_{v}-u\right),\left(z_{v}-u\right)\right\rangle_{X^{\prime}, X}+\int_{\Omega}\left(f\left(v^{2}\right)-f\left(u^{2}\right)\right) z_{v}\left(z_{v}-u\right) .
\end{aligned}
$$


Hence, we have

$$
\left\langle\left(A_{u}-\lambda_{u}\right)\left(z_{v}-u\right),\left(z_{v}-u\right)\right\rangle_{X^{\prime}, X}=\left(\lambda_{v}-\lambda_{u}\right) \int_{\Omega} z_{v}\left(z_{v}-u\right)+\int_{\Omega}\left(f\left(u^{2}\right)-f\left(v^{2}\right)\right) z_{v}\left(z_{v}-u\right),
$$

which together with (3.12) implies that

$$
\begin{aligned}
\gamma\left\|z_{v}-u\right\|_{H^{1}}^{2} & \leqslant\left(\lambda_{v}-\lambda_{u}\right) \int_{\Omega} z_{v}\left(z_{v}-u\right)+\int_{\Omega}\left(f\left(u^{2}\right)-f\left(v^{2}\right)\right) z_{v}\left(z_{v}-u\right) \\
& =\frac{1}{2}\left(\lambda_{v}-\lambda_{u}\right)\left\|z_{v}-u\right\|_{L^{2}}^{2}+\int_{\Omega}\left(f\left(u^{2}\right)-f\left(v^{2}\right)\right) u\left(z_{v}-u\right)+\int_{\Omega}\left(f\left(u^{2}\right)-f\left(v^{2}\right)\right)\left(z_{v}-u\right)^{2} .
\end{aligned}
$$

To conclude the argument, we need again to distinguish the two cases $X=H_{\#}^{1}(\Omega)$ and $X=H_{0}^{1}(\Omega)$. In the former case, we can use (3.5), (3.8), (3.13) and (3.15) to get

$$
\begin{aligned}
\gamma\left\|z_{v}-u\right\|_{H^{1}}^{2} & \leqslant C\left(1+\|v\|_{L^{6}}^{2 q}\right)\left(\left\|z_{v}-u\right\|_{L^{2}}^{2}+\left\|z_{v}-u\right\|_{L^{6}}\right)\|u-v\|_{L^{\max (r, 2)}} \\
& +C\|u-v\|_{L^{2}}\left\|z_{v}-u\right\|_{L^{3}}\left\|z_{v}-u\right\|_{L^{6}} \\
& \leqslant C\left(1+\|v\|_{L^{6}}^{2 q}\right)\left\|z_{v}-u\right\|_{H^{1}}\|u-v\|_{L^{\max (r, 2)}}
\end{aligned}
$$

where we have used that $\left\|z_{v}-u\right\|_{L^{2}} \leqslant\left\|z_{v}\right\|_{L^{2}}+\|u\|_{L^{2}}=2$. In the latter case, from (3.5), (3.9) and (3.16) we have

$$
\begin{aligned}
\gamma\left\|z_{v}-u\right\|_{H^{1}}^{2} & \leqslant \frac{1}{2}\left(2 \varepsilon+C_{\varepsilon}\left(1+\|v\|_{L^{6}}^{2 q}\right)\|u-v\|_{L^{\max (r, 2)}}\right)\left\|z_{v}-u\right\|_{L^{2}}^{2} \\
& +C\left(1+\|v\|_{L^{6}}^{2 q}\right)\left\|z_{v}-u\right\|_{L^{6}}\|u-v\|_{L^{r}} \\
& +\varepsilon\left\|z_{v}-u\right\|_{L^{2}}^{2}+C_{\varepsilon}\|u-v\|_{L^{2}}\left\|z_{v}-u\right\|_{L^{3}}\left\|z_{v}-u\right\|_{L^{6}} .
\end{aligned}
$$

We can choose $\varepsilon=\gamma / 4$ and get (3.14). This completes the proof.

Proof of Lemma 3.5. We first notice that if $\lambda_{2, v} \geqslant \lambda_{2, u}$, then

$$
\lambda_{2, v}-\lambda_{v} \geqslant g+\lambda-\lambda_{v}
$$

so that $\lambda_{2, v}-\lambda_{v} \geqslant g / 2$ follows from (3.15) and (3.16) provided $\|u-v\|_{H^{1}}$ being small enough.

Let us now deal with the case where $\lambda_{2, v}<\lambda_{2, u}$. Since

$$
\lambda_{2, u}=\left\langle A_{u} z_{2, u}, z_{2, u}\right\rangle \leqslant C
$$

we have

$$
\forall v \in X, \quad \lambda_{2, v}<C .
$$

On the other hand, using again (3.3), we get

$$
\forall v \in X, \quad \lambda_{2, v}=\left\langle A_{v} z_{2, v}, z_{2, v}\right\rangle \geqslant \frac{\alpha}{2}\left\|z_{2, v}\right\|_{H^{1}}^{2}-\frac{\alpha}{2}-\beta_{0} .
$$

Hence, there exists a constant $C \in \mathbb{R}_{+}$such that

$$
\forall v \in X, \quad\left\|z_{2, v}\right\|_{H^{1}} \leqslant C .
$$


We now decompose $z_{2, v}$ as $z_{2, v}=\left(u, z_{2, v}\right)_{L^{2}} u+\alpha_{2, v} z_{2, v}^{\perp}$ with $\alpha_{2, v} \geqslant 0$ and $z_{2, v}^{\perp} \in u^{\perp}$ such that $\left\|z_{2, v}^{\perp}\right\|_{L^{2}}=1$. We have

$$
\alpha_{2, v}^{2}=1-\left(u, z_{2, v}\right)_{L^{2}}^{2}=1-\left(u-z_{v}, z_{2, v}\right)_{L^{2}}^{2} \geqslant 1-\left\|u-z_{v}\right\|_{L^{2}}^{2} .
$$

We deduce from (3.14) that there exists $0<\eta_{0} \leqslant 1$ such that

$$
\forall v \in \mathscr{B}_{u, \eta_{0}}, \quad\left\|u-z_{v}\right\|_{H^{1}} \leqslant 1 / 2,
$$

where $\mathscr{B}_{u, \eta_{0}}$ is the ball in $H^{1}$ with center $u$ and radius $\eta_{0}$. It then follows from (3.34) that

$$
\forall v \in \mathscr{B}_{u, \eta_{0}}, \quad \frac{1}{\alpha_{2, v}^{2}} \leqslant 1+2\left\|u-z_{v}\right\|_{L^{2}}^{2} .
$$

As $\lambda_{2, u}$ is the smallest eigenvalue of $A_{u}$ in $u^{\perp}$, we obtain, using again (3.34) and the above estimate,

$$
\begin{aligned}
\forall v \in \mathscr{B}_{u, \eta_{0}}, \quad \lambda_{2, u} & \leqslant\left\langle A_{u} z_{2, v}^{\perp}, z_{2, v}^{\perp}\right\rangle \\
& =\frac{1}{\alpha_{2, v}^{2}}\left\langle A_{u}\left(z_{2, v}-\left(u, z_{2, v}\right)_{L^{2}} u\right), z_{2, v}-\left(u, z_{2, v}\right)_{L^{2}} u\right\rangle \\
& =\frac{1}{\alpha_{2, v}^{2}}\left(\left\langle A_{u} z_{2, v}, z_{2, v}\right\rangle-\lambda\left(u, z_{2, v}\right)_{L^{2}}^{2}\right) \\
& \leqslant \frac{1}{\alpha_{2, v}^{2}}\left(\left\langle A_{u} z_{2, v}, z_{2, v}\right\rangle+|\lambda|\left\|u-z_{v}\right\|_{L^{2}}^{2}\right) \\
& =\frac{1}{\alpha_{2, v}^{2}}\left(\left\langle A_{v} z_{2, v}, z_{2, v}\right\rangle+\int_{\Omega}\left(f\left(u^{2}\right)-f\left(v^{2}\right)\right) z_{2, v}^{2}+|\lambda|\left\|u-z_{v}\right\|_{L^{2}}^{2}\right) \\
& \leqslant\left(1+2\left\|u-z_{v}\right\|_{L^{2}}^{2}\right)\left(\lambda_{2, v}+|\lambda|\left\|u-z_{v}\right\|_{L^{2}}^{2}+\int_{\Omega}\left(f\left(u^{2}\right)-f\left(v^{2}\right)\right) z_{2, v}^{2}\right),
\end{aligned}
$$

hence

$\lambda_{2, v}-\lambda_{2, u} \geqslant-\left(\lambda_{v}-\lambda\right)-2 \lambda_{2, v}\left\|u-z_{v}\right\|_{L^{2}}^{2}-\left(1+2\left\|u-z_{v}\right\|_{L^{2}}^{2}\right)\left(|\lambda|\left\|u-z_{v}\right\|_{L^{2}}^{2}+\int_{\Omega}\left(f\left(u^{2}\right)-f\left(v^{2}\right)\right) z_{2, v}^{2}\right)$.

Therefore, for any $v \in \mathscr{B}_{u, \eta_{0}}$, we have

$$
\begin{aligned}
\lambda_{2, v}-\lambda_{v} \geqslant & g-\left(\lambda_{v}-\lambda\right)-2 \lambda_{2, v}\left\|u-z_{v}\right\|_{L^{2}}^{2} \\
& -\left(1+2\left\|u-z_{v}\right\|_{L^{2}}^{2}\right)\left(|\lambda|\left\|u-z_{v}\right\|_{L^{2}}^{2}+\int_{\Omega}\left(f\left(u^{2}\right)-f\left(v^{2}\right)\right) z_{2, v}^{2}\right) .
\end{aligned}
$$

The existence of some $0<\eta \leqslant \eta_{0}$ such that $\lambda_{2, v}-\lambda_{v} \geqslant g / 2$ for all $v \in \mathscr{B}_{u, \eta}$ easily follows from (3.14), (3.15), (3.16), (3.33) and (3.35) also in the case where $\lambda_{2, v}<\lambda_{2, u}$.

Proof of Proposition 3.1. Let $0<\eta \leqslant 1$ be as in Lemma 3.5, $v \in X$ such that $\|u-v\|_{H^{1}} \leqslant \eta$, and $w \in X$ such that $\|w\|_{L^{2}}=1$ and $\left(w, z_{v}\right)_{L^{2}} \geqslant 0$. Note that $\|v\|_{H^{1}} \leqslant\|u\|_{H^{1}}+1$. Using (3.3), (3.13) and the fact that $f$ is non-negative on $\mathbb{R}_{+}$, we have

$$
\begin{aligned}
\forall z \in X, \quad\left\langle\left(A_{v}-\lambda_{v}\right) z, z\right\rangle_{X^{\prime}, X} & =a(z, z)+\int_{\Omega} f\left(v^{2}\right) z^{2}-\lambda_{v}\|z\|_{L^{2}}^{2} \\
& \geqslant \frac{\alpha}{2}\|\nabla z\|_{L^{2}}^{2}-\left(\lambda_{v}+\beta_{0}\right)\|z\|_{L^{2}}^{2} \\
& \geqslant \frac{\alpha}{2}\|\nabla z\|_{L^{2}}^{2}-\beta\|z\|_{L^{2}}^{2},
\end{aligned}
$$


where the constant $\beta$ is independent of $v$ and $z$. In particular,

$$
\left\langle\left(A_{v}-\lambda_{v}\right)\left(w-z_{v}\right),\left(w-z_{v}\right)\right\rangle_{X^{\prime}, X} \geqslant \frac{\alpha}{2}\left\|\nabla\left(w-z_{v}\right)\right\|_{L^{2}}^{2}-\beta\left\|w-z_{v}\right\|_{L^{2}}^{2} .
$$

From Lemma 3.5, we see that for all $z \in X$ such that $\left(z, z_{v}\right) \geqslant 0$,

$$
\left\langle\left(A_{v}-\lambda_{v}\right) z, z\right\rangle_{X^{\prime}, X} \geqslant \frac{g}{2}\left\|z-\left(z_{v}, z\right)_{L^{2}} z_{v}\right\|_{L^{2}}^{2}=\frac{g}{2}\left(\|z\|_{L^{2}}^{2}-\left|\left(z_{v}, z\right)_{L^{2}}\right|^{2}\right) .
$$

Therefore, we have

$$
\begin{aligned}
\left\langle\left(A_{v}-\lambda_{v}\right)\left(w-z_{v}\right),\left(w-z_{v}\right)\right\rangle_{X^{\prime}, X} & \geqslant \frac{g}{2}\left(\left\|w-z_{v}\right\|_{L^{2}}^{2}-\left|\left(z_{v}, w-z_{v}\right)\right|^{2}\right) \\
& \geqslant \frac{g}{2}\left(\left\|w-z_{v}\right\|_{L^{2}}^{2}-\left(1-\left(z_{v}, w\right)\right)\right) \\
& =\frac{g}{4}\left\|w-z_{v}\right\|_{L^{2}}^{2} .
\end{aligned}
$$

Combining (3.36) and (3.37) provides the lower bound of (3.17). We get the upper bound from the following estimate

$$
\begin{aligned}
\left\langle\left(A_{v}-\lambda_{v}\right)\left(w-z_{v}\right),\left(w-z_{v}\right)\right\rangle_{X^{\prime}, X} & =a\left(w-z_{v}, w-z_{v}\right)+\int_{\Omega} f\left(v^{2}\right)\left(w-z_{v}\right)^{2}-\lambda_{v} \int_{\Omega}\left(w-z_{v}\right)^{2} \\
& \leqslant C\left\|w-z_{v}\right\|_{H^{1}}^{2},
\end{aligned}
$$

where we have used (3.3), (3.4) and (3.13).

\subsection{Basic error analysis of scheme 1}

LEMMA 3.6 Let $u_{\delta_{\mathrm{f}}}^{\delta_{\mathrm{c}}}$ be a solution of (2.1). Under assumptions (1.2)-(1.6), we have

$$
\lim _{0<\delta_{\mathrm{f}} \leqslant \delta_{\mathrm{c}} \rightarrow 0}\left\|u-u_{\delta_{\mathrm{f}}}^{\delta_{\mathrm{c}}}\right\|_{H^{1}}=0
$$

Proof. In this proof, $C$ and $C_{\varepsilon}$ are constants independent of $\delta_{\mathrm{c}}$ and $\delta_{\mathrm{f}}$. We first notice that

$$
\left\|u-u_{\delta_{\mathrm{f}}}^{\delta_{\mathrm{c}}}\right\|_{H^{1}} \leqslant\left\|u-u_{0}^{\delta_{\mathrm{c}}}\right\|_{H^{1}}+\left\|u_{0}^{\delta_{\mathrm{c}}}-u_{\delta_{\mathrm{f}}}^{\delta_{\mathrm{c}}}\right\|_{H^{1}}
$$

We know from (1.11) that $\left\|u_{\delta_{\mathrm{c}}}\right\|_{H^{1}} \leqslant\|u\|_{H^{1}}+1$ for all $\delta_{\mathrm{c}}>0$ small enough. Using (3.14) with $v=u_{\delta_{\mathrm{c}}}$ (so that $\lambda_{v}=\lambda_{0}^{\delta_{\mathrm{c}}}$ and $z_{v}=u_{0}^{\delta_{\mathrm{c}}}$ ), we obtain

$$
\left\|u-u_{0}^{\delta_{\mathrm{c}}}\right\|_{H^{1}} \leqslant C\left\|u-u_{\delta_{\mathrm{c}}}\right\|_{H^{1}},
$$

which together with (1.11) with $\delta=\delta_{\mathrm{c}}$ implies

$$
\lim _{\delta_{\mathrm{c}} \rightarrow 0}\left\|u-u_{0}^{\delta_{\mathrm{c}}}\right\|_{H^{1}}=0
$$

For each $\delta_{\mathrm{f}}>0$, let $\Pi_{\delta_{\mathrm{f}}}: X \rightarrow X_{\delta_{\mathrm{f}}}$ be the orthogonal projection on $X_{\delta_{\mathrm{f}}}$ for the $H^{1}$-scalar product: for any $w \in X$,

$$
\left\|w-\Pi_{\delta_{\mathrm{f}}} w\right\|_{H^{1}}=\min _{v_{\delta_{\mathrm{f}}} \in X_{\delta_{\mathrm{f}}}}\left\|w-v_{\delta_{\mathrm{f}}}\right\|_{H^{1}}
$$


Again from (1.11), for any $\eta>0$, there exists $\delta_{\mathrm{c}}^{0}>0$ such that for all $0<\delta_{\mathrm{c}} \leqslant \delta_{\mathrm{c}}^{0},\left\|u-u_{\delta_{\mathrm{c}}}\right\|_{H^{1}} \leqslant \eta$. Assuming that $\left(u_{\delta_{\mathrm{f}}}^{\delta_{\mathrm{c}}}, u_{0}^{\delta_{\mathrm{c}}}\right){ }_{L^{2}} \geqslant 0$, we deduce from Proposition 3.1 that for all $0<\delta_{\mathrm{f}} \leqslant \delta_{\mathrm{c}} \leqslant \delta_{\mathrm{c}}^{0}$,

$$
\begin{aligned}
\left\|u_{\delta_{\mathrm{f}}}^{\delta_{\mathrm{c}}}-u_{0}^{\delta_{\mathrm{c}}}\right\|_{H^{1}}^{2} & \leqslant c_{0}^{-1}\left\langle\left(A_{u_{\delta_{\mathrm{c}}}}-\lambda_{0}^{\delta_{\mathrm{c}}}\right)\left(u_{\delta_{\mathrm{f}}}^{\delta_{\mathrm{c}}}-u_{0}^{\delta_{\mathrm{c}}}\right),\left(u_{\delta_{\mathrm{f}}}^{\delta_{\mathrm{c}}}-u_{0}^{\delta_{\mathrm{c}}}\right)\right\rangle_{X^{\prime}, X} \\
& =c_{0}^{-1}\left(\left\langle A_{u_{\delta_{\mathrm{c}}}} u_{\delta_{\mathrm{f}}}^{\delta_{\mathrm{f}}}, u_{\delta_{\mathrm{f}}}^{\delta_{\mathrm{f}}}\right\rangle_{X^{\prime}, X}-\left\langle A_{u_{\delta_{\mathrm{c}}}} u_{0}^{\delta_{\mathrm{c}}}, u_{0}^{\delta_{\mathrm{c}}}\right\rangle_{X^{\prime}, X}\right) \\
& \leqslant c_{0}^{-1}\left(\left\langle A_{u_{\delta_{\mathrm{c}}}} \frac{\Pi_{\delta_{\mathrm{f}}} u_{0}^{\delta_{\mathrm{c}}}}{\left\|\Pi_{\delta_{\mathrm{f}}} u_{0}^{\delta_{\mathrm{c}}}\right\|_{L^{2}}}, \frac{\Pi_{\delta_{\mathrm{f}}} u_{0}^{\delta_{\mathrm{c}}}}{\left\|\Pi_{\delta_{\mathrm{f}}} u_{0}^{\delta_{\mathrm{c}}}\right\|_{L^{2}}}\right\rangle_{X^{\prime}, X}-\left\langle A_{u_{\delta_{\mathrm{c}}}} u_{0}^{\delta_{\mathrm{c}}}, u_{0}^{\delta_{\mathrm{c}}}\right\rangle_{X^{\prime}, X}\right) \\
& =c_{0}^{-1}\left\langle\left(A_{u_{\delta_{\mathrm{c}}}}-\lambda_{0}^{\delta_{\mathrm{c}}}\right)\left(\frac{\Pi_{\delta_{\mathrm{f}}} u_{0}^{\delta_{\mathrm{c}}}}{\left\|\Pi_{\delta_{\mathrm{f}}} u_{0}^{\delta_{\mathrm{c}}}\right\|_{L^{2}}}-u_{0}^{\delta_{\mathrm{c}}}\right),\left(\frac{\Pi_{\delta_{\mathrm{f}}} u_{0}^{\delta_{\mathrm{c}}}}{\left\|\Pi_{\delta_{\mathrm{f}}} u_{0}^{\delta_{\mathrm{c}}}\right\|_{L^{2}}}-u_{0}^{\delta_{\mathrm{c}}}\right)\right\rangle_{X^{\prime}, X} \\
& \leqslant c_{0}^{-1} C_{0}\left\|\frac{\Pi_{\delta_{\mathrm{f}}} u_{0}^{\delta_{\mathrm{c}}}}{\left\|\Pi_{\delta_{\mathrm{f}}} u_{0}^{\delta_{\mathrm{c}}}\right\|_{L^{2}}}-u_{0}^{\delta_{\mathrm{c}}}\right\|_{H^{1}}^{2} \\
& \leqslant c_{0}^{-1} C_{0}\left(1+\frac{\left\|\Pi_{\delta_{\mathrm{f}}} u_{0}^{\delta_{\mathrm{c}}}\right\|_{H^{1}}}{\left\|\Pi_{\delta_{\mathrm{f}}} u_{0}^{\delta_{\mathrm{c}}}\right\|_{L^{2}}}\right)^{2}\left\|\Pi_{\delta_{\mathrm{f}}} u_{0}^{\delta_{\mathrm{c}}}-u_{0}^{\delta_{\mathrm{c}}}\right\|_{H^{1}}^{2} \\
& \leqslant c_{0}^{-1} C_{0}\left(1+\frac{\left\|u_{0}^{\delta_{\mathrm{c}}}\right\|_{H^{1}}}{\left\|\Pi_{\delta_{\mathrm{f}}} u_{0}^{\delta_{\mathrm{c}}}\right\|_{L^{2}}}\right)^{2}\left\|\Pi_{\delta_{\mathrm{f}}} u_{0}^{\delta_{\mathrm{c}}}-u_{0}^{\delta_{\mathrm{c}}}\right\|_{H^{1}}^{2} .
\end{aligned}
$$

Since $\lim _{\delta_{\mathrm{f}} \rightarrow 0}\left\|u_{0}^{\delta_{\mathrm{c}}}-\Pi_{\delta_{\mathrm{f}}} u_{0}^{\delta_{\mathrm{c}}}\right\|_{L^{2}}=0$ and $\left\|u_{0}^{\delta_{\mathrm{c}}}\right\|_{L^{2}}=1$, there exists $\delta_{\mathrm{f}}^{0}>0$ such that

$$
\forall 0<\delta_{\mathrm{f}} \leqslant \delta_{\mathrm{f}}^{0}, \quad\left\|\Pi_{\delta_{\mathrm{f}}}{u_{0}}_{\mathrm{c}}\right\|_{L^{2}} \geqslant \frac{1}{2}
$$

It follows that for $0<\delta_{\mathrm{f}} \leqslant \delta_{\mathrm{c}} \leqslant \delta_{\mathrm{f}}^{0}$,

$$
\lim _{\delta_{\mathrm{f}} \rightarrow 0}\left\|u_{\delta_{\mathrm{f}}}^{\delta_{\mathrm{c}}}-u_{0}^{\delta_{\mathrm{c}}}\right\|_{H^{1}}=0
$$

which, together with (3.38) and (3.39), leads to the desired result.

LEMMA 3.7 Let $P_{\delta_{\mathrm{f}}}: u^{\perp} \rightarrow u^{\perp} \cap X_{\delta_{\mathrm{f}}}$ be the projection operator defined by

$$
\forall w_{\delta_{\mathrm{f}}} \in u^{\perp} \cap X_{\delta_{\mathrm{f}}}, \forall v \in u^{\perp}, \quad\left(v-P_{\delta_{\mathrm{f}}} v, w_{\delta_{\mathrm{f}}}\right)_{H^{1}}=0 .
$$

We have

$$
\left\|v-P_{\delta_{\mathrm{f}}} v\right\|_{H^{1}} \leqslant C \min _{v_{\delta_{\mathrm{f}}} \in X_{\delta_{\mathrm{f}}}}\left\|v-v_{\delta_{\mathrm{f}}}\right\|_{H^{1}} .
$$

Proof. For any $v \in u^{\perp}$, we have

$$
\left(\Pi_{\delta_{\mathrm{f}}} v-\frac{\left(\Pi_{\delta_{\mathrm{f}}} v, u\right)_{L^{2}} \Pi_{\delta_{\mathrm{f}}} u}{\left(\Pi_{\delta_{\mathrm{f}}} u, u\right)_{L^{2}}}\right) \in u^{\perp} \cap X_{\delta_{\mathrm{f}}}
$$


so that

$$
\begin{aligned}
\left\|v-P_{\delta_{\mathrm{f}}} v\right\|_{H^{1}} & \leqslant\left\|v-\Pi_{\delta_{\mathrm{f}}} v+\frac{\left(\Pi_{\delta_{\mathrm{f}}} v, u\right)_{L^{2}} \Pi_{\delta_{\mathrm{f}}} u}{\left(\Pi_{\delta_{\mathrm{f}}} u, u\right)_{L^{2}}}\right\|_{H^{1}} \\
& \leqslant\left\|v-\Pi_{\delta_{\mathrm{f}}} v\right\|_{H^{1}}+\left\|\frac{\left(\Pi_{\delta_{\mathrm{f}}} v-v, u\right)_{L^{2}} \Pi_{\delta_{\mathrm{f}}} u}{\left(\Pi_{\delta_{\mathrm{f}}} u, u\right)_{L^{2}}}\right\|_{H^{1}} \\
& \leqslant\left(1+\frac{\left\|\Pi_{\delta_{\mathrm{f}}} u\right\|_{H^{1}}}{\left(\Pi_{\delta_{\mathrm{f}}} u, u\right)_{L^{2}}}\right)\left\|v-\Pi_{\delta_{\mathrm{f}}} v\right\|_{H^{1}} \\
& \leqslant C \min _{v_{\delta_{\mathrm{f}}} \in X_{\delta_{\mathrm{f}}}}\left\|v_{\delta_{\mathrm{f}}}-v\right\|_{H^{1}} .
\end{aligned}
$$

This completes the proof.

In order to state the main result of this section, we need to introduce the following object: for all $v \in L^{2}(\Omega)$, we denote by $\psi_{v} \in u^{\perp}$ the unique solution to the adjoint problem: find $\psi_{v} \in u^{\perp}$ such that

$$
\forall w \in u^{\perp}, \quad\left\langle\left(A_{u}-\lambda\right) \psi_{v}, w\right\rangle_{X^{\prime}, X}=(v, w)_{L^{2}} .
$$

The existence and uniqueness of the solution to (3.41) is a straightforward consequence of (3.11) and Lax-Milgram lemma. It follows from (3.11) that

$$
\forall v \in L^{2}(\Omega), \quad\left\|\psi_{v}\right\|_{H^{1}} \leqslant M_{2}^{-1}\|v\|_{X^{\prime}} \leqslant M_{2}^{-1}\|v\|_{L^{2}} .
$$

THEOREM 3.2 Under assumptions (1.2)-(1.6), there exist $\delta_{1}>0$ and $C \in \mathbb{R}_{+}$such that for all $0<\delta_{\mathrm{f}} \leqslant$ $\delta_{\mathrm{c}} \leqslant \delta_{1}$,

$$
\frac{\gamma}{2}\left\|u-u_{\delta_{\mathrm{f}}}^{\delta_{\mathrm{c}}}\right\|_{H^{1}}^{2} \leqslant E\left(u_{\delta_{\mathrm{f}}}^{\delta_{\mathrm{c}}}\right)-E(u) \leqslant C\left\|u-u_{\delta_{\mathrm{f}}}^{\delta_{\mathrm{f}}}\right\|_{H^{1}}^{2}
$$

and for all $r$ such that $\frac{6}{5} \leqslant r=\frac{6}{5-2 q}<6$ :

$$
\begin{gathered}
\left\|u-u_{\delta_{\mathrm{f}}}^{\delta_{\mathrm{f}}}\right\|_{H^{1}} \leqslant C\left(\min _{v_{\delta_{\mathrm{f}}} \in X_{\delta_{\mathrm{f}}}}\left\|u-v_{\delta_{\mathrm{f}}}\right\|_{H^{1}}+\left\|u-u_{\delta_{\mathrm{c}}}\right\|_{L^{r}}+\left\|u-u_{\delta_{\mathrm{f}}}^{\delta_{\mathrm{f}}}\right\|_{L^{r}}\right), \\
\left|\lambda-\lambda_{\delta_{\mathrm{f}}}^{\delta_{\mathrm{c}}}\right| \leqslant C\left(\left\|u-u_{\delta_{\mathrm{f}}}^{\delta_{\mathrm{c}}}\right\|_{H^{1}}^{2}+\left\|u-u_{\delta_{\mathrm{c}}}\right\|_{L^{r}}+\left\|u-u_{\delta_{\mathrm{f}}}^{\delta_{\mathrm{c}}}\right\|_{L^{r}}\right), \\
\left\|u-u_{\delta_{\mathrm{f}}}^{\delta_{\mathrm{f}}}\right\|_{L^{2}}^{2}=\int_{\Omega}\left(f\left(u_{\delta_{\mathrm{c}}}^{2}\right)-f\left(u^{2}\right)\right) u_{\delta_{\mathrm{f}}}^{\delta_{\mathrm{f}}} P_{\delta_{\mathrm{f}}} \psi+\left(\lambda-\lambda_{\delta_{\mathrm{f}}}^{\delta_{\mathrm{c}}}\right) \int_{\Omega}\left(u_{\delta_{\mathrm{f}}}^{\delta_{\mathrm{c}}}-u\right) P_{\delta_{\mathrm{f}}} \psi \\
+\left\langle\left(A_{u}-\lambda\right)\left(\psi-P_{\delta_{\mathrm{f}}} \psi\right),\left(u-u_{\delta_{\mathrm{f}}}^{\delta_{\mathrm{c}}}\right)\right\rangle_{X^{\prime}, X}+\frac{1}{4}\left\|u-u_{\delta_{\mathrm{f}}}^{\delta_{\mathrm{f}}}\right\|_{L^{2}}^{4},
\end{gathered}
$$

where we have set $\psi=\psi_{u-u_{\delta_{\mathrm{f}}}^{\delta_{\mathrm{c}}}}$.

Proof. Let us recall that for all $w \in X$ such that $\|w\|_{L^{2}}=1$,

$$
\begin{aligned}
E(w)-E(u)= & \frac{1}{2}\left\langle\left(A_{u}-\lambda\right)(w-u),(w-u)\right\rangle_{X^{\prime}, X} \\
& +\frac{1}{2} \int_{\Omega}\left(F\left(w^{2}\right)-F\left(u^{2}\right)-f\left(u^{2}\right)\left(w^{2}-u^{2}\right)\right) .
\end{aligned}
$$


This equality, referred to as (32) in Cancès et al. (2010), can be derived easily. Using (3.12) and the convexity of $F$, we obtain, for $w=u_{\delta_{\mathrm{f}}}^{\delta_{\mathrm{c}}}$,

$$
E\left(u_{\delta_{\mathrm{f}}}^{\delta_{\mathrm{c}}}\right)-E(u) \geqslant \frac{\gamma}{2}\left\|u-u_{\delta_{\mathrm{f}}}^{\delta_{\mathrm{c}}}\right\|_{H^{1}}^{2},
$$

that is the lower bound in (3.42). We now observe that

$$
\begin{aligned}
F\left(w^{2}\right)-F\left(u^{2}\right)-f\left(u^{2}\right)\left(w^{2}-u^{2}\right) & =\left(w^{2}-u^{2}\right) \int_{0}^{1}\left(f\left(u^{2}+t\left(w^{2}-u^{2}\right)\right)-f\left(u^{2}\right)\right) d t \\
& =\left(w^{2}-u^{2}\right)^{2} \int_{0}^{1}\left(\int_{0}^{t} f^{\prime}\left(u^{2}+s\left(w^{2}-u^{2}\right)\right) d s\right) d t \\
& =\left(w^{2}-u^{2}\right)^{2} \int_{0}^{1}(1-s) f^{\prime}\left(u^{2}+s\left(w^{2}-u^{2}\right)\right) d s .
\end{aligned}
$$

We are led to split the domain $\Omega$ into four parts

$$
\begin{gathered}
\Omega_{1}=\left\{x \in \Omega, u(x)<\frac{|w(x)|}{2}\right\}, \quad \Omega_{2}=\left\{x \in \Omega, \frac{|w(x)|}{2} \leqslant u(x)<|w(x)|\right\}, \\
\Omega_{3}=\{x \in \Omega,|w(x)| \leqslant u(x)<2|w(x)|\} \quad \text { and } \quad \Omega_{4}=\{x \in \Omega, u(x) \geqslant 2|w(x)|\},
\end{gathered}
$$

where we remark that, over $\Omega_{2} \cup \Omega_{3},|w(x)| \leqslant 2\|u\|_{L^{\infty}}$. Hence, from the assumption (1.6) made on $F^{\prime \prime}=f^{\prime}$, we deduce that $\left(u^{2}+s\left(w^{2}-u^{2}\right)\right)\left(f^{\prime}\left(u^{2}+s\left(w^{2}-u^{2}\right)\right)\right)$ is bounded over $\Omega_{2} \cup \Omega_{3}$ by a constant (say $C_{3}$ ).

We infer from (3.10), (3.46) and (3.47) that

$$
\begin{aligned}
E(w)-E(u) \leqslant & \frac{M_{1}}{2}\|w-u\|_{H^{1}}^{2}+\frac{C_{3}}{2} \int_{\Omega_{2} \cup \Omega_{3}}\left(w^{2}-u^{2}\right)^{2}\left(\int_{0}^{1} \frac{1-s}{u^{2}+s\left(w^{2}-u^{2}\right)} d s\right) \\
& +\frac{1}{2} \int_{\Omega_{1} \cup \Omega_{4}}\left(w^{2}-u^{2}\right)\left(\int_{0}^{1}\left(f\left(u^{2}+t\left(w^{2}-u^{2}\right)\right)-f\left(u^{2}\right)\right) d t\right) \\
\leqslant & \frac{M_{1}}{2}\|w-u\|_{H^{1}}^{2}+\frac{C_{3}}{2} \int_{\Omega_{2}}\left(u^{2}-w^{2}-w^{2} \ln \left(\frac{u^{2}}{w^{2}}\right)\right)+\frac{C_{3}}{2} \int_{\Omega_{3}}\left(u^{2}-w^{2}+w^{2} \ln \left(\frac{w^{2}}{u^{2}}\right)\right) \\
& +\frac{1}{2} \int_{\Omega_{1}}\left(u^{2}-w^{2}\right)\left(\int_{0}^{1}\left(f\left(u^{2}\right)-f\left(u^{2}+t\left(w^{2}-u^{2}\right)\right)\right) d t\right) \\
& +\frac{1}{2} \int_{\Omega_{4}}\left(w^{2}-u^{2}\right)\left(\int_{0}^{1}\left(f\left(u^{2}+t\left(w^{2}-u^{2}\right)\right)-f\left(u^{2}\right)\right) d t\right) .
\end{aligned}
$$

Using that $-\ln (1-a) \leqslant a+2 a^{2}$, for any $a, 0 \leqslant a \leqslant 3 / 4$, we first get that, on $\Omega_{2}$,

$$
\left(u^{2}-w^{2}\right)-w^{2} \ln \left(\frac{u^{2}}{w^{2}}\right)=\left(u^{2}-w^{2}\right)-w^{2} \ln \left(1-\frac{w^{2}-u^{2}}{w^{2}}\right) \leqslant 2 \frac{\left(w^{2}-u^{2}\right)^{2}}{w^{2}} \leqslant 8(|w|-u)^{2} \leqslant 8(w-u)^{2} .
$$

Then, using that $\ln (1-a) \leqslant-a$ and $-\ln (1-a) \leqslant 4 a$, for any $a, 0 \leqslant a \leqslant 3 / 4$, we get that, on $\Omega_{3}$,

$$
\begin{aligned}
u^{2}-w^{2}+w^{2} \ln \left(\frac{w^{2}}{u^{2}}\right) & =u^{2}-w^{2}+u^{2} \ln \left(1-\frac{u^{2}-w^{2}}{u^{2}}\right)+\left(w^{2}-u^{2}\right) \ln \left(1-\frac{u^{2}-w^{2}}{u^{2}}\right) \\
& \leqslant 4 \frac{\left(w^{2}-u^{2}\right)^{2}}{u^{2}} \leqslant 16(|w|-u)^{2} \leqslant 16(w-u)^{2} .
\end{aligned}
$$


Finally, the facts that $f$ is positive, increasing, and that $0 \leqslant\left|w^{2}-u^{2}\right| \leqslant 3(|w|-u)^{2} \leqslant 3(w-u)^{2}$ on $\Omega_{1} \cup \Omega_{4}$, we get

$$
\begin{aligned}
E(w)-E(u) & \leqslant \frac{M_{1}}{2}\|w-u\|_{H^{1}}^{2}+8 C_{3} \int_{\Omega_{2} \cup \Omega_{3}}(u-w)^{2}+3 \int_{\Omega_{1}} f\left(u^{2}\right)(w-u)^{2}+3 \int_{\Omega_{4}} f\left(w^{2}\right)(w-u)^{2} \\
& \leqslant \frac{M_{1}}{2}\|w-u\|_{H^{1}}^{2}+8 C_{3} \int_{\Omega_{2} \cup \Omega_{3}}(u-w)^{2}+3 \int_{\Omega_{1}} f\left(u^{2}\right)(w-u)^{2}+C \int_{\Omega_{4}}\left(1+|w|^{2 q}\right)(w-u)^{2} .
\end{aligned}
$$

Taking $w=u_{\delta_{\mathrm{f}}}^{\delta_{\mathrm{c}}}$, we obtain

$$
E\left(u_{\delta_{\mathrm{f}}}^{\delta_{\mathrm{c}}}\right)-E(u) \leqslant M_{2}\left\|u-u_{\delta_{\mathrm{f}}}^{\delta_{\mathrm{c}}}\right\|_{H^{1}}^{2},
$$

where the constant $M_{2}$ depends on the $H^{1}$-norm of $u_{\delta_{\mathrm{f}}}^{\delta_{\mathrm{c}}}$, which is itself uniformly bounded when $0<$ $\delta_{\mathrm{f}} \leqslant \delta_{\mathrm{c}} \leqslant \delta_{1}$. The proof of (3.42) is complete.

Recall that (see (33) in Cancès et al. (2010))

$$
\lambda_{\delta_{\mathrm{f}}}^{\delta_{\mathrm{c}}}-\lambda=\left\langle\left(A_{u}-\lambda\right)\left(u-u_{\delta_{\mathrm{f}}}^{\delta_{\mathrm{c}}}\right),\left(u-u_{\delta_{\mathrm{f}}}^{\delta_{\mathrm{c}}}\right)\right\rangle_{X^{\prime}, X}+\int_{\Omega}\left(f\left(u_{\delta_{\mathrm{c}}}^{2}\right)-f\left(u^{2}\right)\right)\left(u_{\delta_{\mathrm{f}}}^{\delta_{\mathrm{c}}}\right)^{2} .
$$

Using on the one hand (3.4) with $w=u_{\delta_{\mathrm{f}}}^{\delta_{\mathrm{c}}}+u_{\delta_{\mathrm{c}}}, z=u_{\delta_{\mathrm{f}}}^{\delta_{\mathrm{c}}}-u_{\delta_{\mathrm{c}}}$ and both $v=u$, and $v=u_{\delta_{\mathrm{c}}}$, and on the other hand (3.6) with $v=u_{\delta_{\mathrm{c}}}$, we get

$$
\begin{aligned}
\int_{\Omega}\left(f\left(u_{\delta_{\mathrm{c}}}^{2}\right)-f\left(u^{2}\right)\right)\left(u_{\delta_{\mathrm{f}}}^{\delta_{\mathrm{c}}}\right)^{2} & =\int_{\Omega}\left(f\left(u_{\delta_{\mathrm{c}}}^{2}\right)-f\left(u^{2}\right)\right)\left(\left(u_{\delta_{\mathrm{f}}}^{\delta_{\mathrm{c}}}\right)^{2}-u_{\delta_{\mathrm{c}}}^{2}\right)+\int_{\Omega}\left(f\left(u_{\delta_{\mathrm{c}}}^{2}\right)-f\left(u^{2}\right)\right) u_{\delta_{\mathrm{c}}}^{2} \\
& \leqslant C\left(\left\|u-u_{\delta_{\mathrm{c}}}\right\|_{L^{r}}+\left\|u-u_{\delta_{\mathrm{f}}}^{\delta_{\mathrm{c}}}\right\|_{L^{r}}\right) .
\end{aligned}
$$

Therefore, we obtain that for all $0<\delta_{\mathrm{f}} \leqslant \delta_{\mathrm{c}} \leqslant \delta_{1}$,

$$
\left|\lambda_{\delta_{\mathrm{f}}}^{\delta_{\mathrm{c}}}-\lambda\right| \leqslant C\left(\left\|u-u_{\delta_{\mathrm{f}}}^{\delta_{\mathrm{c}}}\right\|_{H^{1}}^{2}+\left\|u-u_{\delta_{\mathrm{c}}}\right\|_{L^{r}}+\left\|u-u_{\delta_{\mathrm{f}}}^{\delta_{\mathrm{c}}}\right\|_{L^{r}}\right) .
$$

Let us now estimate $\left\|u-u_{\delta_{\mathrm{f}}}^{\delta_{\mathrm{f}}}\right\|_{H^{1}}$. We have

$$
\left\|u-u_{\delta_{\mathrm{f}}}^{\delta_{\mathrm{f}}}\right\|_{H^{1}} \leqslant\left\|u-u_{\delta_{\mathrm{f}}}^{\delta_{\mathrm{c}}}\right\|_{H^{1}}+\left\|u_{\delta_{\mathrm{f}}}^{\delta_{\mathrm{f}}}-u_{\delta_{\mathrm{f}}}^{\delta_{\mathrm{c}}}\right\|_{H^{1}}
$$

where $u_{\delta_{\mathrm{f}}}^{\delta_{\mathrm{c}} *}$ is defined by

$$
u_{\delta_{\mathrm{f}}}^{\delta_{\mathrm{c}} *}=u_{\delta_{\mathrm{f}}}^{\delta_{\mathrm{c}}}+\left(1-\int_{\Omega} u u_{\delta_{\mathrm{f}}}^{\delta_{\mathrm{c}}}\right) u
$$

It is easy to see that $v:=u-u_{\delta_{\mathrm{f}}}^{\delta_{\mathrm{c}} *} \in u^{\perp}$ and

$$
u_{\delta_{\mathrm{f}}}^{\delta_{\mathrm{c}}}-u_{\delta_{\mathrm{f}}}^{\delta_{\mathrm{c}}}=\frac{1}{2} u\left\|u-u_{\delta_{\mathrm{f}}}^{\delta_{\mathrm{c}}}\right\|_{L^{2}}^{2}
$$

We then only need to estimate $\left\|u-u_{\delta_{\mathrm{f}}}^{\delta_{\mathrm{c}} *}\right\|_{H^{1}}$. With the previous notation, we have

$$
\left\|v-\Pi_{\delta_{\mathrm{f}}} v\right\|_{H^{1}} \leqslant\|v\|_{H^{1}}
$$


and

$$
\begin{aligned}
v-\Pi_{\delta_{\mathrm{f}}} v & =u-u_{\delta_{\mathrm{f}}}^{\delta_{\mathrm{c}}}-u+u \int_{\Omega} u u_{\delta_{\mathrm{f}}}^{\delta_{\mathrm{c}}}-\Pi_{\delta_{\mathrm{f}}}\left(u-u_{\delta_{\mathrm{f}}}^{\delta_{\mathrm{c}}}-u+u \int_{\Omega} u u_{\delta_{\mathrm{f}}}^{\delta_{\mathrm{c}}}\right) \\
& =-u_{\delta_{\mathrm{f}}}^{\delta_{\mathrm{c}}}+u \int_{\Omega} u u_{\delta_{\mathrm{f}}}^{\delta_{\mathrm{c}}}-\Pi_{\delta_{\mathrm{f}}}\left(-u_{\delta_{\mathrm{f}}}^{\delta_{\mathrm{c}_{\mathrm{c}}}}+u \int_{\Omega} u u_{\delta_{\mathrm{f}}}^{\delta_{\mathrm{c}}}\right) \\
& =-u_{\delta_{\mathrm{f}}}^{\delta_{\mathrm{c}}}+u \int_{\Omega} u u_{\delta_{\mathrm{f}}}^{\delta_{\mathrm{c}}}+u_{\delta_{\mathrm{f}}}^{\delta_{\mathrm{c}}}-\Pi_{\delta_{\mathrm{f}}} u \int_{\Omega} u u_{\delta_{\mathrm{f}}}^{\delta_{\mathrm{c}}} \\
& =\left(u-\Pi_{\delta_{\mathrm{f}}} u\right) \int_{\Omega} u u_{\delta_{\mathrm{f}}}^{\delta_{\mathrm{c}}}
\end{aligned}
$$

Hence,

$$
\left\|v-\Pi_{\delta_{\mathrm{f}}} v\right\|_{H^{1}} \leqslant c\left\|u-\Pi_{\delta_{\mathrm{f}}} u\right\|_{H^{1}} .
$$

Due to (3.11) and (3.52), we have

$$
\begin{aligned}
M_{2}\|v\|_{H^{1}}^{2} \leqslant & \left\langle\left(A_{u}-\lambda\right) v, v\right\rangle_{X^{\prime}, X} \\
= & \left\langle\left(A_{u}-\lambda\right)\left(v-\Pi_{\delta_{\mathrm{f}}} v\right), v\right\rangle_{X^{\prime}, X}+\left\langle\left(A_{u}-\lambda\right) \Pi_{\delta_{\mathrm{f}}} v, u_{\delta_{\mathrm{f}}}^{\delta_{\mathrm{f}}}-u_{\delta_{\mathrm{f}}}^{\delta_{\mathrm{f}_{\mathrm{f}} *}}\right\rangle_{X^{\prime}, X} \\
& +\left\langle\left(A_{u}-\lambda\right) \Pi_{\delta_{\mathrm{f}}} v, u-u_{\delta_{\mathrm{f}}}^{\delta_{\mathrm{f}}}\right\rangle_{X^{\prime}, X} \\
= & \left\langle\left(A_{u}-\lambda\right)\left(v-\Pi_{\delta_{\mathrm{f}}} v\right), v\right\rangle_{X^{\prime}, X}-\frac{1}{2}\left\|u-u_{\delta_{\mathrm{f}}}^{\delta_{\mathrm{f}}}\right\|_{L^{2}}^{2}\left\langle\left(A_{u}-\lambda\right) \Pi_{\delta_{\mathrm{f}}} v, u\right\rangle_{X^{\prime}, X} \\
& +\left\langle\left(A_{u}-\lambda\right) \Pi_{\delta_{\mathrm{f}}} v, u-u_{\delta_{\mathrm{f}}}^{\delta_{\mathrm{f}}}\right\rangle_{X^{\prime}, X} .
\end{aligned}
$$

For any $v_{\delta_{\mathrm{f}}} \in X_{\delta_{\mathrm{f}}}$, we have

$$
\begin{aligned}
\left\langle\left(A_{u}-\lambda\right)\left(u-u_{\delta_{\mathrm{f}}}^{\delta_{\mathrm{c}}}\right), v_{\delta_{\mathrm{f}}}\right\rangle_{X^{\prime}, X}= & -\left\langle\left(A_{u}-\lambda\right) u_{\delta_{\mathrm{f}}}^{\delta_{\mathrm{c}}}, v_{\delta_{\mathrm{f}}}\right\rangle_{X^{\prime}, X} \\
= & \int_{\Omega}\left(f\left(u_{\delta_{\mathrm{c}}}^{2}\right)-f\left(u^{2}\right)\right) u_{\delta_{\mathrm{f}}}^{\delta_{\mathrm{c}}} v_{\delta_{\mathrm{f}}}-\left\langle\left(A_{u_{\delta_{\mathrm{c}}}}-\lambda\right) u_{\delta_{\mathrm{f}}}^{\delta_{\mathrm{f}}}, v_{\delta_{\mathrm{f}}}\right\rangle_{X^{\prime}, X} \\
= & \int_{\Omega}\left(f\left(u_{\delta_{\mathrm{c}}}^{2}\right)-f\left(u^{2}\right)\right) u_{\delta_{\mathrm{f}}}^{\delta_{\mathrm{c}}} v_{\delta_{\mathrm{f}}}+\left(\lambda-\lambda_{\delta_{\mathrm{f}}}^{\delta_{\mathrm{c}}}\right) \int_{\Omega} u_{\delta_{\mathrm{f}}}^{\delta_{\mathrm{c}}} v_{\delta_{\mathrm{f}}} \\
= & \int_{\Omega}\left(f\left(u_{\delta_{\mathrm{c}}}^{2}\right)-f\left(u^{2}\right)\right)\left(u_{\delta_{\mathrm{f}}}^{\delta_{\mathrm{c}}}-u\right) v_{\delta_{\mathrm{f}}}+\int_{\Omega}\left(f\left(u_{\delta_{\mathrm{c}}}^{2}\right)-f\left(u^{2}\right)\right) u v_{\delta_{\mathrm{f}}} \\
& +\left(\lambda-\lambda \delta_{\delta_{\mathrm{f}}}^{\delta_{\mathrm{f}}}\right) \int_{\Omega} u_{\delta_{\mathrm{f}}}^{\delta_{\mathrm{c}}} v_{\delta_{\mathrm{f}}},
\end{aligned}
$$

which together with (3.4) and (3.5) implies that for any $v_{\delta_{\mathrm{f}}} \in X_{\delta_{\mathrm{f}}}$,

$$
\left\langle\left(A_{u}-\lambda\right)\left(u-u_{\delta_{\mathrm{f}}}^{\delta_{\mathrm{c}}}\right), v_{\delta_{\mathrm{f}}}\right\rangle_{X^{\prime}, X} \leqslant C\left(\left\|u-u_{\delta_{\mathrm{f}}}^{\delta_{\mathrm{c}}}\right\|_{L^{r}}+\left\|u-u_{\delta_{\mathrm{c}}}\right\|_{L^{r}}\right)\left\|v_{\delta_{\mathrm{f}}}\right\|_{H^{1}}+\left|\lambda-\lambda_{\delta_{\mathrm{f}}}^{\delta_{\mathrm{c}}}\right| .
$$

From (3.54) and (3.56), we have

$$
\|v\|_{H^{1}} \leqslant C\left(\left\|v-\Pi_{\delta_{\mathrm{f}}} v\right\|_{H^{1}}+\frac{1}{2}\left\|u-u_{\delta_{\mathrm{f}}}^{\delta_{\mathrm{c}}}\right\|_{L^{2}}^{2}+\left\|u-u_{\delta_{\mathrm{f}}}^{\delta_{\mathrm{c}}}\right\|_{L^{r}}+\left\|u-u_{\delta_{\mathrm{c}}}\right\|_{L^{r}}+\left|\lambda-\lambda_{\delta_{\mathrm{f}}}^{\delta_{\mathrm{c}}}\right|\right) .
$$

Therefore, for all $0<\delta_{\mathrm{f}} \leqslant \delta_{\mathrm{c}} \leqslant \delta_{1}$, we get from (3.49), (3.50), (3.52), (3.53) and (3.57) that

$$
\left\|u-u_{\delta_{\mathrm{f}}}^{\delta_{\mathrm{c}}}\right\|_{H^{1}} \leqslant C\left(\left\|u-\Pi_{\delta_{\mathrm{f}}} u\right\|_{H^{1}}+\left\|u-u_{\delta_{\mathrm{f}}}^{\delta_{\mathrm{c}}}\right\|_{L^{r}}+\left\|u-u_{\delta_{\mathrm{c}}}\right\|_{L^{r}}\right) .
$$


Thus (3.43) is proved. Let us now consider the $L^{2}$ estimate and set $\psi=\psi_{u-u_{\delta_{\mathrm{f}}}^{\delta_{\mathrm{c}}}}$ (see (3.41)). From (3.41) and (3.52), there holds

$$
\begin{aligned}
\left\|u-u_{\delta_{\mathrm{f}}}^{\delta_{\mathrm{f}}}\right\|_{L^{2}}^{2} & =\int_{\Omega}\left(u-u_{\delta_{\mathrm{f}}}^{\delta_{\mathrm{c}}}\right)\left(u-u_{\delta_{\mathrm{f}}}^{\delta_{\mathrm{f}^{*}}}\right)+\int_{\Omega}\left(u-u_{\delta_{\mathrm{f}}}^{\delta_{\mathrm{c}}}\right)\left(u_{\delta_{\mathrm{f}}}^{\delta_{\mathrm{c}} *}-u_{\delta_{\mathrm{f}}}^{\delta_{\mathrm{c}}}\right) \\
& =\int_{\Omega}\left(u-u_{\delta_{\mathrm{f}}}^{\delta_{\mathrm{c}}}\right)\left(u-u_{\delta_{\mathrm{f}}}^{\delta_{\mathrm{f}^{*}}}\right)+\frac{1}{2}\left\|u-u_{\delta_{\mathrm{f}}}^{\delta_{\mathrm{f}}}\right\|_{L^{2}}^{2} \int_{\Omega} u\left(u-u_{\delta_{\mathrm{f}}}^{\delta_{\mathrm{c}}}\right) \\
& =\int_{\Omega}\left(u-u_{\delta_{\mathrm{f}}}^{\delta_{\mathrm{c}}}\right)\left(u-u_{\delta_{\mathrm{f}}}^{\delta_{\mathrm{c}} *}\right)+\frac{1}{4}\left\|u-u_{\delta_{\mathrm{f}}}^{\delta_{\mathrm{f}_{\mathrm{f}}}}\right\|_{L^{2}}^{4} \\
& =\left\langle\left(A_{u}-\lambda\right) \psi,\left(u-u_{\delta_{\mathrm{f}}}^{\delta_{\mathrm{c}} *}\right)\right\rangle_{X^{\prime}, X}+\frac{1}{4}\left\|u-u_{\delta_{\mathrm{f}}}^{\delta_{\mathrm{c}}}\right\|_{L^{2}}^{4} .
\end{aligned}
$$

We have

$$
\begin{aligned}
\left\langle\left(A_{u}-\lambda\right) \psi,\left(u-u_{\delta_{\mathrm{f}}}^{\delta_{\mathrm{f}}}\right)\right\rangle_{X^{\prime}, X} & =\left\langle\left(A_{u}-\lambda\right) \psi,\left(u-u_{\delta_{\mathrm{f}}}^{\delta_{\mathrm{c}}}\right)\right\rangle_{X^{\prime}, X}-\left(1-\int_{\Omega} u u_{\delta_{\mathrm{f}}}^{\delta_{\mathrm{c}}}\right)\left\langle\left(A_{u}-\lambda\right) \psi, u\right\rangle_{X^{\prime}, X} \\
& =\left\langle\left(A_{u}-\lambda\right) \psi,\left(u-u_{\delta_{\mathrm{f}}}^{\delta_{\mathrm{c}}}\right)\right\rangle_{X^{\prime}, X},
\end{aligned}
$$

which, together with (3.55), (3.58), and the fact that $P_{\delta_{\mathrm{f}}} \psi \in u^{\perp}$ yields

$$
\begin{aligned}
\left\|u-u_{\delta_{\mathrm{f}}}^{\delta_{\mathrm{f}}}\right\|_{L^{2}}^{2}= & \left\langle\left(A_{u}-\lambda\right) \psi, u-u_{\delta_{\mathrm{f}}}^{\delta_{\mathrm{c}}}\right\rangle_{X^{\prime}, X}+\frac{1}{4}\left\|u-u_{\delta_{\mathrm{f}}}^{\delta_{\mathrm{c}}}\right\|_{L^{2}}^{4} \\
= & \left\langle\left(A_{u}-\lambda\right)\left(u-u_{\delta_{\mathrm{f}}}^{\delta_{\mathrm{f}}}\right), P_{\delta_{\mathrm{f}}} \psi\right\rangle_{X^{\prime}, X}+\left\langle\left(A_{u}-\lambda\right)\left(\psi-P_{\delta_{\mathrm{f}}} \psi\right),\left(u-u_{\delta_{\mathrm{f}}}^{\delta_{\mathrm{c}}}\right)\right\rangle_{X^{\prime}, X} \\
& +\frac{1}{4}\left\|u-u_{\delta_{\mathrm{f}}}^{\delta_{\mathrm{f}}}\right\|_{L^{2}}^{4} \\
= & \int_{\Omega}\left(f\left(u_{\delta_{\mathrm{c}}}^{2}\right)-f\left(u^{2}\right)\right) u_{\delta_{\mathrm{f}}}^{\delta_{\mathrm{f}}} P_{\delta_{\mathrm{f}}} \psi+\left(\lambda-\lambda_{\delta_{\mathrm{f}}}^{\delta_{\mathrm{f}}}\right) \int_{\Omega}\left(u_{\delta_{\mathrm{f}}}^{\delta_{\mathrm{f}}}-u\right) P_{\delta_{\mathrm{f}}} \psi \\
& +\left\langle\left(A_{u}-\lambda\right)\left(\psi-P_{\delta_{\mathrm{f}}} \psi\right),\left(u-u_{\delta_{\mathrm{f}}}^{\delta_{\mathrm{f}}}\right)\right\rangle_{X^{\prime}, X}+\frac{1}{4}\left\|u-u_{\delta_{\mathrm{f}}}^{\delta_{\mathrm{f}}}\right\|_{L^{2}}^{4},
\end{aligned}
$$

which proves (3.45).

\section{Spectral Fourier discretization}

In this section, we consider $\Omega=(0,2 \pi)^{d}$ with $d=1,2,3$ and $X=H_{\#}^{1}(\Omega)$, and we make the following assumptions:

$$
\begin{aligned}
& \qquad V \in H_{\#}^{\sigma}(\Omega) \text { for some } \sigma>d / 2 \\
& \text { the function } F \text { satisfies (1.4)-(1.6) and is in } C^{[\sigma]+2, \sigma-[\sigma]+\varepsilon}((0,+\infty), \mathbb{R}) \text {. }
\end{aligned}
$$

The positive solution u to (1.1), which satisfies the elliptic equation

$$
-\Delta u+V u+f\left(u^{2}\right) u=\lambda u,
$$

then is in $H_{\#}^{\sigma+2}(\Omega)$ and is bounded away from 0 .

A natural discretization of (1.1) consists in using a Fourier basis. Denoting for any $k \in \mathbb{Z}^{d}$ by $e_{k}(x)=(2 \pi)^{-d / 2} e^{i k \cdot x}$, we have for all $v \in L^{2}(\Omega)$,

$$
v(x)=\sum_{k \in \mathbb{Z}^{d}} \hat{v}_{k} e_{k}(x),
$$


where $\hat{v}_{k}$ is the $k$ th Fourier coefficient of $v$ :

$$
\hat{v}_{k}:=\int_{\Omega} v(x) \overline{e_{k}(x)} d x=(2 \pi)^{-d / 2} \int_{\Omega} v(x) e^{-i k \cdot x} d x
$$

The Fourier spectral approximation of the solution to (1.1) is based on the choice

$$
X_{M}=\left\{\sum_{k \in \mathbb{Z}^{d},|k| * \leqslant M} c_{k} e_{k}, \forall k, \overline{c_{k}}=c_{-k}\right\},
$$

where $|k|_{*}$ denotes either the $l^{2}$-norm or the $l^{\infty}$-norm of the wave vector $k$.

Endowing $H_{\#}^{\rho}(\Omega)$ with the norm defined by

$$
\|v\|_{H^{\rho}}=\left(\sum_{k \in \mathbb{Z}^{d}}\left(1+|k|_{*}^{2}\right)^{\rho}\left|\hat{v}_{k}\right|^{2}\right)^{1 / 2},
$$

we obtain that for all $\tau \in \mathbb{R}$, and all $v \in H_{\#}^{\tau}(\Omega)$, the best approximation of $v$ in $H_{\#}^{\rho}(\Omega)$ for any $\rho \leqslant \tau$ is

$$
\Pi_{M} v=\sum_{k \in \mathbb{Z}^{d},|k| * \leqslant M} \hat{v}_{k} e_{k}
$$

For all real numbers $\rho$ and $\tau$ with $\rho \leqslant \tau$, we have

$$
\forall v \in H_{\#}^{\tau}, \quad\left\|v-\Pi_{M} v\right\|_{H^{\rho}} \leqslant \frac{1}{M^{\tau-\rho}}\|v\|_{H^{\tau}} .
$$

In this section, we take $\delta_{\mathrm{c}}=M^{-1}$ and $\delta_{\mathrm{f}}=N^{-1}(M \leqslant N)$, and $u_{\delta_{\mathrm{c}}}, u_{0}^{\delta_{\mathrm{c}}}$ and $u_{\delta_{\mathrm{f}}}^{\delta_{\mathrm{c}}}$ are denoted as $u_{M}$, $u_{0}^{M}$ and $u_{N}^{M}$, respectively. It is easy to see that $u_{0}^{M} \in H_{\#}^{\sigma+2}(\Omega)$. Aligning the functions $u_{M}, u_{0}^{M}$ and $u_{N}^{M}$ in such a way that $\left(u_{0}^{M}, u_{\kappa}^{M}\right)_{L^{2}} \geqslant 0$ for $\kappa=M, N$, and using (4.3), we obtain

$$
\left\|u_{0}^{M}-\Pi_{\kappa} u_{0}^{M}\right\|_{H^{1}} \leqslant \frac{1}{\kappa^{\sigma+1}}\left\|u_{0}^{M}\right\|_{H^{\sigma+2}} .
$$

It therefore follows from Lemma 3.6 that

$$
\lim _{0<M \leqslant N \rightarrow \infty}\left\|u-u_{N}^{M}\right\|_{H^{1}}=0 .
$$

It is proved in Cancès et al. (2010) that $u_{M}$ converges to $u$ in $H_{\#}^{\sigma+2}(\Omega)$. In particular, $u / 2 \leqslant u_{M} \leqslant 2 u$ on $\Omega$ for $M$ large enough.

Besides, $u_{N}^{M}$ is solution to the elliptic equation

$$
-\Delta u_{N}^{M}+\Pi_{N}\left(V u_{N}^{M}+f\left(u_{M}^{2}\right) u_{N}^{M}\right)=\lambda_{N}^{M} u_{N}^{M} .
$$

Thus $u_{N}^{M}$ is uniformly bounded in $H_{\#}^{2}(\Omega)$, hence in $L^{\infty}(\Omega)$, and

$$
\begin{aligned}
\Delta\left(u_{N}^{M}-u\right)= & \Pi_{N}\left(V\left(u_{N}^{M}-u\right)+f\left(u_{M}^{2}\right) u_{N}^{M}-f\left(u^{2}\right) u\right) \\
& +\left(\Pi_{N}-I\right)\left(V u+f\left(u^{2}\right) u\right)-\lambda_{N}^{M}\left(u_{N}^{M}-u\right)-\left(\lambda_{N}^{M}-\lambda\right) u .
\end{aligned}
$$


Since $u_{M}$ is bounded in $L^{\infty}(\Omega), F \in C^{1}([0, \infty), \mathbb{R}) \cap C^{2}((0, \infty), \mathbb{R}), \lim _{M \rightarrow 0}\left\|u-u_{M}\right\|_{H^{1}}=0$, and $\lim _{0<M \leqslant N \rightarrow \infty} \| u-$ $u_{N}^{M} \|_{H^{1}}=0$, the right hand side of the above equality converges to 0 in $L_{\#}^{2}(\Omega)$, which implies that $\left(u_{N}^{M}\right)_{N \in \mathbb{N}}$ actually converges to $u$ in $H_{\#}^{2}(\Omega)$. With a bootstrapping argument, we also deduce from (4.4) that $u_{N}^{M}$ converges to $u$ in $H_{\#}^{\sigma+2}(\Omega)$.

Besides, the unique solution to (3.41) solves the elliptic equation

$$
-\Delta \psi_{v}+\left(V+f\left(u^{2}\right)-\lambda\right) \psi_{v}=v-(v, u)_{L^{2}} u,
$$

from which we infer that $\psi_{u-u_{N}^{M}}$ belongs to $H_{\#}^{\sigma+2}(\Omega)$ and $\left\|\psi_{u-u_{N}^{M}}\right\|_{H^{2}} \leqslant C\left\|u-u_{N}^{M}\right\|_{L^{2}}$. Hence

$$
\left\|\psi_{u-u_{N}^{M}}-\Pi_{N} \psi_{u-u_{N}^{M}}\right\|_{H^{1}} \leqslant \frac{1}{N}\left\|\psi_{u-u_{N}^{M}}\right\|_{H^{2}} \leqslant \frac{C}{N}\left\|u-u_{N}^{M}\right\|_{L^{2}}
$$

TheOREM 4.1 Under assumptions (4.1)-(4.2), there exist $C, C_{1}, C_{2} \in \mathbb{R}_{+}$such that for all $N \in \mathbb{N}$,

$$
\begin{aligned}
\left\|u-u_{M}\right\|_{H^{\tau}} & \leqslant \frac{C}{M^{\sigma+2-\tau}} \quad \text { for all }-\sigma \leqslant \tau<\sigma+2, \\
E\left(u_{N}^{M}\right)-E(u) & \leqslant C\left(\frac{1}{M^{\sigma+3}}+\frac{1}{N^{\sigma+1}}\right)^{2}, \\
\left\|u-u_{N}^{M}\right\|_{H^{1}} & \leqslant C\left(\frac{1}{M^{\sigma+3}}+\frac{1}{N^{\sigma+1}}\right), \\
\left\|u-u_{N}^{M}\right\|_{L^{2}} & \leqslant C\left(\frac{1}{M^{\min \{\sigma+4,2(\sigma+1)\}}}+\frac{1}{N}\left(\frac{1}{M^{\sigma+3}}+\frac{1}{N^{\sigma+1}}\right)\right), \\
\left|\lambda-\lambda_{N}^{M}\right| & \leqslant \frac{C_{1}}{M^{2(\sigma+1)}}+\frac{C_{2}}{N^{2(\sigma+1)}} .
\end{aligned}
$$

Proof. The proof of (4.5) is detailed in Cancès et al. (2010) $)^{2}$.

Let us first come back to (3.48), which we rewrite as,

$$
\lambda_{N}^{M}-\lambda=\left\langle\left(A_{u}-\lambda\right)\left(u-u_{N}^{M}\right), u-u_{N}^{M}\right\rangle_{X^{\prime}, X}+\int_{\Omega} w^{M, N}\left(u_{M}-u\right),
$$

with

$$
w^{M, N}=\frac{f\left(u_{M}^{2}\right)-f\left(u^{2}\right)}{u_{M}^{2}-u^{2}}\left(u_{M}+u\right)\left(u_{N}^{M}\right)^{2}=\left(u_{M}+u\right)\left(u_{N}^{M}\right)^{2} \int_{0}^{1} f^{\prime}\left(u^{2}+t\left(u_{M}^{2}-u^{2}\right)\right) d t,
$$

where the argument of $f^{\prime}$, namely $\left(u^{2}+t\left(u_{M}^{2}-u^{2}\right)\right)$, belongs to $H_{\#}^{\sigma+2}(\Omega)$ for any $t \in(0,1)$. As, for $M$ large enough, $u / 2 \leqslant u_{M} \leqslant 2 u$ on $\Omega$ for $M$, we also have $u^{2} / 4 \leqslant\left(u^{2}+t\left(u_{M}^{2}-u^{2}\right)\right) \leqslant 4 u^{2}$. As $f \in C^{[\sigma]+1, \sigma-[\sigma]+\varepsilon}\left(\left[\|u\|_{L^{\infty}}^{2} / 4,4\|u\|_{L^{\infty}}^{2}\right], \mathbb{R}\right)$, we obtain that $w^{M, N}$ is uniformly bounded in $H_{\#}^{\sigma}(\Omega)$ (at least for $N$ large enough). We therefore infer from (4.10) that, for $M$ large enough,

$$
\left|\lambda_{N}^{M}-\lambda\right| \leqslant C_{1}\left\|u-u_{M}\right\|_{H^{-\sigma}}+C_{2}\left\|u-u_{N}^{M}\right\|_{H^{1}}^{2} .
$$

${ }^{2}$ Note that, as already observed in Cancès et al. (2010), it follows from the fact that the continuous solution $u$ and the discrete ones $u_{M}$ and $u_{N}^{M}$ are bounded away from zero, the assumption that there exist $1<r \leqslant 2$ and $0 \leqslant s \leqslant 5-r$ such that

$$
\forall R>0, \exists C_{R} \in \mathbb{R}_{+} \text {s.t. } \forall 0<t_{1} \leqslant R, \forall t_{2} \in \mathbb{R},\left|F^{\prime}\left(t_{2}^{2}\right) t_{2}-F^{\prime}\left(t_{1}^{2}\right) t_{2}-2 F^{\prime \prime}\left(t_{1}^{2}\right) t_{1}^{2}\left(t_{2}-t_{1}\right)\right| \leqslant C_{R}\left(1+\left|t_{2}\right|^{s}\right)\left|t_{2}-t_{1}\right|^{r}
$$

made in Cancès et al. (2010) is actually not necessary and is thus not made here. 
We now make use of (3.45), which reads here as

$$
\begin{aligned}
\left\|u-u_{N}^{M}\right\|_{L^{2}}^{2}= & \int_{\Omega}\left(f\left(u_{M}^{2}\right)-f\left(u^{2}\right)\right) u_{N}^{M} P_{N} \psi+\left(\lambda-\lambda_{N}^{M}\right) \int_{\Omega}\left(u_{N}^{M}-u\right) P_{N} \psi \\
& +\left\langle\left(A_{u}-\lambda\right)\left(\psi-P_{N} \psi\right),\left(u-u_{N}^{M}\right)\right\rangle_{X^{\prime}, X}+\frac{1}{4}\left\|u-u_{N}^{M}\right\|_{L^{2}}^{4},
\end{aligned}
$$

with $\psi=\psi_{u-u_{N}^{M}}$. Reasoning as above, we obtain that the sequence

$$
\widetilde{w}^{M, N}=\frac{f\left(u_{M}^{2}\right)-f\left(u^{2}\right)}{u_{M}^{2}-u^{2}}\left(u_{M}+u\right) u_{N}^{M}=\left(u_{M}+u\right) u_{N}^{M} \int_{0}^{1} f^{\prime}\left(u^{2}+t\left(u_{M}^{2}-u^{2}\right)\right) d t
$$

is uniformly bounded in $H_{\#}^{\sigma}(\Omega)$ (at least for $M$ large enough). Setting $\sigma^{*}=\min \{\sigma, 2\}$, we have

$$
\begin{aligned}
\int_{\Omega}\left(f\left(u_{M}^{2}\right)-f\left(u^{2}\right)\right) u_{N}^{M} P_{N} \psi & =\int_{\Omega} \widetilde{w}^{M, N} P_{N} \psi\left(u_{M}-u\right) \\
& \leqslant\left\|u-u_{M}\right\|_{H^{-\sigma^{*}}}\left\|\widetilde{w}^{M, N} P_{N} \psi\right\|_{H^{\sigma^{*}}} \\
& \leqslant c^{\prime}\left\|u-u_{M}\right\|_{H^{-\sigma^{*}}}\left\|v_{N}\right\|_{H^{2}},
\end{aligned}
$$

which, together with (3.40), (4.3) and (4.12), implies

$$
\begin{aligned}
\left\|u-u_{N}^{M}\right\|_{L^{2}}^{2} \leqslant & C\left(\left\|u-u_{M}\right\|_{H^{-} \sigma^{*}}\left\|u-u_{N}^{M}\right\|_{L^{2}}+\left|\lambda-\lambda_{N}^{M}\right|\left\|u-u_{N}^{M}\right\|_{L^{2}}^{2}\right. \\
& \left.+\frac{1}{N}\left\|u-u_{N}^{M}\right\|_{H^{1}}\left\|u-u_{N}^{M}\right\|_{L^{2}}+\frac{1}{4}\left\|u-u_{N}^{M}\right\|_{L^{2}}^{4}\right) .
\end{aligned}
$$

Therefore, we have

$$
\left\|u-u_{N}^{M}\right\|_{L^{2}} \leqslant C\left(\frac{1}{N}\left\|u-u_{N}^{M}\right\|_{H^{1}}+\left\|u-u_{M}\right\|_{H^{-\sigma^{*}}}\right) .
$$

Let $v:=u_{N}^{M *}-u$, with $u_{N}^{M *}$ being defined as in (3.51). We deduce from (3.54) and (3.55) that

$$
\begin{aligned}
M_{2}\|v\|_{H^{1}}^{2} \leqslant & \left\langle\left(A_{u}-\lambda\right)\left(v-\Pi_{N} v\right), v\right\rangle_{X^{\prime}, X}-\frac{1}{2}\left\|u-u_{N}^{M}\right\|_{L^{2}}^{2}\left\langle\left(A_{u}-\lambda\right) \Pi_{N} v, u\right\rangle_{X^{\prime}, X} \\
& +\int_{\Omega}\left(f\left(u_{M}^{2}\right)-f\left(u^{2}\right)\right) u_{N}^{M} \Pi_{N} v+\left(\lambda-\lambda_{N}^{M}\right) \int_{\Omega} u_{N}^{M} \Pi_{N} v
\end{aligned}
$$

We also have

$$
\begin{aligned}
\int_{\Omega}\left(f\left(u_{M}^{2}\right)-f\left(u^{2}\right)\right) u_{N}^{M} \Pi_{N} v & =\int_{\Omega} \widetilde{w}^{M, N} \Pi_{N} v\left(u_{M}-u\right) \\
& \leqslant\left\|u-u_{M}\right\|_{H^{-1}}\left\|\widetilde{w}^{M, N} \Pi_{N} v\right\|_{H^{1}} \\
& \leqslant C\left\|u-u_{M}\right\|_{H^{-1}}\|v\|_{H^{1}}
\end{aligned}
$$

From (3.10), (4.14) and (4.15), we obtain

$$
M_{2}\|v\|_{H^{1}}^{2} \leqslant M_{1}\left\|v-\Pi_{N} v\right\|_{H^{1}}\|v\|_{H^{1}}+\frac{1}{2}\left\|u-u_{N}^{M}\right\|_{L^{2}}^{2}\|v\|_{H^{1}}\|u\|_{H^{1}}+\left(\left\|u-u_{M}\right\|_{H^{-1}}+\left|\lambda-\lambda_{N}^{M}\right|\right)\|v\|_{H^{1}} .
$$

Therefore,

$$
\left\|u-u_{N}^{M}\right\|_{H^{1}} \leqslant C\left(\left\|u-\Pi_{N} u\right\|_{H^{1}}+\left\|u-u_{M}\right\|_{H^{-1}}\right),
$$


which together with (4.3), (4.11) and (4.13), completes the proof of (4.6)-(4.9).

At this stage it is slightly disappointing to remark that the error on the two-grids eigenvalue has the same asymptotic behavior as the coarse approximation, nevertheless we note that the constant $C_{1}$ in front of the coarse asymptotic decay (4.9) is smaller than the one of the coarse approximation. This is very well illustrated in the numerical simulations of section 7 .

\section{Finite-element discretization}

In this section, we assume that $\Omega$ is a rectangular brick of $\mathbb{R}^{d}$ with $d=1,2,3$ and $X=H_{0}^{1}(\Omega)$.

By elliptic regularity (Gilbarg \& Trudinger (1998)), the positive solution $u$ to (1.1), which satisfies the elliptic equation

$$
-\Delta u+V u+f\left(u^{2}\right) u=\lambda u,
$$

is in $H^{2}(\Omega) \cap H_{0}^{1}(\Omega)$ whenever $V$ is in $L^{2}(\Omega)$, and is in $H^{3}(\Omega) \cap H_{0}^{1}(\Omega)$ whenever $V$ is in $H^{1}(\Omega)$ (use an extension-by-symmetry argument in order to check that there are no vertex or edge singularities, and the fact that $f^{\prime}\left(u^{2}\right) u^{2} \nabla u$ is in $L^{2}(\Omega)$ whenever $u$ is in $H^{2}(\Omega)$ ).

Considering a family of quasi-uniform triangulations $\left(\mathscr{T}_{\delta}\right)_{\delta}$ of $\Omega$, we introduce the coarse $\left(X_{H}^{p}\right)_{H}$ (associated to the triangulations indexed by $\delta=H$ ) and fine $\left(X_{h}^{\ell}\right)_{h}$ (associated to the triangulations indexed by $\delta=h$ ) finite element subspaces of $H_{0}^{1}(\Omega)$ such that :

- $X_{\delta}^{k}=\left\{v \in H_{0}^{1}(\Omega), \forall K_{\delta} \in \mathscr{T}_{\delta}, v_{\mid K_{\delta}} \in \mathbb{P}_{k}\left(K_{\delta}\right)\right\}$

- $k=p$ or $\ell(p, \ell=1$ or 2$)$

- $\delta=H$ or $h$, with $0<h \ll H$,

- $\mathscr{T}_{h}$ is a sub-triangulation of $\mathscr{T}_{H}$.

As usual, $H$ (resp. $h$ ) denote the maximum of the diameters $H_{K}, K \in \mathscr{T}_{H}$ (resp. $h_{K^{\prime}}, K^{\prime} \in \mathscr{T}_{h}$ ).

We denote by $\mathscr{I}_{\delta, k}$ the interpolation operator on $X_{\delta}^{k}$. The following estimates are classical (see e.g Bernardi et al. (2000); Ciarlet \& Lions (1991); Ern \& Guermond (2004)).

LEMMA 5.1 For any integer $n, 0 \leqslant n \leqslant k+1$, and for all $r$ and $q, 1 \leqslant r \leqslant q<+\infty$, such that $\forall K_{\delta} \in \mathscr{T}_{\delta}$, $W^{n, r}\left(K_{\delta}\right)$ is included in $C^{0}\left(K_{\delta}\right)$, there exists a positive constant $c$ depending only on $n, r$ and $q$ such that, for any function $v$ of $W^{n, r}(\Omega)$, we have :

$$
\begin{gathered}
\left\|v-\mathscr{I}_{\delta, k} v\right\|_{L^{\infty}} \leqslant c \delta^{n-\frac{d}{r}}|v|_{W^{n, r}}, \\
\left\|v-\mathscr{I}_{\delta, k} v\right\|_{W^{1, q}} \leqslant c \delta^{n-1-\frac{d}{r}+\frac{d}{q}}|v|_{W^{n, r} .}
\end{gathered}
$$

LEMmA 5.2 There exists a positive constant $c$ independent of $\delta$ such that, for any $v_{\delta} \in X_{\delta}^{k}$ we have :

$$
\left\|v_{\delta}\right\|_{L^{\infty}} \leqslant c \zeta(\delta)\left\|v_{\delta}\right\|_{H^{1}} \quad \text { where } \quad \zeta(\delta)= \begin{cases}c\left\|v_{\delta}\right\|_{H^{1}} & \text { for } \mathrm{d}=1 \\ c(1+|\log \delta|)\left\|v_{\delta}\right\|_{H^{1}} & \text { for } \mathrm{d}=2 \\ c \boldsymbol{\delta}^{-\frac{1}{2}}\left\|v_{\delta}\right\|_{H^{1}} & \text { for } \mathrm{d}=3\end{cases}
$$

Let $u_{\delta, k}$ be a solution of the minimization problem

$$
\inf \left\{E\left(v_{\delta, k}\right), v_{\delta, k} \in X_{\delta}^{k}, \int_{\Omega} v_{\delta, k}^{2}=1\right\}
$$

such that $\left(u, u_{\delta, k}\right)_{L^{2}} \geqslant 0$. Let us recall the main result in Cancès et al. (2010) concerning the finite element discretization. 
THEOREM 5.1 Assume that

$$
V \in L^{2}(\Omega), \quad \text { the function } F \text { satisfies (1.4), (1.5) for } q=1 \text {, and (1.6), }
$$

and there exist $1<r \leqslant 2$ and $0 \leqslant s+r \leqslant 3$ such that $\forall R>0, \exists C_{R} \in \mathbb{R}_{+}$for which

$$
\forall 0<t_{1} \leqslant R, \forall t_{2} \in \mathbb{R},\left|F^{\prime}\left(t_{2}^{2}\right) t_{2}-F^{\prime}\left(t_{1}^{2}\right) t_{2}-2 F^{\prime \prime}\left(t_{1}^{2}\right) t_{1}^{2}\left(t_{2}-t_{1}\right)\right| \leqslant C_{R}\left(1+\left|t_{2}\right|^{s}\right)\left|t_{2}-t_{1}\right|^{r} .
$$

Then there exist $\delta_{0}>0$ and $C \in \mathbb{R}_{+}$such that for all $0<\delta \leqslant \delta_{0}, k=1$ or $k=2$

$$
\begin{aligned}
\left\|u_{\delta, k}-u\right\|_{H^{1}} & \leqslant C \delta\|u\|_{H^{2}}, \\
\left\|u_{\delta, k}-u\right\|_{L^{2}} & \leqslant C \delta^{2}\|u\|_{H^{2}}, \\
\left\|u_{\delta, k}-u\right\|_{H^{-1}} & \leqslant C \delta^{2}\|u\|_{H^{2}}, \\
\left|\lambda_{\delta, k}-\lambda\right| & \leqslant C \delta^{2}\|u\|_{H^{2}}
\end{aligned}
$$

If, in addition,

$$
V \in H^{1}(\Omega),(5.4) \text { is satisfied for } r=2, F \in C^{3}((0,+\infty), \mathbb{R}),
$$

$$
F \in C^{3}((0,+\infty), \mathbb{R}) \text { and } F^{\prime \prime}(t) t^{1 / 2} \text { and } F^{\prime \prime \prime}(t) t^{3 / 2} \text { are locally bounded in }[0,+\infty),
$$

then there exist $\delta_{0}>0$ and $C \in \mathbb{R}_{+}$such that for all $0<\delta \leqslant \delta_{0}$,

$$
\begin{aligned}
\left\|u_{\delta, 2}-u\right\|_{H^{1}} & \leqslant C \delta^{2}\|u\|_{H^{3}}, \\
\left\|u_{\delta, 2}-u\right\|_{L^{2}} & \leqslant C \delta^{3}\|u\|_{H^{3}}, \\
\left\|u_{\delta, 2}-u\right\|_{H^{-1}} & \leqslant C \delta^{4}\|u\|_{H^{3}} \\
\left|\lambda_{\delta, 2}-\lambda\right| & \leqslant C \delta^{4}\|u\|_{H^{3}} .
\end{aligned}
$$

In this section, we will take $X_{\delta_{\mathrm{c}}}=X_{H}^{p}$, and $X_{\delta_{\mathrm{f}}}=X_{h}^{\ell}$. Let $u_{0}^{H, p} \in X$ be the unique solution of

$$
J_{H, p}=\inf \left\{E^{H, p}(v), v \in X, \int_{\Omega} v^{2}=1\right\}, \quad \text { with } \quad E^{H, p}(v)=\frac{1}{2} a(v, v)+\frac{1}{2} \int_{\Omega} f\left(u_{H, p}^{2}\right) v^{2},
$$

and $u_{\delta_{\mathrm{f}}}^{\delta_{\mathrm{c}}}=u_{h, \ell}^{H, p}$ be the solution of the following lineared eigenvalue problem (Two-grid scheme 1): find $u_{h, \ell}^{H, p} \in X_{h}^{\ell},\left\|u_{h, \ell}^{H, p}\right\|_{L^{2}}=1,\left(u, u_{h, \ell}^{H, p}\right)_{L^{2}} \geqslant 0$, and $\lambda_{h, \ell}^{H, p} \in \mathbb{R}$ such that

$$
a\left(u_{h, \ell}^{H, p}, v_{h}\right)+\int_{\Omega} f\left(u_{H, p}^{2}\right) u_{h, \ell}^{H, p} v_{h}=\lambda_{h, \ell}^{H, p} \int_{\Omega} u_{h, \ell}^{H, p} v_{h} \quad \forall v_{h} \in X_{h}^{\ell} .
$$

LEMma 5.3 If (5.3) and (5.4) are satisfied, then

$$
\lim _{H \rightarrow 0}\left\|u-u_{H, p}\right\|_{L^{\infty}}=0 \quad \text { with } p=1 \text { or } 2 .
$$

Proof. To establish this result, we first remark that

$$
\left\|u-u_{H, p}\right\|_{L^{\infty}} \leqslant\left\|u_{H, p}-I_{H, p} u\right\|_{L^{\infty}}+\left\|I_{H, p} u-u\right\|_{L^{\infty}} .
$$

From (5.1), we have

$$
\lim _{H \rightarrow 0}\left\|I_{H, p} u-u\right\|_{L^{\infty}}=0 .
$$


Using (5.2) and Lemma 5.2, we obtain

$$
\begin{aligned}
\left\|u_{H, p}-I_{H, p} u\right\|_{L^{\infty}} & \leqslant c \zeta(H)\left\|u_{H, p}-I_{H, p} u\right\|_{H^{1}} \\
& \leqslant c \zeta(H)\left(\left\|u_{H, p}-u\right\|_{H^{1}}+\left\|u-I_{H, p} u\right\|_{H^{1}}\right) \\
& \leqslant c^{\prime} \zeta(H) H^{p}\|u\|_{H^{p+1}}
\end{aligned}
$$

which implies

$$
\lim _{H \rightarrow 0}\left\|u_{H, p}-I_{H, p} u\right\|_{L^{\infty}}=0
$$

This completes the proof.

The following theorem states the behavior of the two-grid approach in the finite element context.

THEOREM 5.2 If (5.3) and (5.4) are satisfied, then there exist $C, C_{1}, C_{2} \in \mathbb{R}_{+}$and $h_{0} \in \mathbb{R}_{+}$such that for all $0<h, H \leqslant h_{0}$, we have :

$$
\begin{aligned}
E\left(u_{h, 1}^{H, 1}\right)-E(u) & \leqslant C\left(H^{2}+h\right)^{2}, \\
\left\|u-u_{h, 1}^{H, 1}\right\|_{H^{1}} & \leqslant C\left(H^{2}+h\right), \\
\left\|u-u_{h, 1}^{H, 1}\right\|_{L^{2}} & \leqslant C\left(H^{2}+h^{2}\right), \\
\left|\lambda-\lambda_{h, 1}^{H, 1}\right| & \leqslant C_{1} H^{2}+C_{1} h^{2} .
\end{aligned}
$$

If, in addition, (5.9) and (5.10) are satisfied, then there exist $c \in \mathbb{R}_{+}$and $h_{0} \in \mathbb{R}_{+}$such that for all $0<h, H \leqslant h_{0}$ and $p, \ell=1$ or 2 , we have :

$$
\begin{aligned}
& E\left(u_{h, \ell}^{H, p}\right)-E(u) \leqslant C\left(H^{p+1}+h^{\ell}\right)^{2}, \\
& \left\|u-u_{h, \ell}^{H, p}\right\|_{H^{1}} \leqslant C\left(H^{p+1}+h^{\ell}\right), \\
& \left\|u-u_{h, \ell}^{H, p}\right\|_{L^{2}} \leqslant C\left(H^{p+1}+h^{\ell+1}\right), \\
& \left|\lambda-\lambda_{h, \ell}^{H, p}\right| \leqslant C_{1} H^{2 p}+C_{2} h^{2 \ell} .
\end{aligned}
$$

Proof. We follow step by step the same lines as in Theorem 4.1. The analysis will be done gradually under the various regularity assumptions on $F$. We first start with the analysis of the eigenvalues. Proceeding as in (4.10), we get

$$
\lambda_{h, \ell}^{H, p}-\lambda=\left\langle\left(A_{u}-\lambda\right)\left(u-u_{h, \ell}^{H, p}\right), u-u_{h, \ell}^{H, p}\right\rangle_{X^{\prime}, X}+\int_{\Omega} w^{H, h}\left(u_{H, p}-u\right)
$$

with

$$
w^{H, h}=\frac{f\left(u_{H, p}^{2}\right)-f\left(u^{2}\right)}{u_{H, p}^{2}-u^{2}}\left(u_{H, p}+u\right)\left(u_{h, \ell}^{H, p}\right)^{2}=\left(u_{H, p}+u\right)\left(u_{h, \ell}^{H, p}\right)^{2} \int_{0}^{1} f^{\prime}\left(u^{2}+t\left(u_{H, p}^{2}-u^{2}\right)\right) d t .
$$

We have already derived from this equality the generic estimate (3.44), which for $q=1$ and $r=2$, gives

$$
\left|\lambda-\lambda_{h, \ell}^{H, p}\right| \leqslant C\left(\left\|u-u_{h, \ell}^{H, p}\right\|_{H^{1}}^{2}+\left\|u-u_{H, p}\right\|_{L^{2}}+\left\|u-u_{h, \ell}^{H, p}\right\|_{L^{2}}\right) .
$$

If $V$ and $F$ satisfies the additional regularity assumptions (5.9)-(5.10), then $w^{H, h}$ belongs to $H^{1}(\Omega)$ and

$$
\left|\lambda-\lambda_{h, \ell}^{H, p}\right| \leqslant C\left(\left\|u-u_{h, \ell}^{H, p}\right\|_{H^{1}}^{2}+\left\|u-u_{H, p}\right\|_{H^{-1}}\right) .
$$


We refer to the proof of Theorem 3 in Cancès et al. (2010) for details. Next for any $v_{h, \ell} \in X_{h}^{\ell}$, there holds

$$
\begin{aligned}
\int_{\Omega}\left(f\left(u_{H, p}^{2}\right)-f\left(u^{2}\right)\right) u_{h, \ell}^{H, p} v_{h, \ell} & =\int_{\Omega} w^{H, p}\left(u_{H, p}-u\right) u_{h, \ell}^{H, p} v_{h, \ell} \\
& \leqslant C\left\|w^{H, p}\right\|_{L^{6}}\left\|u_{h, \ell}^{H, p}\right\|_{L^{6}}\left\|v_{h, \ell}\right\|_{L^{6}}\left\|u_{H, p}-u\right\|_{L^{2}} \\
& \leqslant C\left\|u_{H, p}-u\right\|_{L^{2}}\left\|v_{h, \ell}\right\|_{H^{1}} .
\end{aligned}
$$

We then infer from (3.45) and (5.25) that

$$
\begin{aligned}
\left\|u_{h, \ell}^{H, p}-u\right\|_{L^{2}}^{2}= & \left(\lambda-\lambda_{h, \ell}^{H, p}\right) \int_{\Omega}\left(u_{h, \ell}^{H, p}-u\right) P_{h, \ell} \psi+\int_{\Omega}\left(f\left(u_{H, p}^{2}\right)-f\left(u^{2}\right)\right) u_{h, \ell}^{H, p} P_{h, \ell} \psi \\
+ & \left\langle\left(A_{u}-\lambda\right)\left(u-u_{h, \ell}^{H, p}\right), \psi-P_{h, \ell} \psi\right\rangle_{X^{\prime}, X}+\frac{1}{4}\left\|u-u_{h, \ell}^{H, p}\right\|_{L^{2}}^{4} \\
\leqslant & \lambda-\lambda_{h, \ell}^{H, p} \mid\left\|u-u_{h, \ell}^{H, p}\right\|_{L^{2}}^{2}+\left\|u-u_{H, p}\right\|_{L^{2}}\left\|u-u_{h, \ell}^{H, p}\right\|_{L^{2}} \\
& +h\left\|u-u_{h, \ell}^{H, p}\right\|_{H^{1}}\left\|u-u_{h, \ell}^{H, p}\right\|_{L^{2}}+\frac{1}{4}\left\|u-u_{h, \ell}^{H, p}\right\|_{L^{2}}^{4},
\end{aligned}
$$

Hence

$$
\left\|u_{h, \ell}^{H, p}-u\right\|_{L^{2}} \leqslant C\left(\left\|u-u_{H, p}\right\|_{L^{2}}+h\left\|u-u_{h, \ell}^{H, p}\right\|_{H^{1}}\right) .
$$

Inserting this result in (3.43) gives

$$
\left\|u-u_{h, \ell}^{H, p}\right\|_{H^{1}} \leqslant C\left(\left\|u-\Pi_{h, \ell} u\right\|_{H^{1}}+\left\|u-u_{H, p}\right\|_{L^{2}}\right),
$$

which leads to (5.16) and (5.20), and then to (5.15) and (5.19). Next, from (5.26), we further deduce (5.17) and (5.21). Finally, (5.18) and (5.22) are consequences of (5.23) and (5.24) respectively.

\section{The effect of numerical integration}

Let us now sketch the effect of a practical implementation of the method, and more precisely to the numerical integration of the nonlinear term. For simplicity, we focus on the case when $\mathscr{D}=I$, with periodic boundary conditions and $\Omega=[0, L)^{3}(L>0)$.

For $N_{g} \in \mathbb{N} \backslash\{0\}$, we perform the numerical integration on the cartersian grid $\mathscr{G}_{N_{g}}:=\frac{L}{N_{g}} \mathbb{Z}^{3}$. We now introduce the subspace

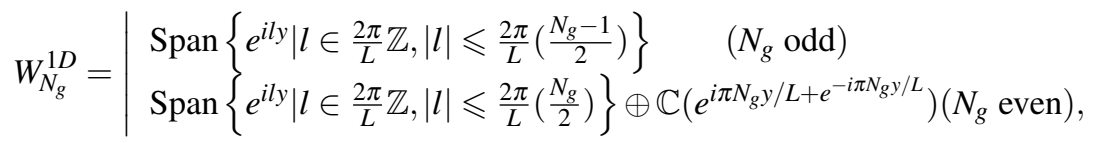

and $W_{N_{g}}^{3 D}=W_{N_{g}}^{1 D} \otimes W_{N_{g}}^{1 D} \otimes W_{N_{g}}^{1 D}$. It is then possible to define the interpolation projector $I_{N_{g}}$ from $C_{\#}^{0}(\Gamma, \mathbb{C})$ onto $W_{N_{g}}^{3 D}$ by $\left[I_{N_{g}}(\phi)\right](x)=\phi(x)$ for any $x \in \mathscr{G}_{N_{g}}$.

We now consider the following approximate problem

$$
\inf \left\{E_{N_{g}}^{M}\left(v_{N, N_{g}}\right), v_{N, N_{g}} \in X_{N}, \int_{\Omega}\left|v_{N, N_{g}}\right|^{2}=1\right\},
$$

where

$$
E_{N_{g}}^{M}\left(v_{N}\right)=\frac{1}{2} \int_{\Omega}\left|\nabla v_{N}\right|^{2}+\frac{1}{2} \int_{\Omega} I_{N_{g}}(V) v_{N}^{2}+\frac{1}{2} \int_{\Omega} f\left(u_{M}^{2}\right) v_{N}^{2} .
$$


Let us denote by $u_{N, N_{g}}^{M}$ a solution to (6.1) such that $\left(u_{N, N_{g}}^{M}, u_{N}^{M}\right) \geqslant 0$. Then $u_{N, N_{g}}^{M}$ satisfies the following Euler-Lagrange equation:

$$
\left\langle A_{u_{M}} u_{N, N_{g}}^{M}, v\right\rangle_{X^{\prime}, X}+\int_{\Omega}\left(I_{N_{g}}(V)-V\right) u_{N, N_{g}}^{M} v=\lambda_{N, N_{g}}^{M} \int_{\Omega} u_{N, N_{g}}^{M} v \quad \forall v \in X_{N} .
$$

LEMMA 6.1 There exists a positive constant $M_{3}$ such that

$$
\forall v \in X_{N} \cap\left(u_{N}^{M}\right)^{\perp}, \quad\left\langle\left(A_{u_{M}}-\lambda_{N}^{M}\right) v, v\right\rangle \geqslant M_{3}\|v\|_{H^{1}}^{2},
$$

and

$$
E^{M}\left(u_{N, N_{g}}^{M}\right)-E^{M}\left(u_{N}^{M}\right) \geqslant \frac{M_{3}}{2}\left\|u_{N, N_{g}}^{M}-u_{N}^{M}\right\|_{H^{1}}^{2},
$$

where

$$
E^{M}(v)=\frac{1}{2} \int_{\Omega}|\nabla v|^{2}+\frac{1}{2} \int_{\Omega} V v^{2}+\frac{1}{2} \int_{\Omega} f\left(u_{M}^{2}\right) v^{2}
$$

Proof. It is easy to see that

$$
E^{M}\left(u_{N, N_{g}}^{M}\right)-E^{M}\left(u_{N}^{M}\right)=\frac{1}{2}\left\langle\left(A_{u_{M}}-\lambda_{N}^{M}\right)\left(u_{N, N_{g}}^{M}-u_{N}^{M}\right), u_{N, N_{g}}^{M}-u_{N}^{M}\right\rangle_{X^{\prime}, X} .
$$

Note that $\lambda_{N}^{M}$ is the variational approximation in $X_{N}$ of some eigenvalue of $A_{u_{M}}$. As $\left(u_{M}\right)_{M \in \mathbb{N}}$ converges to $u$ in $L^{\infty}(\Omega), A_{u_{M}}-A_{u}$ converges to 0 in operator norm. So the $n^{\text {th }}$ eigenvalue of $A_{u_{M}}$ converges to the $n^{\text {th }}$ eigenvalue of $A_{u}$ when $N$ goes to infinity, the convergence being uniform in $n$. As the sequence $\left(\lambda_{N}^{M}\right)_{N \in \mathbb{N}}$ converges to $\lambda$, the non-degenerate ground state eigenvalue of $A_{u}$, we obtain that for $N$ large enough, $\lambda_{N}^{M}$ is the non-degenerate ground state eigenvalue of $A_{u_{M}}$ in $X_{N}$. We conclude the proof by proceeding as in Lemma 3.1.

Following step by step the same lines as in Cancès et al. (2010), we can prove the following result (we omit the details here for the sake brevity).

TheOREM 6.1 Assume that $V \in H_{\#}^{\sigma}(\Omega)$ for some $\sigma>d / 2$ and that the function $F$ satisfies (1.4)-(1.6) and is in $C^{[\sigma]+2, \sigma-[\sigma]+\varepsilon}((0,+\infty), \mathbb{R})$. Then there exists $C>0$ such that for all $N \in \mathbb{N}$,

$$
\begin{gathered}
E\left(u_{N, N_{g}}^{M}\right)-E(u) \leqslant C\left(\frac{1}{M^{\sigma+3}}+\frac{1}{N^{\sigma+1}}+\frac{N^{3 / 2}}{N_{g}^{\sigma}}\right)^{2}, \\
\left\|u_{N, N_{g}}^{M}-u\right\|_{H^{1}} \leqslant C\left(\frac{1}{M^{\sigma+3}}+\frac{1}{N^{\sigma+1}}+\frac{N^{3 / 2}}{N_{g}^{\sigma}}\right), \\
\left\|u_{N, N_{g}}^{M}-u\right\|_{L^{2}} \leqslant C\left(\frac{1}{M^{\sigma+3}}+\frac{1}{N^{\sigma+2}}+\frac{N^{3 / 2}}{N_{g}^{\sigma}}\right), \\
\left|\lambda_{N, N_{g}}^{M}-\lambda\right| \leqslant C\left(\frac{1}{M^{2(\sigma+1)}}+\frac{1}{N^{2(\sigma+1)}}+\frac{N^{3 / 2}}{N_{g}^{\sigma}}\right) .
\end{gathered}
$$




\section{Numerical examples}

In order to evaluate the quality of the error bounds obtained in Theorem 4.1, we have performed numerical tests with $\Omega=(0,2 \pi)$ and $f(t)=t$. The Fourier coefficients of the potential $V$ are given by

$$
\hat{V}_{k}=\frac{(-1)^{k+1}}{\sqrt{2 \pi}} \frac{1}{|k|^{2}-\frac{1}{4}},
$$

from which we deduce that $V \in H_{\#}^{\sigma}(0,2 \pi)$ for all $\sigma<3 / 2$. The reference values for $u$ and $\lambda$ are obtained for $N=500$. We first fix $M$ and study the behaviors of the numerical errors $\left\|u-u_{N}^{M}\right\|_{H^{1}},\left\|u-u_{N}^{M}\right\|_{L^{2}}$, $\left|\lambda-\lambda_{N}^{M}\right|$ and $\left|\lambda-\widetilde{\lambda}_{N}^{M}\right|$ as functions of $N$.

Let us consider for example the case when $M=10$. From the left figures of Fig. 1 and Fig. 2 we can see that $\left\|u-u_{N}^{M}\right\|_{H^{1}}$ and $\left\|u-u_{N}^{M}\right\|_{L^{2}}$ decay respectively as $N^{-2.5}$ and $N^{-3.5}$ up to $N=40$, while from $N=40$ the errors decay slowly and finally reach plateaus, on which the terms in $\frac{1}{M^{\tau}}$ dominate.

Then, we fix $N$ and study the numerical errors $\left\|u-u_{N}^{M}\right\|_{H^{1}},\left\|u-u_{N}^{M}\right\|_{L^{2}},\left|\lambda-\lambda_{N}^{M}\right|$ and $\left|\lambda-\tilde{\lambda}_{N}^{M}\right|$ as functions of $M$. From the right figures of Fig. 1 and Fig. 2 we can see that $\left\|u-u_{N}^{M}\right\|_{H^{1}}$ and $\left\|u-u_{N}^{M}\right\|_{L^{2}}$ decay respectively as $M^{-4.5}$ and $M^{-5}$ before reaching plateaus. These results are in agreement with the assertions of Theorem 4.1.

The same conclusion holds for the error on the eigenvalue, both as a function of $N$ or $M$. An interesting observation is that the two-grid scheme 1 actually leads to two approximations of the eigenvalue, the

first one being $\lambda_{\delta_{\mathrm{f}}}^{\delta_{\mathrm{c}}}$, the second being the Rayleigh quotient (2.2). Our simulations (and this can easily be confirmed by theoretical arguments) show that the rates of convergence of these two approximations are the same, and actually also similar as the plain coarse approximation (see Fig. 3 and Fig. 4), we nevertheless appreciate that the constant $\left(C_{1}\right.$ on equation 4.9$)$ in front of the coarse rate, is smaller that the associated constant (roughly equal to $C_{1}+C_{2}$ ) in the estimate of the coarse eigenvalue (see also at the end of this section). In addition, it can be noted that the accuracy is somehow better for the second approximation and that, in addition, the convergence to zero is more monotonic and smoother. We are unfortunately not able to provide an explanation of this fact.

In order to evaluate the quality of the error bounds obtained in Theorem 5.2, we have performed numerical tests, with Freefem++ Hecht (2012), with $\Omega=[0,2 \pi]^{2}, f(t)=t, V(x)=x^{2}+y^{2}$, using $\mathbb{P}_{1}$ and $\mathbb{P}_{2}$ finite elements. We denote the number of degrees of freedom in the coarse and fine grids by $\mathrm{DOF}_{H}$ and $\mathrm{DOF}_{h}$, respectively.

Fig. 5-8 show the numerical errors using $\mathbb{P}_{1}$ finite elements for both the coarse grid and the fine grid. These figures agree with the results of Theorem 5.2, except the right figure of Fig. 5 in which the term in $h$ dominates. Fig. 9-10 show the numerical errors using $\mathbb{P}_{1}$ on the coarse grid and $\mathbb{P}_{2}$ on the fine grid. Fig. 11-14 show the numerical errors using $\mathbb{P}_{2}$ finite elements on both the coarse grid and the fine grid. Similar conclusions as for the plane wave approximation hold here which illustrate the various behaviors stated in Section 5. 

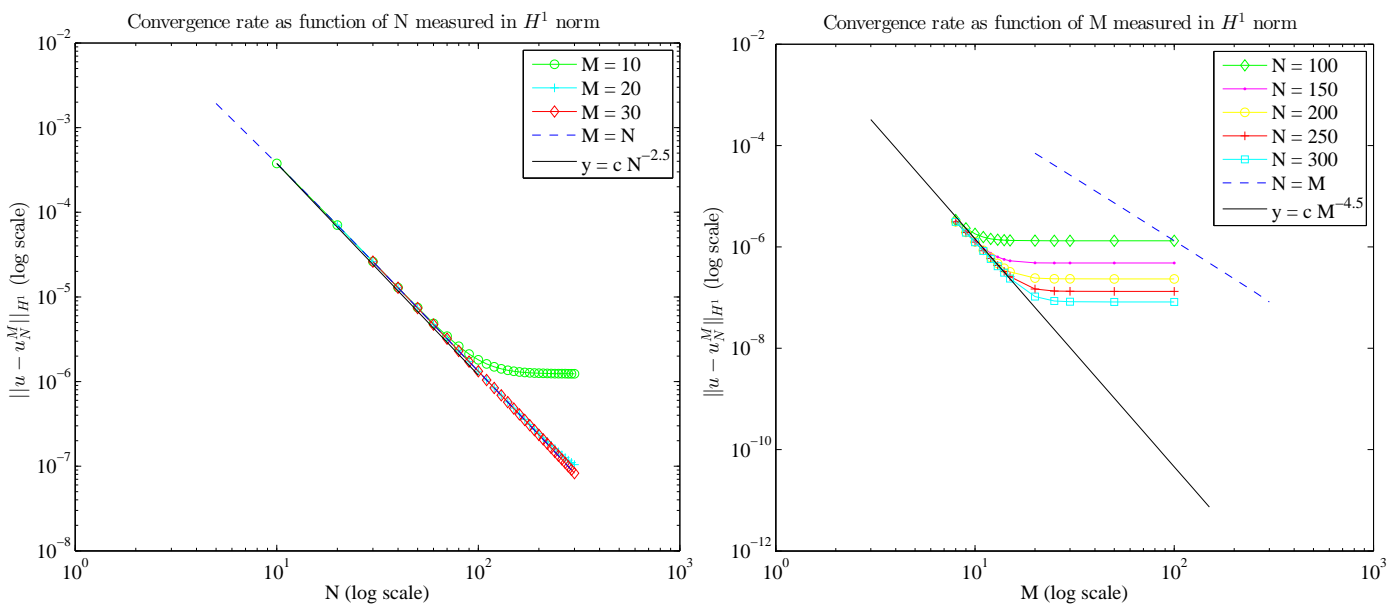

FIG. 1. Numerical errors $\left\|u-u_{N}^{M}\right\|_{H^{1}}$ (Fourier approximation), as functions of $N$ (left) and $M$ (right) (in log-log scale).
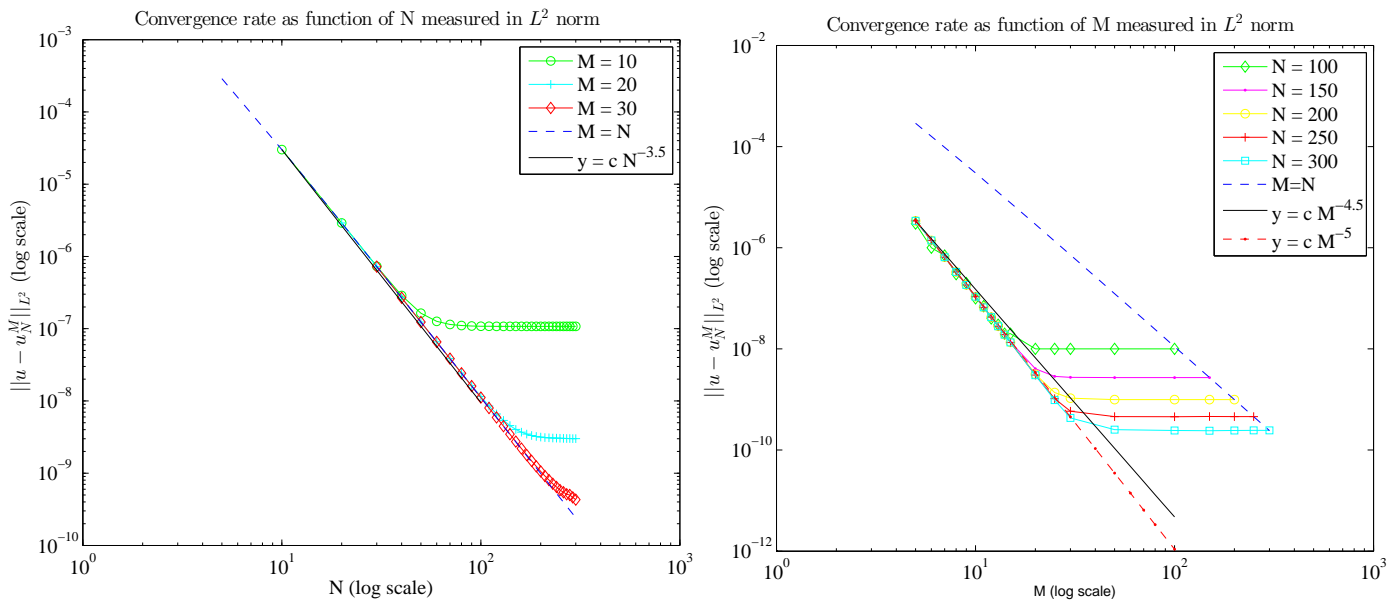

FIG. 2. Numerical errors $\left\|u-u_{N}^{M}\right\|_{L^{2}}$ (Fourier approximation), as functions of $N$ (left) and $M$ (right) (in log-log scale). 

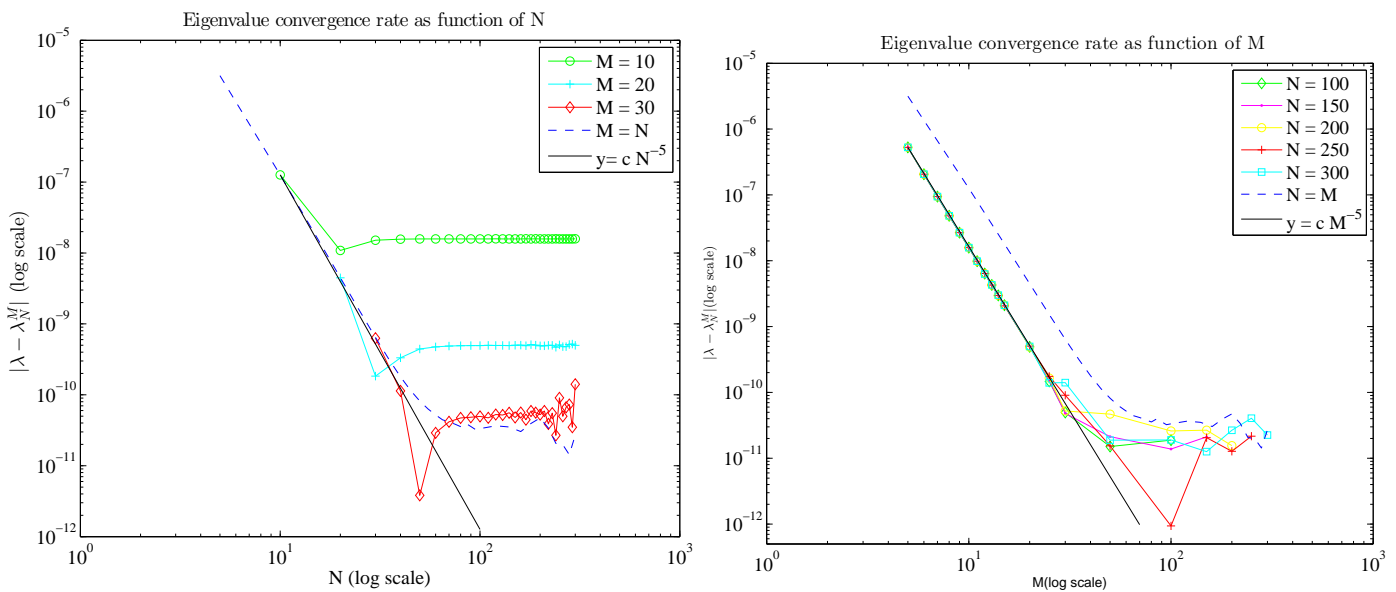

FIG. 3. Numerical errors $\left|\lambda-\lambda_{N}^{M}\right|$ (Fourier approximation), as functions of $N$ (left) and $M$ (right) (in $\log$ - $\log$ scale).
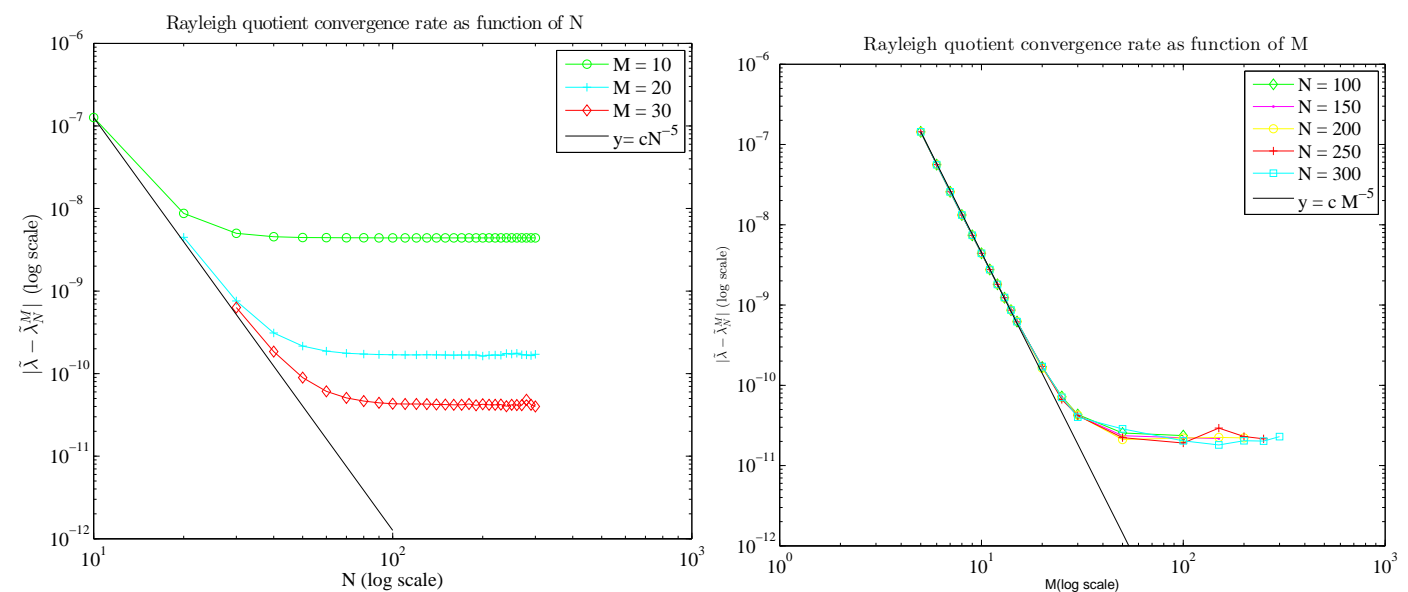

FIG. 4. Numerical errors $\left|\lambda-\tilde{\lambda}_{N}^{M}\right|$ (Fourier approximation), as functions of $N$ (left) and $M$ (right) (in log-log scale). 

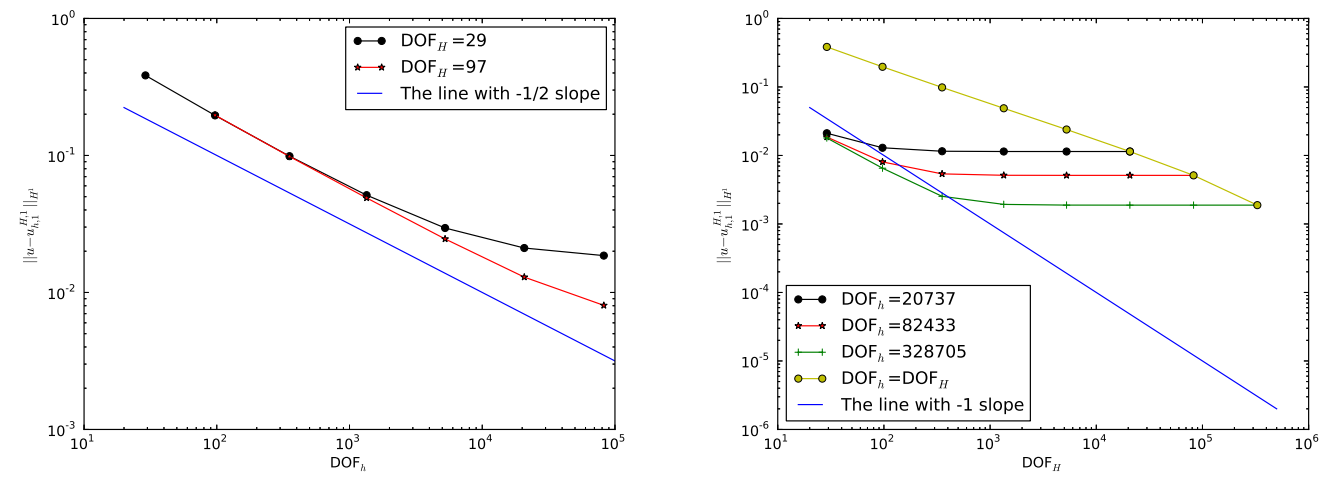

FIG. 5. Numerical errors $\left\|u-u_{h, 1}^{H, 1}\right\|_{H^{1}}\left(\mathbb{P}_{1}\right.$ finite elements), as functions of $\mathrm{DOF}_{h}$ (left) and $\mathrm{DOF}_{H}$ (right) (in log-log scale).
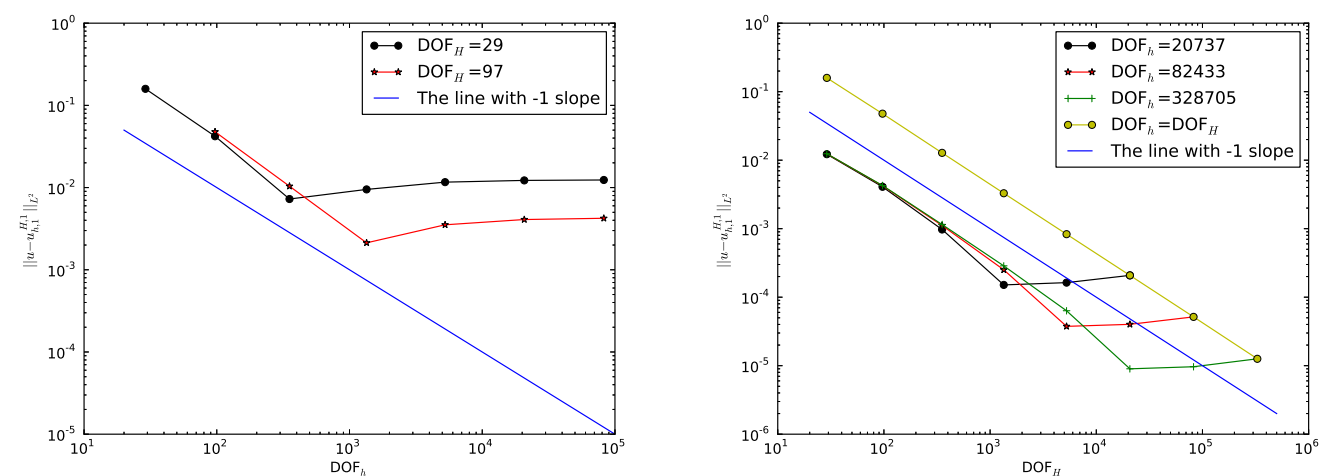

FIG. 6. Numerical errors $\left\|u-u_{h, 1}^{H, 1}\right\|_{L^{2}}\left(\mathbb{P}_{1}\right.$ finite elements), as functions of $\mathrm{DOF}_{h}$ (left) and $\mathrm{DOF}_{H}$ (right) (in $\log -\log$ scale).
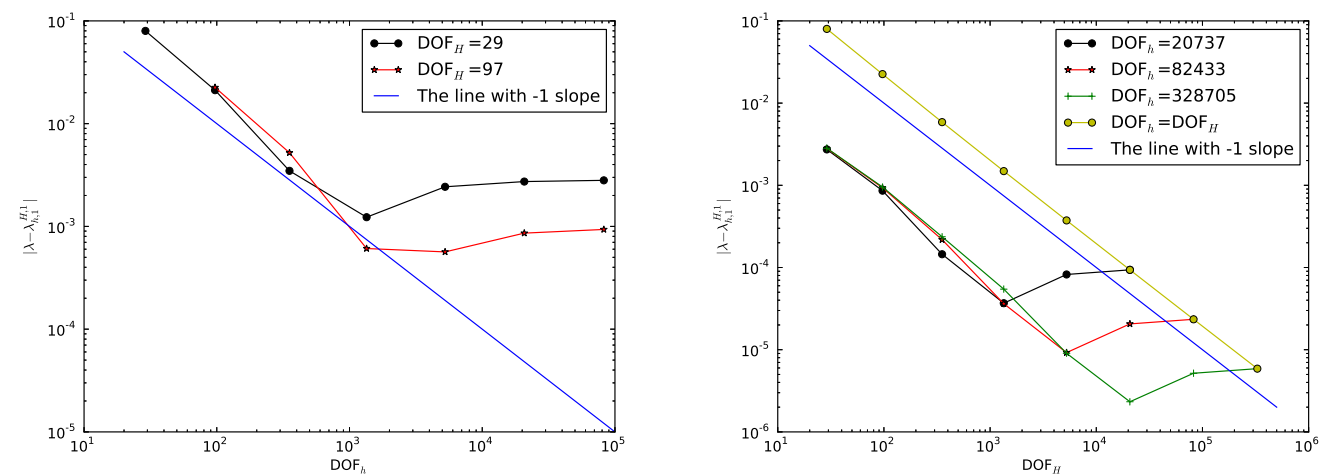

FIG. 7. Numerical errors $\left|\lambda-\lambda_{h, 1}^{H, 1}\right|\left(\mathbb{P}_{1}\right.$ finite elements), as functions of $\mathrm{DOF}_{h}$ (left) and $\mathrm{DOF}_{H}$ (right) (in log-log scale). 

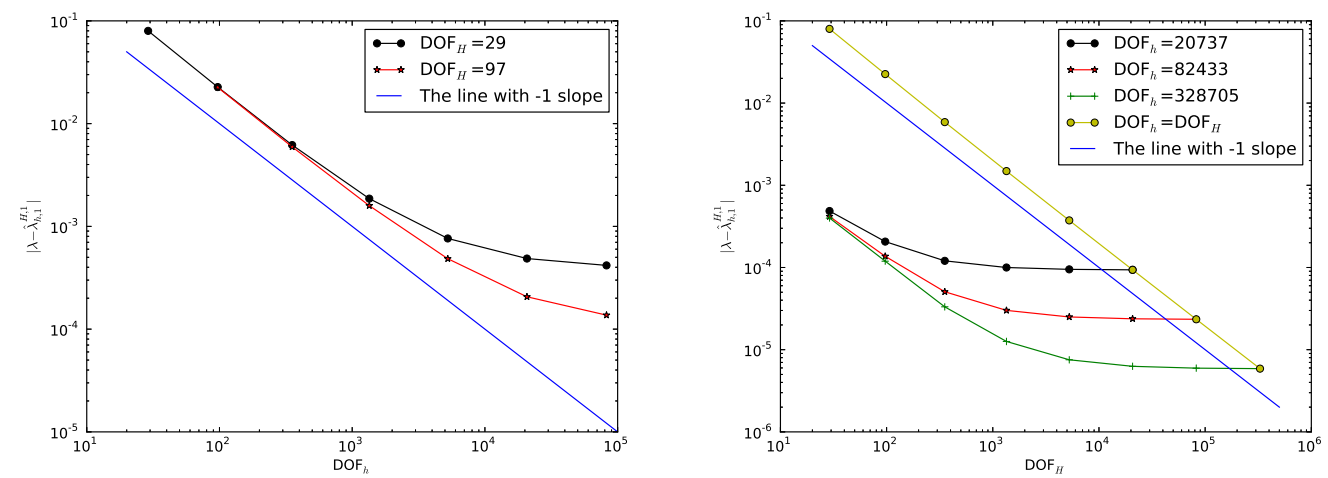

FIG. 8. Numerical errors $\left|\lambda-\tilde{\lambda}_{h, 1}^{H, 1}\right|\left(\mathbb{P}_{1}\right.$ finite elements), as functions of $\mathrm{DOF}_{h}$ (left) and $\mathrm{DOF}_{H}$ (right) (in $\log -\log$ scale).
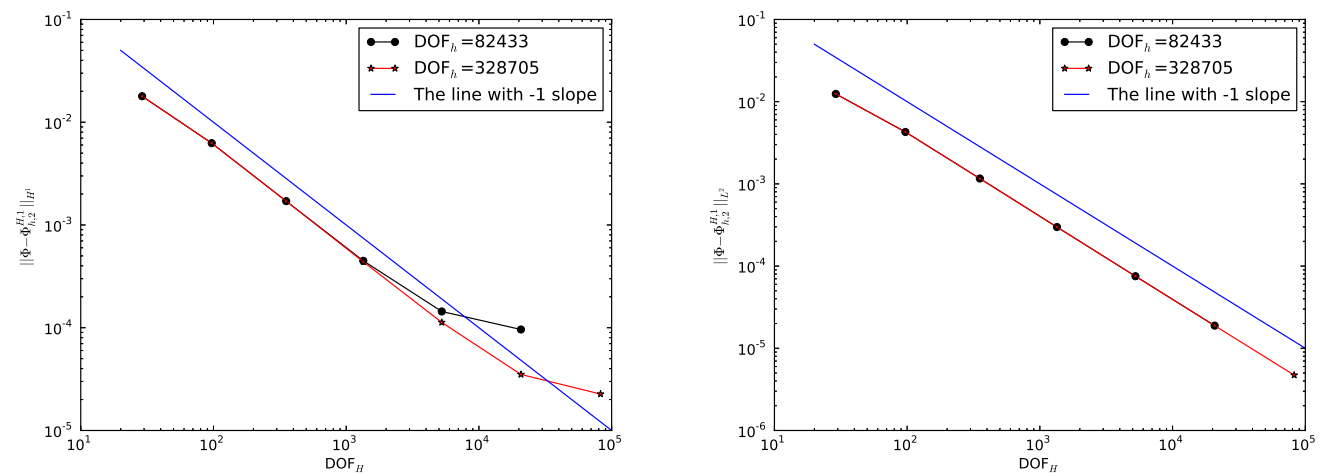

FIG. 9. Numerical errors $\left\|u-u_{h, 2}^{H, 1}\right\|_{H^{1}}$ (left) and $\left\|u-u_{h, 2}^{H, 1}\right\|_{L^{2}}$ (right), as functions of $\mathrm{DOF}_{H}$ (in log-log scale). 

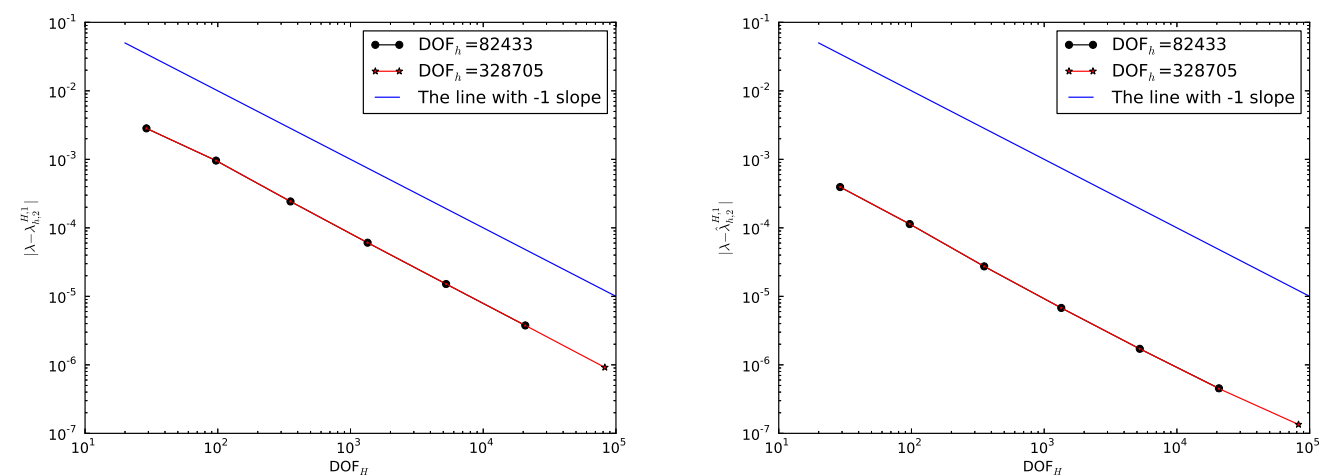

FIG. 10. Numerical errors $\left|\lambda-\lambda_{h, 2}^{H, 1}\right|$ (left) and $\left|\lambda-\tilde{\lambda}_{h, 2}^{H, 1}\right|$ (right), as functions of $\mathrm{DOF}_{H}$ (in log-log scale).
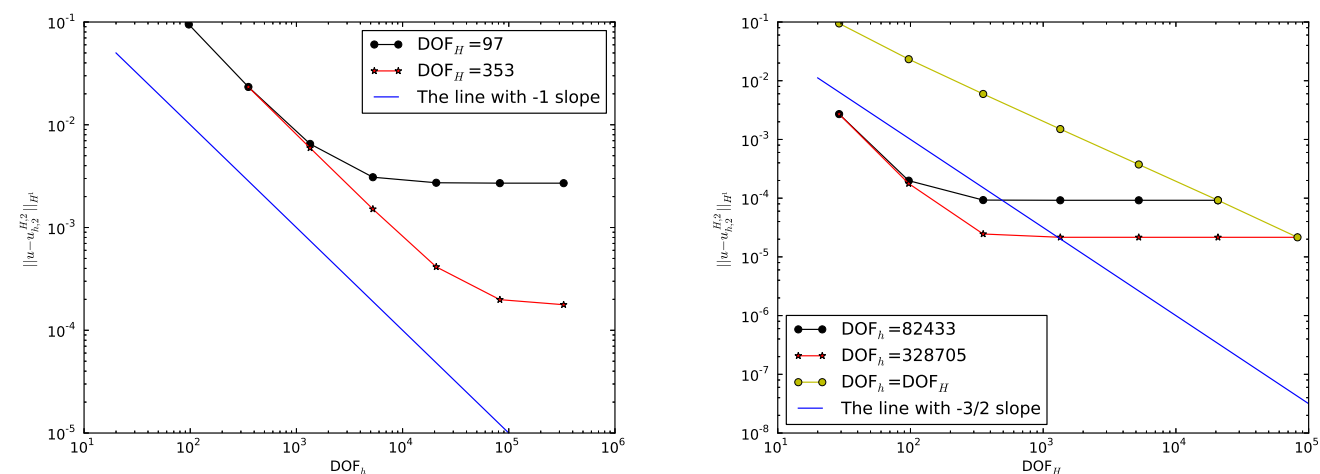

FIG. 11. Numerical errors $\left\|u-u_{h, 2}^{H, 2}\right\|_{H^{1}}$ ( $\mathbb{P}_{2}$ finite elements), as functions of $\mathrm{DOF}_{h}$ (left) and $\mathrm{DOF}_{H}$ (right) (in log-log scale).

We close this section with further finite element simulations on the nonlinear eigenvalue problem with varying potentials and nonlinearities. We address here only the eigenvalue approximation, because it is the only entity where the accuracy varies with these coefficients (the accuracy on the ground state energy or the ground state (eigenvector) are similar to what we presented previously). The problem is now : find $\left(u_{\delta_{F}}^{\delta_{C}}, \lambda_{\delta_{F}}^{\delta_{C}}\right) \in X_{\delta_{F}} \times \mathbb{R}$

$$
\int_{\Omega} \nabla u_{\delta_{F}}^{\delta_{C}} \nabla v_{\delta_{F}}+\int_{\Omega}\left(V+\theta u_{\delta_{C}}^{2}\right) u_{\delta_{F}}^{\delta_{C}} v_{\delta_{F}}=\lambda_{\delta_{F}}^{\delta_{C}} \int_{\Omega} u_{\delta_{F}}^{\delta_{C}} v_{\delta_{F}}, \quad \forall v_{\delta_{F}} \in X_{\delta_{F}}
$$



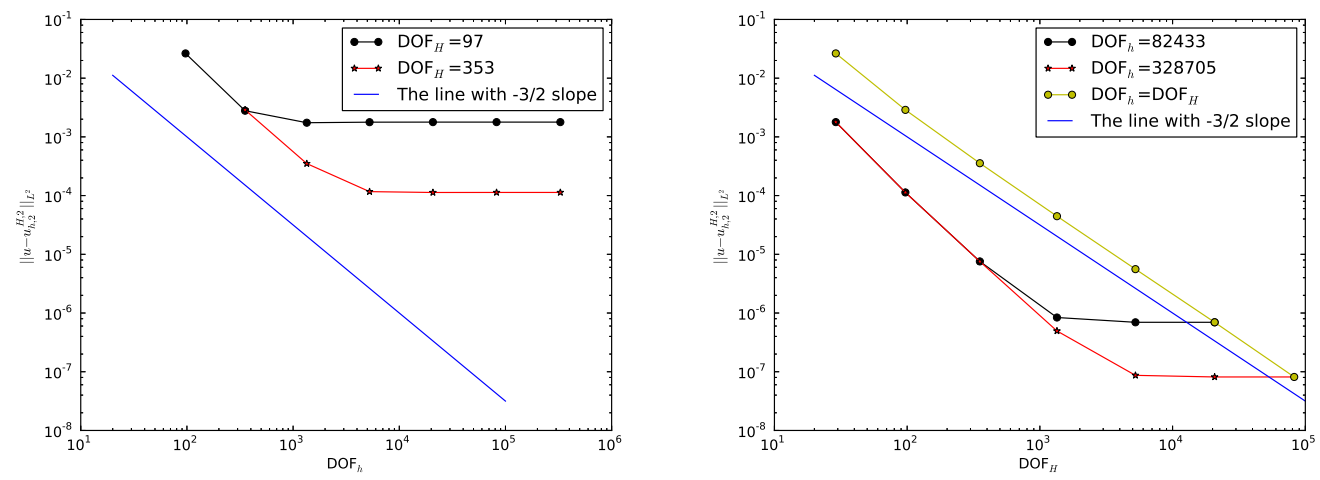

FIG. 12. Numerical errors $\left\|u-u_{h, 2}^{H, 2}\right\|_{L^{2}}$ ( $\mathbb{P}_{2}$ finite elements), as functions of $\mathrm{DOF}_{h}$ (left) and $\mathrm{DOF}_{H}$ (right) (in log-log scale).
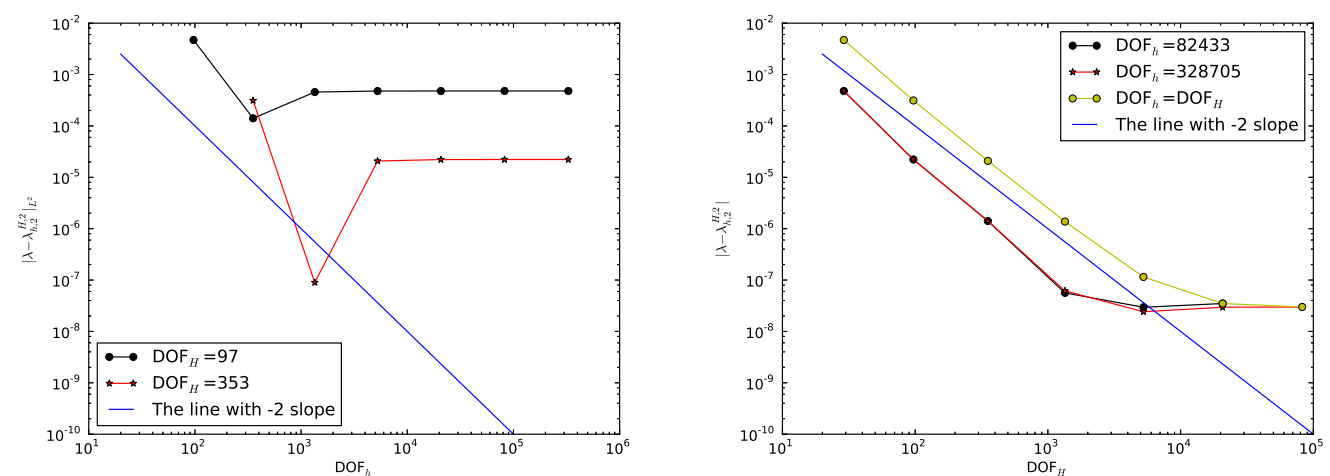

FIG. 13. Numerical errors $\left|\lambda-\lambda_{h, 2}^{H, 2}\right|\left(\mathbb{P}_{2}\right.$ finite elements), as functions of $\mathrm{DOF}_{h}$ (left) and $\mathrm{DOF}_{H}$ (right) (in log-log scale).
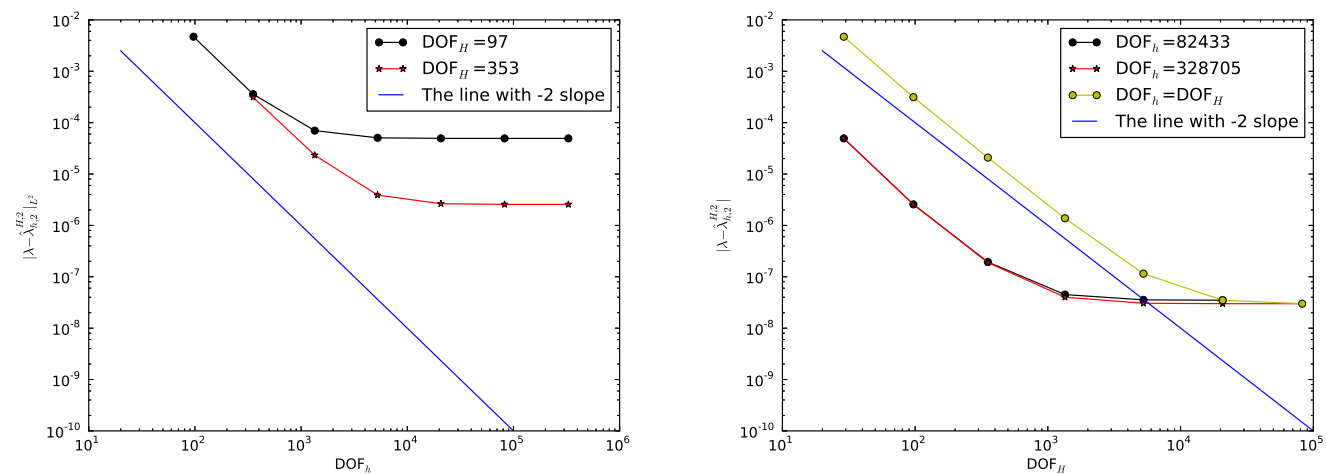

FIG. 14. Numerical errors $\left|\lambda-\tilde{\lambda}_{h, 2}^{H, 2}\right|\left(\mathbb{P}_{2}\right.$ finite elements), as functions of $\mathrm{DOF}_{h}$ (left) and $\mathrm{DOF}_{H}$ (right) (in $\log$-log scale). 

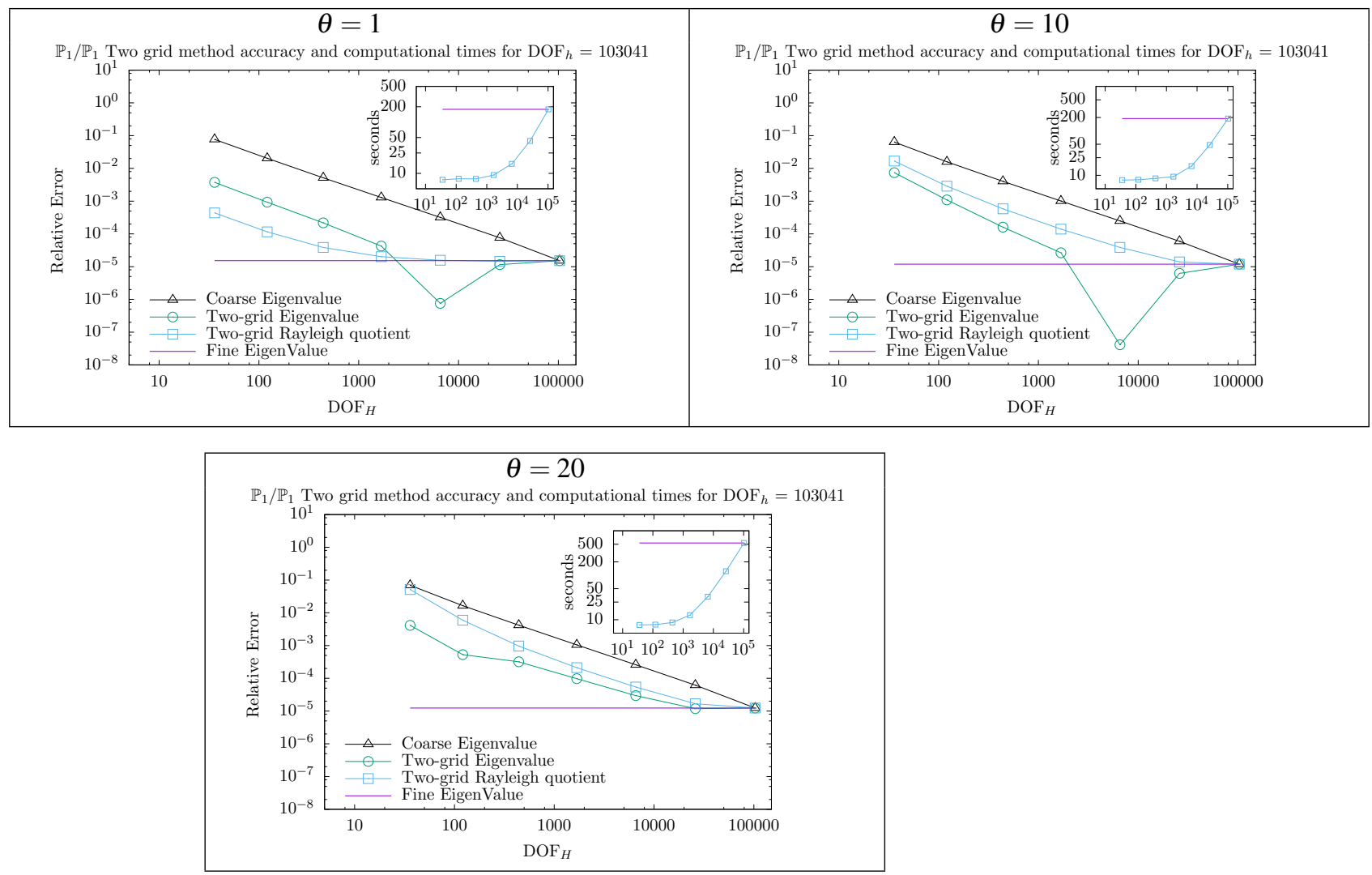

FIG. 15. Numerical errors $\left|\lambda-\lambda_{h, 1}^{H, 1}\right|\left(\mathbb{P}_{1}\right.$ finite elements), as functions of $\mathrm{DOF}_{H}$ for various values of $\theta$ for a regular potential $V$ (in the insert the associated time for the simulations in sec.).

$$
\tilde{\lambda}_{\delta_{F}}^{\delta_{C}}=\int_{\Omega}\left(\nabla u_{\delta_{F}}^{\delta_{C}}\right)^{2}+\int_{\Omega} V\left(u_{\delta_{F}}^{\delta_{C}}\right)^{2}+\theta \int_{\Omega}\left(u_{\delta_{F}}^{\delta_{C}}\right)^{4}
$$

We want to illustrate the two-grid eigenvalue accuracy as a function of the value of $\theta$ and $V$ (using $\mathbb{P}_{1}$ finite element for both the coarse and fine grid).

Fig. 15 shows numerical errors with $\Omega=] 0, \pi\left[\right.$ and $V(x, y)=x^{2}+y^{2}$.

Fig. 16 and Fig. 17 show numerical errors with $\Omega=] 0, \pi\left[\right.$ and $V(x, y)=\left\{\begin{array}{cc}V_{0} & \left.\text { on } \Omega_{0}=\right] \frac{\pi}{3}, \frac{2 \pi}{3}{ }^{2} \\ 0 & \text { on } \Omega \backslash \Omega_{0} .\end{array}\right.$

These results illustrate the fact that, when $\theta$ increases, the constant $C_{1}$ increases and the convergence rate of the two grid method gets closer to the coarse approximation. This effect is reduced for large values of $V$ that has the opposite effect on ratio between $C_{1}$ that depends on $\theta$ and $C_{2}$ that depends on $V$. 

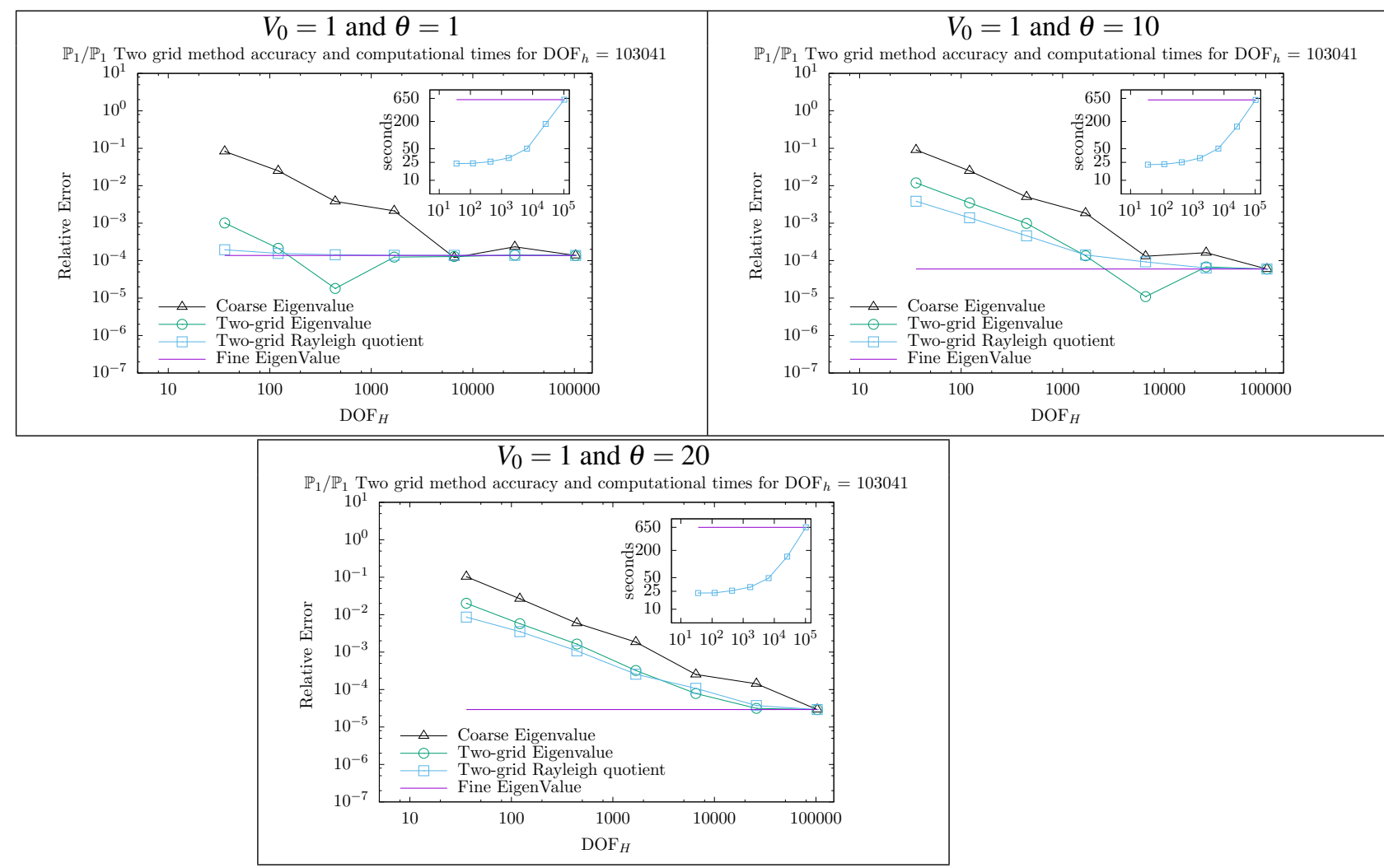

FIG. 16. Numerical errors $\left|\lambda-\lambda_{h, 1}^{H, 1}\right|\left(\mathbb{P}_{1}\right.$ finite elements), as functions of $\mathrm{DOF}_{H}$ for various values of $\theta$ for a mild discontinuous potential $V$ (in the insert the associated time for the simulations in sec.) . 


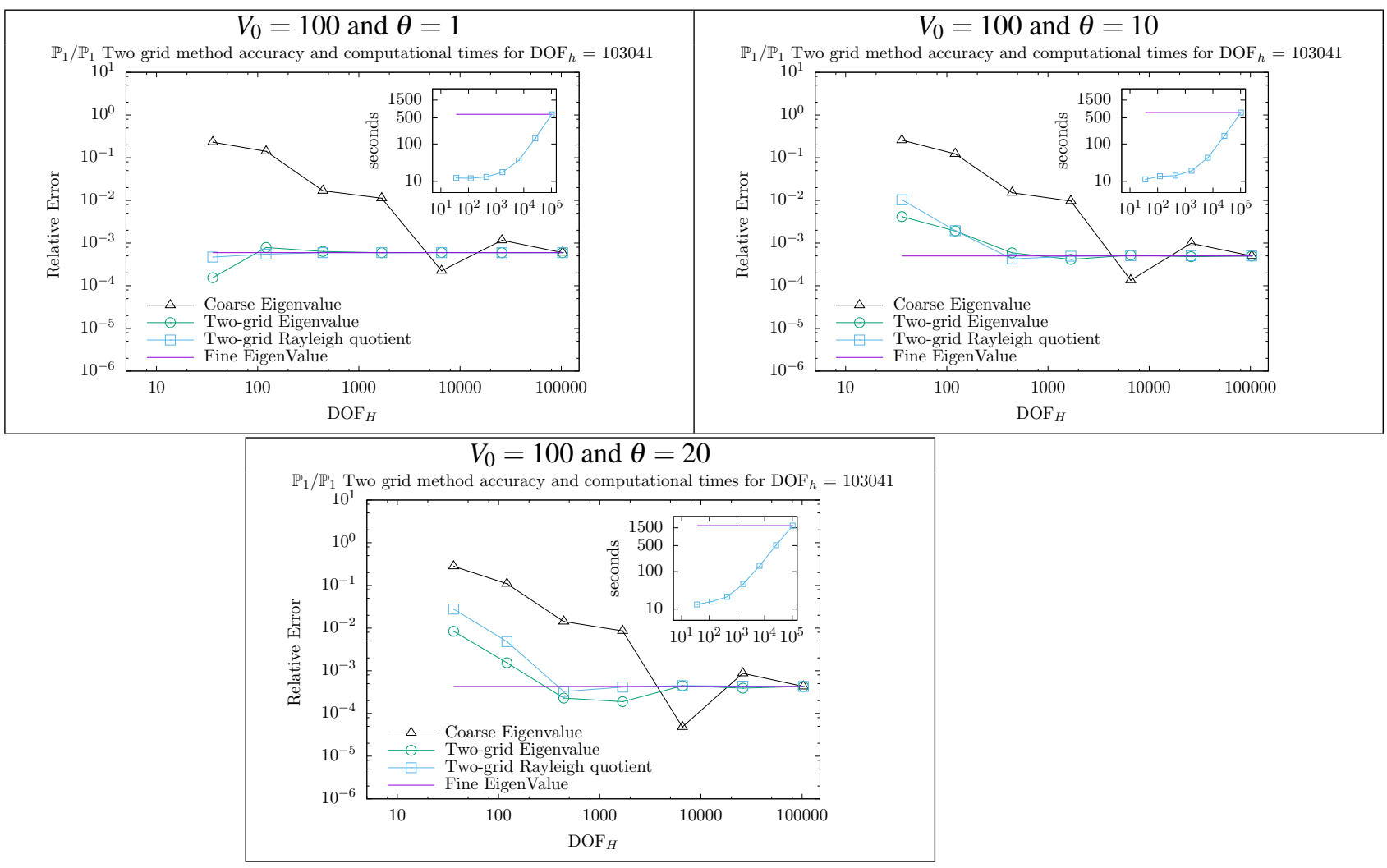

FIG. 17. Numerical errors $\left|\lambda-\lambda_{h, 1}^{H, 1}\right|\left(\mathbb{P}_{1}\right.$ finite elements), as functions of $\mathrm{DOF}_{H}$ for various values of $\beta$ for a strong discontinuous potential $V$ (in the insert the associated time for the simulations in sec.) . 
Finally, in this last set of numerical simulations we have indicated the time to solve the two grid eigenvalue problem and the time to solve the fine problem. These costs, that include all the operations, clearly reflect the reduced dimension of the nonlinear coarse approximation as we have checked that the number of (self-consistant field) iterations required to achieve convergence is independent on the size of the number of the degrees of freedom used for the discretization.

\section{Acknowledgements}

Financial support from the ANR grant Manif and from the French state funds managed by CALSIMLAB and the ANR within the Investissements d'Avenir programme under reference ANR-11-IDEX-0004-02, are gratefully acknowledged.

We are grateful to Ionut Danaila for his expertise on Gross-Pitaevskii equations and Bose-Einstein condensate.

We thanks the referees for their comments and questions that helped us to improve the final version of the paper.

\section{REFERENCES}

BAO, G. \& ZHOU, A. (2004) Analysis of finite dimensional approximations to a class of partial differential equations. Math. Methods Appl. Sci., 27, 2055-2066.

Bernardi, C., MadAy, Y. \& RAPETtTi, F. (2000) Discrétisations variationnelles de problèmes aux limites elliptiques. Springer.

Burke, K. (2012) Perspective on density functional theory. J. Chem. Phys., 136, 150901-150909.

CAncÈs E., Chakir, R. \& Maday, Y. (2010) Numerical analysis of nonlinear eigenvalue problems. J. Sci. Comput., 45, 90-117.

CAnCÈs E., ChakiR, R. \& MADAY, Y. (2012) Numerical analysis of the plane wave discretization of some orbital-free and Kohn-Sham models. Math. Model. Numer. Anal., 46, 341-388.

CHAKIR, R. (2009) Contribution à l'analyse numérique de quelques problèmes en chimie quantique et mécanique. $\mathrm{PhD}$ thesis, Université Pierre et Marie Curie.

Chen, H., Gong, X., He, L., YAng, Z. \& Zhou, A. (2013) Numerical analysis of finite dimensional approximations of Kohn-Sham models. Adv. Comput. Math., 38,. 225-256.

Ciarlet, P.G. \& Lions, J.-L. (1991) Handbook of numerical analysis, Vol.II: Finite element methods (Part I). North-Holland.

ERn, A. \& And Guermond J.-L. (2004) Theory and practice of finite elements. Springer.

Gilbarg, D. \& TRUdinger, N.S (1998) Elliptic partial differential equations of second order, 3rd edition, Springer

Hecht, F. (2012) New development in FreeFem++. J. Numer. Math., 20, 251-265.

Henning, P., MÅlqvist, A. \& Peterseim, D. (2014) Two-Level discretization techniques for ground state computations of Bose-Einstein condensates. SIAM Journal on Numerical Analysis, 52, 15-25

KoHN, W. (1999) Electronic structure of matter-wave functions and density functionals. Nobel Lecture.

KoHn, W. \& SHAM, L.J. (1965) Self-consistent equations including exchange and correlation effects. Phys. Rev., 140, A1133-A1138.

LAngwallner, B., ORTner, C. \& SÜLI, E. (2010) Existence and convergence results for the Galerkin approximation of an electronic density functional. Math. Mod. Meth. Appl. Sci., 20, 2237-2265.

PERdEW, J.P. \& ZUNGER, A. (1981) Self-interaction correction to density-functional approximations for manyelectron systems. Phy. Rev. B, 23, 5048-5079.

Pitaevskit, L.P. \& AND Stringari, S. (2003) Bose-Einstein Condensation. Clarendon, Oxford.

SICKEL, W. (1992) Superposition of functions in Sobolev spaces of fractional order. A survey. Banach Center 
Publ., 27, 481-497.

XU, J. \& ZHou, A. (2000) Local and parallel finite element algorithms based on two-grid discretizations. Math. Comput., 69, 881909.

ZHOU, A. (2004) An analysis of finite-dimensional approximations for the ground state solution of Bose-Einstein condensates. Nonlinearity, 17, 541-550. 